Publ. RIMS, Kyoto Univ.

14 (1978), 111-202

\title{
On the Theory of $b$-Functions
}

By

\author{
Tamaki YANO*
}

\section{CONTENTS}

Chapter I Generalities

$\S 1$. $\mathscr{D}[t, s]$-Modules and $b$-functions

$\S 2$. $b$-functions of holomorphic functions

Chapter II Structure of the ideal $g(s)$

$\S 3$. Total symbol

$\S$ 4. The numbers $L(f)$ and $l(f)$

$\S 5$. Functions of simplex type

\$ 6. Generators of $g(s)$

$\S 7$. "Fundamental conjecture" counter examples

$\S 8$. Generators of $\mathscr{G}(s)$-continued-

Chapter III Determination of $b(s)$

A. General Procedure

§ 9. Construction of eigenvectors

$\S 10$. Construction of operators

B. Isolated singularities

\$11. Quasi-homogeneous isolated singularities

§12. $L(f)=2$

$\S 13 . \quad L(f)=3$

$\S 14$. Examples of calculation

C. Local monodromy

$\S 15$. Relation with local monodromy structure

$\S 16$. Join formula for $b$-functions

Chapter IV Results of $b$-functions

$\S 17$. Two-dimensional case

§18. $x^{n_{1}}+y^{n_{2}}+x^{m} y^{m_{2}}$

$\S 19$. Other examples

$\S 20$. Remarks on the canonical forms of isolated singularities

$\S 21$. Non-isolated singularities

References

Communicated by M. Sato, March 14, 1977.

* Research Institute for Mathematical Sciences, Kyoto University, Kyoto 606, Japan. (Department of Mathematics, Kyoto University.)

Present address: Department of Mathematics, Saitama University, Urawa 338, Japan. 


\section{Notation}

$X:$ a complex manifold

$O_{X}$ : the sheaf of holomorphic functions on $X$

$\mathscr{D}_{X}$ : the sheaf of holomorphic linear differential operators of finite order on $X$

$\Theta_{X}$ : the sheaf of holomorphic vector fields on $X$

$\mathscr{D}_{X}[s]=\mathscr{D}_{X} \underset{\boldsymbol{C}}{\otimes} C[s]$

$\mathbb{N}$ : the set of natural numbers, $\mathbb{N}_{0}=\mathbb{N} \cup\{0\}$

$\mathbb{Z}$ : the set of rational integers

g.c.d. $=$ the greatest common divisor

1.c.m. $=$ the least common multiple

\section{Introduction}

The purpose of this paper is to develop a general theory of $b$-functions with emphasis on the detailed study of examples. A $b$-function $b_{f}(s)$ associated with a local holomorphic function $f(x)$ is defined to be a generator of the ideal formed by polynomials in $s$ satisfying

$$
P(s, x, D) f(x)^{s+1}=b(s) f(x)^{s},
$$

for some linear differential operator $P(s, x, D)=\sum_{0 \leq j \leq m} s^{j} P_{j}(x, D)$.

The following is a famous example of the equality of this type for a quadratic form $Q(x)=\sum_{i=1}^{n} x_{i}{ }^{2}$,

$$
\Delta Q^{s+1}=4(s+1)\left(s+\frac{n}{2}\right) Q^{s}
$$

where

$$
\Delta=\sum_{i=1}^{n} D_{i}{ }^{2}
$$

We note that the roots of $b_{f}(s)=0$ are strictly negative rational numbers.

It is well-known that $b$-function $b_{f}(s)$ plays important roles in analyzing hyperfunction $f_{ \pm}^{s}$. In fact, define the gamma factor $r(s)$ $=\Pi \Gamma\left(s+\alpha_{i}\right)$ when $b(s)=\Pi\left(s+\alpha_{i}\right)$. Then (1) turns out to be 


$$
P(s, x, D) \frac{1}{\gamma(s+1)} f^{s+1}=\frac{1}{\gamma(s)} f^{s} .
$$

In view of this formula, we can readily see that $f_{+}^{s}$ depends meromorphically on $s$ and its poles occur only at $-\alpha_{i}-\nu$, where $\nu$ runs over the non-negative integers.

For example, the factor $\left(s+\frac{n}{2}\right)$ in (2) has a relation with the following facts that (1) the poles of $Q_{+}^{s}$ are located at $-\frac{n}{2}-\nu, \nu \in \mathbb{N}_{0}$, (2) the local monodromy of $Q^{-1}(0)$ at 0 is $(-1)^{n}=\exp (2 \pi i(-n / 2))$, and (3) $I(h)=\int \exp ((i / h) Q(x)) \varphi(x) d x$ behaves asymptotically like $I(h)=0\left(h^{n / 2}\right),(h \rightarrow 0)$.

At this point, it should be remarked that $b_{f}(s)$ is an invariant of the hypersurface $f^{-1}(0)$ finer than local monodromy (cf. $\S 16$ etc.).

We investigate $b_{f}(s)$ through the structure of Modules $\eta=\mathscr{D}[s] f^{s}$ and $\mathscr{M}=\mathscr{D}[s] \int^{s} / \mathscr{D}[s] f^{s+1}$, where $\mathscr{D}[s]=\mathscr{D} \otimes \mathbb{C}[s]$. Here $s$ acts on them as $s: P(s) f^{s} \mapsto s P(s) f^{s}$. Then, one can state (1) as " $b_{f}(s)$ is the minimal polynomial of $s$ in $\mathcal{M}$." $\quad \mathcal{R}$ admits as pecial operation $t: P(s) f^{s}$ $1 \rightarrow P(s+1) f^{s+1}$. The commutation relation $t s-s t=t$ plays an essential role. This standpoint was established by M. Kashiwara and M. Sato.

This paper is organized as follows. In Chapter I, we introduce the notion of $\mathscr{D}[t, s]$-Modules, thereby $b$-functions being generally introduced. General theory of these Modules are included in [32]. In Chapter II, the structure of $\mathscr{N}$ and $\mathscr{M}$ are studied. The author introduces a number $L(f)$, which measures non-quasi-homogeneity of $f$, and the notion of a functions of simplex type. He also gives a good presentation of a Module $(s+1) \mathscr{M}$ for $f$ being isolated singularity with $L(f)=2,3$, which is used in the next chapter. The methods to determine or to estimate $b$-functions are investigated in Chapter III. The author gives a join-formula for $b$-functions in $\S 16$. Various examples are found in Chapter IV. In particular, some interesting explicit formulae are given for two-dimensional case. The determination of $b$-functions of all the canonical forms of isolated singularities with modality less than three is performed by the author and it is included in $\S 20$ for corank $f=2$ and in [32] for corank $f=3$. 
A part of the results of this work was announced in [27], [28], [29], [30], [34] and [37].

Historical remarks around the equation (1) is as follows.

I. M. Gelfand conjectured in Amsterdam Congress that the analytic properties of $f_{+}^{s}$ could be well investigated by use of the desingularization theorem. In fact, I. N. Bernstein-S. I. Gelfand [39] and M. F. Atiyah [38] proved the meromorphic dependence of $f_{+}^{s}$ in $s$ and discribed its poles by the resolution theorem of $\mathrm{H}$. Hironaka.

In 1961, M. Sato initiated a theory of $b$-functions for relative invariants on prehomogeneous vector spaces, in connection with the Fourier transforms and $\zeta$-functions associated with these spaces [23], [26].

On the other hand, I. N. Bernstein independently took the equation (1) and proved an existence theorem of such $b$-functions which does not ranish identically when $f$ is a polynomial [7]. J. E. Björk succeedingly generalized Bernstein's result for analytic functions [8].

Since then much effort has been forcused on the general theory of $b$-functions [12], [19], [27]. The author's contribution has been done since this stage. B. Malgrange pointed out a close connection between $b$-functions of $f$ and the local monodromy of $f^{-1}(0)$ [16]. He proved that, when $f$ has an isolated singularity, the eigenvalues of local monodromy are just $\exp (2 \pi \sqrt{-1} \alpha)$ for roots $\alpha$ of $b$-function [17]. Afterwards, M. Kashiwara proved the rationality of the roots of $b$-function for general $f$ in a completely different way [14].

As for the analytic property of $f^{\alpha}$, we note also that an important result that $f^{\alpha}$ satisfies a holonomic system was proved by I. N. Bernstein [7] in a special case and by M. Kashiwara-T. Kawai [10] for any $f$. More generally, analytic property of $f^{\alpha} u$ for holonomic $u$ is studied in [7] in a special case and in the author's subsequent paper [32] for general cases.

The $b$-functions associated with prehomogeneous vector spaces are well-investigated and they are determined by many people. The microlocal calculus finds its good application in the area of $b$-function theory, and that theme will be fully treated in M. Kashiwara-T. Kimura-M. Muro [41].

The author is grateful to Dr. M. Minami and Professor T. Kawai 
for their critical reading of the manuscripts. He would like to express his hearty gratitude to Professor M. Sato and Professor M. Kashiwara for their fruitful advices, enlightening discussions and constant encouragement.

\section{Chapter I. Generarities}

In this chapter, we study the basic features of general $\mathscr{D}[t, s]$ Modules and $b$-functions associated with them, which are indispensable to later chapters. The author develop the general theory of such $b$ functions and Modules in [32].

\section{$\S 1 . \mathscr{D}[t, s]$-Modules and $b$-Functions}

Let $\boldsymbol{C}[t, s]$ be the associative algebra over $\boldsymbol{C}$ with generators $s$ and $t$ and defining relation

$$
t s-s t=t .
$$

Set $\mathscr{D}[t, s]=\mathscr{D} \otimes C[t, s]$. A $\mathscr{D}$-Module $\mathscr{M}$ is called a $\mathscr{D}[s]$-Module (respectively $\mathscr{D}[t, s]$-Module), if $\mathscr{M} \supset s \mathscr{M}$ (respectively $\mathscr{M} \supset s \mathscr{M}$, $\mathscr{M} \supset t \mathscr{M})$ holds. In this chapter, all Modules are $\mathscr{D}[t, s]$-Modules unless otherwise stated. Since $t^{\nu} s=(s+\nu) t^{\nu}$ in view of (1), Ker $t^{\nu}$, Coker $t^{\nu}$ and $\operatorname{Im} t^{\nu}$ are $\mathscr{D}[t, s]$-Modules along with a given $\mathscr{D}[t, s]$. Module.

Definition 1. 1. Let $\mathcal{L}$ be a $\mathscr{D}[s]$-Module. If $s \in \mathcal{E n d}_{\mathscr{D}}(\mathcal{L})$ has the non- ero minimal polynomial, we denote it by $d_{\perp}(s)$, and say "d $d_{\perp}(s)$ exists." "b-functions" for a $\mathscr{D}[t, s]$-Module $\Omega$, are defined by $b_{\eta l, \nu}(s)=d_{\eta l / t v l l}(s), \nu=1,2 \cdots$.

Usually, $b_{\vartheta l, 1}$ is abbreviated as $b_{\eta l}$. As is easily seen, $b_{l, \nu}$ exist if and only if $b_{\mathfrak{n}}$ exists.

It should be remarked that if $\mathcal{L}$ is a holonomic $\mathscr{D}[t, s]$-Module $d_{\mathcal{L}}(s)$ exists, since $\mathcal{E n d}_{\mathscr{D}}(\mathcal{L})_{x}(x \in X)$ is finite dimensional and $\mathcal{E}_{\mathscr{D}}(\mathcal{L})$ is coherent [13].

Standard example of $\mathscr{D}[t, s]$-Module is constructed as follows. Let 
$f$ be a holomorphic function on $U \subset X$, let $\mathcal{L}$ be a coherent $\mathscr{D}$-Module and let $u$ be its section over $U$. We denote the annihilator of $u$ by $\mathcal{G}$, that is; $\mathscr{I}=\{Q \in \mathscr{D} \mid Q u=0\}$. Define the ideal $\mathscr{g}(s) \subset \mathscr{D}[s]$ by the condition that

$$
\begin{aligned}
& P(s, x, D) \in \mathcal{G}(s) \quad \text { if and only if } \\
& f^{m} P\left(s, x, D+\frac{s}{f} \operatorname{grad} f\right) \in \boldsymbol{C}[s] \otimes \mathcal{G}, \text { for some } m .
\end{aligned}
$$

We denote by $\Omega$ the Module $\mathscr{D}[s] / \mathscr{g}(s)$ and by $f^{s} u$ the class $(1 \bmod g(s)) . \quad \Re=\mathscr{D}[s] f^{s} u$ is a $\mathscr{D}[t, s]$-Module with actions of $t$ and $s$ given by,

$$
\iota: P(s) \mapsto P(s+1) f, \quad s: P(s) \mapsto P(s) s .
$$

The map $t$ is injective in $\Re$. In fact, if $P(s+1) f \in \mathcal{g}(s)$ then

$$
f^{m} P\left(s+1, x, D+\frac{s}{f} \operatorname{grad} f\right) f=\sum Q_{j} s^{j}
$$

for some $m$ and $Q_{j} \in \mathcal{G}$. The left-hand side equals to

$$
f^{m+1} P\left(s+1, x, D+\frac{s+1}{f} \operatorname{grad} f\right),
$$

and the right-hand side can be rewritten in the form

$$
\sum R_{j}(s+1)^{j}
$$

for some $R_{j} \in \mathcal{G}$. Therefore,

$$
f^{m+1} P\left(s, x, D+\frac{s}{f} \operatorname{grad} f\right)=\sum R_{f} s^{j},
$$

which implies $P(s) \in y(s)$.

The $\mathscr{D}$-Module $\mathscr{D} f^{s} u$ is coherent, and if $u$ is a holonomic section, D) $f^{s} u$ is subholonomic (see [32]).

Definition 1.2. With a non-zero polynomial $p(s)$, we associate a number $w(p) \in \mathbb{N}_{0}$ in the following manner $(w(p)$ is called the width of $p$.)

i) If $p(s) \in \mathbb{C}^{*}$ then $w(p)=0$,

ii) If $p(s)=\prod_{i=0}^{k}(s+\alpha+i)^{\varepsilon_{i}}, \alpha \in C, \varepsilon_{0} \varepsilon_{k} \neq 0$ then $w(p)=k+1$, 
iii) If $p(s)$ has the form

$$
\begin{aligned}
& p(s)=\prod_{i=1}^{k} p_{j}(s), \text { where each } p_{j}(s) \text { is of the form in ii), } \\
& p_{j}(s)=\prod\left(s+\alpha_{j}+i\right)^{\varepsilon_{i}(j)}, \text { and } \alpha_{j} \neq \alpha_{j^{\prime}} \bmod \mathbb{Z}\left(j \neq j^{\prime}\right) ; \text { then } \\
& w(p)=\max _{j} w\left(p_{j}\right) .
\end{aligned}
$$

Theorem 1.3. If $d_{\perp}(s)$ exists, then $t^{w\left(d_{\perp}\right)} \mathcal{L}=0$. Furthermore if we assume that $t$ is injective or surjective, then $\mathcal{L}=0$.

Proof. we have

$$
0=d_{\mathcal{L}}(s) \mathcal{L} \supset d_{\mathcal{L}}(s) t^{\omega\left(d_{\llcorner}\right)} \mathcal{L},
$$

and by virtue of (1),

$$
0=t^{w(d \mathcal{L})} d_{\mathcal{L}}(s) \mathcal{L}=d_{\mathcal{L}}\left(s+w\left(d_{\mathcal{L}}\right)\right) t^{w\left(d_{\perp}\right)} \mathcal{L} .
$$

It follows from the definition of $w\left(d_{\perp}\right)$ that

$$
\text { g.c.d. }\left(d_{\perp}(s), d_{\perp}\left(s+w\left(d_{\perp}\right)\right)=1\right. \text {. }
$$

Hence the assertion follows. When $t$ is injective or surjective, it is obvious that $\mathcal{L}=0$.

Q.E.D.

A coherent $\mathscr{D}$-Module $\mathcal{L}$ is called holonomic (resp. sub-holonomic) if $\mathcal{E}_{t_{\mathscr{D}}}^{i}(\mathcal{L}, \mathscr{D})=0$ for $i<n$ (resp. $\left.i<n-1\right)$. This condition is equivalent to $\operatorname{codim} \check{S S}(\mathcal{L}) \geq n$ (resp. $\operatorname{codim} \check{S S}(\mathcal{L}) \geq n-1$ ). $\mathcal{L}$ is called purely subholonomic if $\operatorname{Ext}_{\mathscr{D}}^{i}(\mathcal{L}, \mathscr{D})=0$ for $i \neq n-1$. It is known that for any coherent $\mathscr{D}$-Module, $\mathcal{E}_{\mathscr{D}} t_{\mathscr{D}}^{n}(\mathcal{L}, \mathscr{D})\left(\operatorname{resp} . \mathcal{E} x t_{\mathscr{D}}^{n-1}(\mathcal{L}, \mathscr{D})\right.$ ) is holonomic (resp. sub-holonomic) and $\mathcal{E}_{x t_{\mathscr{D}}^{i}}(\mathcal{L}, \mathscr{D})=0, i>n$. Let $W$ be an irreducible component of $\check{S} S(\mathcal{L})$. Then the multiplicity of $\mathcal{L}$ at a generic point $x_{0}$ of an irreducible component of $\breve{S S}(\mathcal{L})$ can be defined (which is denoted by $m_{x_{0}}(\mathcal{L})$ ), and has the additivity, that is, if

$$
0 \leftarrow \mathcal{L}_{1} \leftarrow \mathcal{L}_{2} \leftarrow \mathcal{L}_{3} \leftarrow 0,
$$

is an exact sequence of coherent $\mathscr{D}$-Modules, $m_{x_{0}}\left(\mathcal{L}_{2}\right)=m_{x_{0}}\left(\mathcal{L}_{1}\right)$ $+m_{x_{0}}\left(\mathcal{L}_{3}\right)$.

Corollary 1.4. Let $\Re$ be a sub-holonomic $\mathscr{D}[t, s]$-Module such that $t: \Omega \rightarrow \Re$ is injective. Then, $\Re$ is purely sub-holonomic. 
Proof. Consider the exact sequence

$$
0 \leftarrow \eta / t \eta \leftarrow \eta \stackrel{t}{\leftarrow} \eta \leftarrow 0
$$

Set $\mathcal{L}=\mathcal{E}_{x} t_{\mathscr{D}}^{n}(\mathcal{N}, \mathscr{D})$. Then $\mathcal{L}$ is holonomic and the long exact sequence of $\mathcal{E} x t$ gives us the surjection $\mathcal{L} \stackrel{t}{\rightarrow} \mathcal{L} \rightarrow 0$. Therefore $\mathcal{L}=0$ by virtue of Theorem 1.3. Q.E.D.

Proposition 1.5. Upon the conditions in Corollary 1. 4, $b_{\Re}$ exists.

Proof. Consider an irreducible component $W$ of $\check{S S}(\mathcal{S})$. Since $t$ is injective, the multiplicity of $\eta / t \eta$ at a generic point of $W$ vanishes. Therefore codim $\breve{S S}(\Re / t \Omega) \geq n$ which implies that $\Re / t \Re$ is holonomic. Thus $b_{\Re}$ exists (and so does $b_{\Re, \nu}$, by the argument after Definition 1. 1).

Q.E.D.

The conditions in Corollary 1.4 are satisfied for $\Omega=\mathscr{D}[s] f^{s} u$, if one of the following two conditions holds.

i) $f$ is arbitrary holomorphic function, $u=1$.

ii) $f$ is quasi-homogeneous, $\mathscr{D} u$ is holonomic.

In the present paper, we restrict ourselves to case i). We investigate case ii) in [32], where the detailed structure of $b_{\lambda, \nu}(s)$ and the relation between $\Re_{\alpha}$ and $\mathscr{D} f^{\alpha} u(\alpha \in C)$ are also discussed. The existence of $b_{\Re}(s)$ for $\Re=\mathscr{D}[s] f^{s} u$ with general $f$ and $\mathscr{D} u$ being holonomic can be derived from that of case ii), following the technique in $\S 3$ of [14]. (See [32])

\section{§ 2. b-Functions of Holomorphic Functions}

Let $X$ be a complex manifold of dimension $n$, and let $f(x)$ be a holomorphic function. Hereafter we make use of the notations $f_{i}=\partial f / \partial x_{i}$, $\mathfrak{a}=\sum \mathcal{O} f_{i}$, for brevity.

The $b$-function of $f$, which we denote by $b_{f}(s)$, is defined by,

$$
b_{f}(s)=b_{\Re l}(s) \text {, where } \quad \Re=\mathscr{D}[s] f^{s} .
$$

Here, $\boldsymbol{\Omega}$ is a special case of $\mathscr{D}[s] f^{s} u$ for $u=1$. We also define $b_{f, \nu}(s)=b_{9 l, \nu}(s)$. The existence of them will be assured later by Theorem 
1. 8.

It follows from the above definition that there are $P(s)$ and $P_{\nu}(s+\nu) \in \mathscr{D}[s]$, such that

$$
\begin{aligned}
& P(s) f^{s+1}=b_{f}(s) f^{s}, \\
& P_{\nu}(s+\nu) f^{s+\nu}=b_{f, \nu}(s) f^{s},
\end{aligned}
$$

and $b_{f}(s)$ and $b_{f, \nu}(s)$ are minimal among such polynomials in $s$.

When we emphasize the point $x \in X$ into consideration, we use the notation $b_{f, x}(s)$. Furthermore given a compact set $K \subset X$, we set $b_{f, K}(s)=\underset{x \in K}{1 . \operatorname{c.m}} . b_{f, x}(s)$.

If $f(x) \neq 0$, then $\frac{1}{f} f^{s+1}=f^{s}$. Hence $b_{f, x}(s)=1$.

If $f(x)=0$, setting $s=-1$ in (1), we know $(s+1) \mid b_{f, x}(s)$.

If $f(x)=0, \operatorname{grad} f(x) \neq 0$, then $\quad b_{f, x}(s)=(s+1)$ by $\frac{1}{f_{1}} D_{1} f^{s / 1}$ $=(s+1) f^{s}$ (e.g. when $\left.f_{1}(x) \neq 0\right)$.

Therefore, our main concern is with $b_{f, x}(s)$ at a singular point of $\int^{-1}(0)$.

If $y$ is in a sufficiently small neighborhood of $x, b_{f, y}(s) \mid b_{f, x}(s)$ by (1). For $g(x) \in \mathcal{O}, g\left(x_{0}\right) \neq 0$, we have $b_{g f, x_{0}}(s)=b_{f, x_{0}}(s)$. Because, if $P(s, x, D) f^{s+1}=b_{f}(s) f^{s}$,

$$
g^{-1} P(s, x, D-(s+1) \operatorname{grad} \log g)(g f)^{s+1}=b_{f}(s)(g f)^{s},
$$

and vice versa. Thus, $b_{f}(s)$ is an invariant of the hypersurface $\{f=0\}$ independent of the choice of its defining equation.

For later convenience we list up basic notations in $b$-function theory.

\section{Definition 1. 6.}

i) $\mathcal{J}(s)=\left\{P(s) \in \mathscr{D}[s] \mid P(s) f^{s}=0\right\}$,

$$
\begin{aligned}
& \mathcal{I}_{0}=\mathscr{D} \cap \mathcal{I}(s), \quad \mathcal{G}=\{X \in \Theta \mid X f \in \mathcal{U}\}, \\
& \mathscr{M}=\Re / t \Re, \quad \widetilde{M}=(s+1) \mathcal{M}, \quad \Omega_{a}=\Re /(s-\alpha) \Re .
\end{aligned}
$$

ii) $W=\{(x, s \operatorname{grad} \log f) \mid s \in C, f(x) \neq 0\}^{\text {closure }} \subset T^{*} X$, $W_{0}=\{(x, \xi) \in W \mid f(x)=0\} \cup\{(x, 0) \mid x \in X\}$.

Proposition 1. 7. $\Omega=\mathscr{D}[s] / \mathcal{I}(s)$, 


$$
\begin{aligned}
& \mathscr{M}=\mathscr{D}[s] /(\mathscr{g}(s)+\mathscr{D}[s] f) . \quad \widetilde{M}=\mathscr{D}[s] /(\mathscr{g}[s]+\mathscr{D}[s](\mathfrak{a}+\mathcal{O} f)), \\
& \Re_{\alpha}=\mathscr{D}[s] /(\mathscr{g}(s)+\mathscr{D}[s](s-\alpha))=\mathscr{D} /\left(\left.\mathscr{g}(s)\right|_{s=\alpha}\right) .
\end{aligned}
$$

Proof. The isomorphisms of $\mathcal{N}, \mathcal{M}$ and $\Re_{\alpha}$ are easy to verify. That of $\widetilde{\pi}$ is proved as follows. Let $P(s)$ be such that $P(s)(s+1) f^{s}$ $=Q(s) f^{s+1}$. Setting $s=-1$, we have $Q(-1)=\sum q_{i}(x, D) D_{i}$. Hence,

$$
\begin{aligned}
& P(s)(s+1) f^{s}=\left((s+1) R(s)+\sum q_{i}(x, D) D_{i}\right) f^{s+1} \\
& =(s+1)\left(R(s) f+\sum q_{i}(x, D) f_{i}\right) f^{s} . \\
& P(s) \in \mathscr{g}[s]+\mathscr{D}[s](\mathfrak{a}+\mathcal{O} f) \text {. }
\end{aligned}
$$

If $\operatorname{grad} f(x) \neq 0, f(x)=0$ at $x \in X$, we can assume $f=x_{1}$. Then $\mathscr{D}[s] f^{s}=\mathscr{D}[s] / \mathscr{D}[s]\left(s-x_{1} D_{1}\right)+\sum_{j=2}^{n} \mathscr{D}[s] D_{j} \simeq \mathscr{D} / \sum_{j=2}^{n} \mathscr{D} D_{j} . \quad$ Therefore, $\breve{S S}(\eta)=\left\{(x, \xi) \mid \xi_{2}=\cdots=\xi_{n}=0\right\}=W$ in a neighborhood of $x$. Since $\breve{S S}(\Re)$ is an analytic set, we have $\breve{S S}(\Re) \supset W$.

We state the fundamental theorem of M. Kashiwara.

Theorem 1.8. i) $\Re$ is sub-holonomic and $\breve{S S}(\Re)=W$. ii) $b_{f}(s)$ exists and all the roots of $b_{f}(s)=0$ are strictly negative rational.

For the proof of this, we refer the reader to M. Kashiwara [14]. The existence of $b_{f}(s)$ can be derived from i) and Proposition 1.5. See also [32].

Corollary 1.9. $\mathscr{M}, \widetilde{M}$ and $\Re_{\alpha}$ are holonomic. More precisely, $\breve{S S}(\mathscr{M}) \subset W \cap\left(f^{-1}(0)\right), \breve{S S}(\widetilde{\mathscr{M}}) \subset W \cap \operatorname{Sing} f^{-1}(0)$ and $\breve{S S}\left(\Re_{\alpha}\right) \subset W_{0}$.

Proof. For, $t$ gives an isomorphism on $W \backslash f^{-1}(0)$ in the exact sequence $0 \rightarrow \mathfrak{H} \stackrel{t}{\rightarrow} \mathscr{R} \rightarrow \mathscr{M} \rightarrow 0, \widetilde{S S}(\mathscr{M})$ is contained in $f^{-1}(0) \cap W$ and hence a holonomic set.

Since

$$
\begin{aligned}
& f D_{i}-f_{i} s \in g(s), \breve{S S}\left(\Re_{\alpha}\right) \subset W \cap\left(f^{-1}(0) \cup(\xi=0)\right) . \\
& \check{S S}(\mathscr{M}) \subset \breve{S S}(\mathcal{M}) \cap\left(f_{i}=0, \forall i\right) .
\end{aligned}
$$

When $f$ is locally reduced, K. Saito proved the following: 
Theorem 1. 10. $\mathcal{G}$ is a reflexive $\mathcal{O}_{X}$-Module. Let $X_{i}=\sum a_{i j}(x) D_{j}$ $i=1, \cdots, n$, be elements in $\mathcal{G}$. Then $X_{1}, \cdots, X_{n}$ is a locally free basis of $\mathcal{G}$, if and only if $\operatorname{det}\left(a_{i j}\right)=g f, g \in \mathcal{O}$.

Corollary 1.11. Suppose $\operatorname{dim} X=2$. Then $\mathcal{G}$ has locally free basis $X_{1}, X_{2}\left(X_{i}=\sum a_{i j} D_{j}\right)$ and $a_{11} a_{22}-a_{12} a_{21}=g f, g \in \mathcal{O}_{X}^{*}$. Converse$l y$, if two vector fields $X_{i}$ in $\mathcal{G}$ satisfy the above formula, they form a basis of $\mathcal{G}$.

For the proof of these, we refer the reader to K. Saito [21]. When $f$ is the square of the fundamental anti-invariant of a Coxeter group, considered as a function of fundamental invariants, $\mathcal{G}$ is a free module. This was pointed out by K. Saito [21]. For the determination of the structure of $\mathcal{G}$ and the microlocal structure of $\mathscr{D}[s] f^{s}$, we refer the reader to T. Yano [33] or T. Yano-J. Sekiguchi [35], [36]. They proved that the holonomic system $\mathscr{D} f^{\alpha}$ has multiplicity 1 on all the irreducible components of $\breve{S S}\left(\mathscr{D} f^{\alpha}\right)$, and determined a basis of $\mathscr{G}$ concretely.

Corollary 1.11 was also noted by M. Sato and M. Kashiwara (not published).

\section{Chapter II. Structure of the Ideal $g(s)$}

In this chapter, we shall restrict our attention to the structure of $g(s)$. First of all, we introduce a number $L(f)$, which measures the non-quasi-homogeneity of $f$. We further define a class of functions called a convergent power series of simplex type, which plays an important role in later applications. In the case of such a function, corresponding $g(s)$ contains a distinguished element (cf. Theorem 2.15). In $\S \S 6,8$, we shall determine the structure of $y(s)$ upon the following two conditions that $1^{\circ} L(f) \leq 3$ and $2^{\circ}$ the singularity is isolated. Section 8 is concerned with a delicate phenomenon about $q(s)$, and given are counter examples against Sato-Kashiwara conjectures.

\section{$\S 3$. Total Symbol}


For the later purposes it is appropriate to modify the notion of order of an element of $\mathscr{D}[s]$ by regarding $s$ as element of order 1 . To be more precise, we define

Definition 2. 1. Given $P(s)=\sum s^{j} P_{j}(x, D) \in \mathscr{D}[s]$, max $\left(j+\operatorname{ord} P_{j}\right)$ is called the total order of $P$ and denoted by $\operatorname{ord}^{T}(P(s))$. Let $l=\operatorname{ord}^{T}(P(s))$. Then we call

$$
\sigma^{T}(P)(s, x, \xi)=\sum s^{j} \sigma_{l-j}\left(P_{j}\right),
$$

the total symbol of $P$. It follows that $\sigma^{T}(P)$ is a function on $C \times T^{*} X$ having homogeneous degree $l$ in $(s, \xi)$. For an ideal $\mathcal{I}(s)$ in $\mathscr{D}[s]$, re define its total symbol ideal by

$$
\sigma^{T}(\mathcal{G}(s))=\left\{\sigma^{T}(P) \mid P \in \mathcal{G}(s)\right\} .
$$

Let $\mathcal{G}$ be an ideal in $\mathcal{O}_{T^{*} X}[s]$ and $S$ be a subset of $C \times T^{*} X$. Then we denote by $V(\mathcal{G})$ and $\mathcal{G}(S)$ the null set of $\mathcal{G}$ and the ideal of functions that vanish on $S$, respectively.

Definition 2.2. i) We define

$$
\breve{S S}[s](\mathcal{L})=V\left(\sigma^{T}(\mathcal{I}(s))\right) \text {, }
$$

for a $\mathscr{D}[s]$-Module $\mathcal{L}=\mathscr{D}[s] / \mathcal{I}(s)$. More generally we define

$$
\widetilde{S S}[s](\mathcal{L})=\bigcup_{i=1}^{l} \breve{S} S[s]\left(\mathscr{D}[s] u_{i}\right) \text {, }
$$

for finitely generated $\mathscr{D}[s]-$ Module $\mathcal{L}=\mathscr{D}[s] u_{1}+\cdots+\mathscr{D}[s] u_{l}$. ii) Let $f$ be a holomorphic function. The subset $W[s]$ in $C \times T^{*} X$ is defined by

$$
W[s]=\{(s, x, s \operatorname{grad} \log f) \mid f \neq 0, s \in C\}^{\text {closure }}
$$

Proposition 2.3.

$$
\breve{S S}[s](\Re)=W[s]
$$

Proof. Let $P(s) \in \mathscr{D}[s], \operatorname{ord}^{T} P=m$. Then $P(s) f^{s}=(s)_{m} \sigma^{T}(P)(f, x, d f) f^{s-m}+($ lower order in $s)$. 
Therefore, if $P(s) \ni \mathscr{g}(s)$, then $\sigma^{T}(P) \in \mathcal{I}(W[s])$. Let $p(s, x, \xi)$ $\in \mathcal{G}(W[s])$. We shall prove that $\exists l, \exists P(s) \in \mathcal{G}(s)$ such that $\sigma^{T}(P(s))$ $=p(s, x, \xi)^{l}$. From this, 2.3 follows. Define the function on $\boldsymbol{C} \times X$ $\ni(t, x)$ by $f^{\prime}(t, x)=t f(x)$, and the function on $T^{*}(\boldsymbol{C} \times X)$ by $q(t, x, \tau, \xi)$ $=p(t \tau, x, \xi)$. Since $p(s, x, s d \log f)=0$, we have $q\left(t, x, \frac{s}{t}, s d \log f\right)$ $=0$. Howerer, $\left(\frac{s}{t}, s d \log f\right)=s d_{(t, x)} \log f^{\prime}$. Hence, $q$ ranishes on $I^{\prime}=\left\{\left(t, x, s d_{(t, x)} \log f^{\prime}\right) \mid t f \neq 0, s \in C\right\}^{\text {closure }}$. By Theorem 1. 8, we have $\breve{S S}\left(\eta^{\prime}\right)=W^{\prime}$, where $\Re^{\prime}=\mathscr{D}_{\boldsymbol{C} \times X}[s] f^{\prime s}=\mathscr{D}_{\boldsymbol{C} \times X} f^{\prime s}$, whence there are $l \in \boldsymbol{N}$ and $Q \in g_{f^{\prime}}$ such that $\sigma(Q)=q^{l}$. We write $Q_{0}$ for the 0 -th homogeneous part of $Q$ with respect to $t$. Then obviously it follows that

$$
Q_{0}\left(t D_{t}, x, D_{x}\right) f^{\prime s}=0
$$

and $\sigma\left(Q_{0}\right)=q^{l}$. Finally define $P\left(s, x, D_{x}\right)=Q_{0}\left(s, x, D_{x}\right)$. Then, we readily have $P\left(s, x, D_{x}\right) f^{s}=0$ and $\sigma^{T}(P)=p^{l}$.

Q.E.D.

The above Proposition 2.3 amounts to saying that $\sigma^{T}(g(s))$ $\subset \mathcal{G}(W[s])$. There are examples of $f$ for which this inclusion relation are a strict one (cf. $\S 8$ ). A necessary condition for $p$ to belong to $\sigma^{T}(g(s))$ will be given in the following ( $1^{\circ}, 2^{\circ}, 3^{\circ}$ below).

Let $p(s, x, \xi) \in \mathcal{O}_{T^{*} X}[s]$ be a homogeneous function of degree $m$ in $(s, \hat{\xi}): p(s, x, \hat{\xi})=\sum_{|a|=j} s^{m-j} a_{j, \alpha} \xi^{\alpha}$. In the sequel, we use the notation

$$
\begin{aligned}
& R_{k}[p](s, x, \xi)=\sum_{|\alpha|=k+1} \frac{1}{\alpha !}\left(D_{x}^{\alpha} f\right)(x) \cdot\left(D_{\xi}^{\alpha} p\right)(s, x, \xi) \\
& R_{0}[p](s, x, \xi)=p(s, x, \xi)
\end{aligned}
$$

and

$$
p(s, x, D)=\sum_{j}(s-j)_{m-j} \sum_{|\alpha|=j} a_{j, \alpha} D^{\alpha} .
$$

Let $P(s)$ be an element in $\mathscr{D}[s]$ with $\operatorname{ord}^{T}(P(s))=m$. Then,

$$
P(s) f^{s}=a(s, x) f^{s-m},
$$

where $a(s, x)$ is a polynomial of order not greater than $m$ in $s$ with coefficients in $\mathcal{O}$. We use the notation

(order less than $m+1$ in $s$ ) 
for $a(s, x) f^{s-m}$. Let $a^{\prime}(s, x)$ be a polynomial of order less than $m$ in $s$ with coefficients in $\mathcal{O}$. Then the formula of the form

$$
a(s, x) f^{s-m}+a^{\prime}(s, x) f^{s-m}
$$

is denoted by

$$
a(s, x) f^{s-m}+(\text { lower order in } s) \text {. }
$$

First concider a homogeneous function $p(x, \xi)=\sum_{|\alpha|=k} a_{\alpha} \xi^{\alpha}$ not depending on $s$. Then we have

$$
\begin{aligned}
p(x, D) f^{s}= & (s)_{k} p(x, d f) f^{s-k}+\sum_{h=1}^{3}(s)_{k-h} R_{h}[p](x, d f) f^{s-k+h} \\
& +(\text { lower order in } s) .
\end{aligned}
$$

From this, we have for $p(s, x, \xi)=\sum s^{m-k} p_{k}(x, \xi)$ with the degree of $p_{k}$ in $\xi$ is just $k$,

$$
\begin{aligned}
p(s, x, D) f^{s}= & (s)_{m}\left(\sum f^{m-k} p_{k}(x, d f)\right) f^{s-m} \\
& +\sum_{h=1}^{3} \sum_{k}(s)_{k-h}(s-k)_{m-k} R_{h}\left[p_{k}\right] f^{s-k+h} \\
& +(\text { order less than } m-3 \text { in } s) \\
= & (s)_{m} p(f, x, d f) f^{s-m} \\
& +(s)_{m-1} R_{1}[p](f, x, d f) f^{s-m+1} \\
& -(s)_{m-2} R_{1}\left[\frac{\partial p}{\partial s}\right](f, x, d f) f^{s-m+2} \\
& +(s)_{m-3} R_{1}\left[\frac{\partial^{2} p}{\partial s^{2}}\right](f, x, d f) f^{s-m+3} \\
& +(s)_{m-2} R_{2}[p](f, x, d f) f^{s-m+2} \\
& -2(s)_{m-3} R_{2}\left[\frac{\partial p}{\partial s}\right](f, x, d f) f^{s-m+3} \\
& +(s)_{m-3} R_{3}[p](f, x, d f) f^{s-m+3} \\
& +(\text { order } \operatorname{less} \text { than } m-3)
\end{aligned}
$$

Now assume conditions:

$1^{\circ} p(f, x, d f)=0$, 
$2^{\circ} \quad R_{1}[p](f, x, d f) \in(\mathfrak{a}+\mathcal{O} f)^{m-1}$.

With the aid of the condition $2^{\circ}$, there is a homogeneous polynomial $p^{\prime}$ of degree $m-1$ such that

$$
p^{\prime}(f, x, d f)=R_{1}[p](f, x, d f) .
$$

Set

$$
P(s)=p(s, x, D)-p^{\prime}(s, x, D) .
$$

Then

$$
\begin{aligned}
P(s) f^{s}= & (s)_{m-2}\left(R_{2}[p]-R_{1}\left[\frac{\partial p}{\partial s}\right]-R_{1}\left[p^{\prime}\right]\right)(f, x, d f) \\
& +(\text { order less than } m-2)
\end{aligned}
$$

Thus we have the following theorem.

We define ideals $c_{l} l=2,3, \cdots$ by

$\mathfrak{c}_{l}=\left\{R_{1}[q](f, x, d f) \mid\right.$ hog. $\left.\operatorname{dem}_{(s, \xi)} q(s, x, \xi)=l, q(f, x, d f)=0\right\}$.

Note that

$$
c_{l} \subset(\mathfrak{a}+\mathcal{O} f)^{1-2}\left(\Sigma O f_{i j}\right)
$$

Theorem 2.4. Let $p(s, x, \xi) \in \mathcal{O}_{T+x}[s]$ be a homogeneous polynomial of degree $m$ in $(s, \xi)$. Then, to impose the condition $1^{\circ}$ and $2^{\circ}$ is equivalent to ensure the existence of an operator $P(s)$ which has the following propertics.

$$
\begin{aligned}
& \sigma^{T}(P(s))=p, \\
& P(s) f^{s}=(\text { order less than } m-1 \text { in } s) .
\end{aligned}
$$

Assume $1^{\circ}$ and $2^{\circ}$, and set

$$
R(x)=\left(R_{2}[p]-R_{1}\left[\frac{\partial p}{\partial s}\right]-R_{1}\left[p^{\prime}\right]\right)(f, x, d f),
$$

with $p^{\prime}$ iniroduced just before this theorem. Then there exists $P^{\prime}(s)$ such that

$$
\begin{aligned}
& \sigma^{r}\left(P^{\prime}(s)\right)=p \\
& P^{\prime}(s) f^{s}=(\text { order less than } m-2 \text { in } s)
\end{aligned}
$$


if and only if the following condition $3^{\circ}$ holds.

$3^{\circ} \quad R(x) \in(\mathfrak{a}+\mathcal{O} f)^{m-2}+\mathfrak{c}_{m-1}$.

Corollary 2.5. 1) If $P(s), \operatorname{ord}^{T} P=m$, satisfies

$1 \quad \sigma^{T}(P(s)) \in \sigma^{T}(g(s))$,

$2 P(s) f^{s}=s^{m-1} Q(x) f^{s-m+1}+($ lower order in $s)$,

then $Q(x) \in(\mathfrak{a}+\mathcal{O} f)^{m-1}$.

2) When $p_{2}(s, x, \tilde{\xi})=a s^{2}+\left(\sum a_{i} \xi_{i}\right) s+\sum a_{i j} \xi_{i} \xi_{j}$ satisfies $p_{2}(f, x, d f)$ $=0$, there exists $P(s) \in g(s)$ such that $\sigma^{T}(P)=p_{2}$ if and only if

$$
\sum a_{i j} f_{i j} \in \mathfrak{a}+\mathcal{O f} \text {. }
$$

\section{$\S$ 4. The Numbers $L(f)$ and $l(f)$}

Let $f \in \mathcal{O}_{X}$ such that $V(\mathfrak{a}) \subset V(f)$. We denote by $l(f)$ the degree of integral dependence of $f$ over $\mathfrak{a}$, whose existence is assured by the presence of

Theorem 2.6 (H. Hironaka). $f$ is integral over a.*)

Corollary 2. 7. There exists $P(s) \in \sum_{j=0}^{l} s^{l-j} P_{j}(x, D)$ in $g(s)$ such that $\operatorname{ord}^{T} P=l, P_{0}(x, D)=1$.

Proof. The $p(s, x, \xi)$ in Theorem 2.6 belongs to $\mathcal{I}(W[s])$. It follows then from Proposition 2.3 that there exist $k$ and $P(s) \in \mathcal{g}(s)$ satisfying

$$
\sigma^{T}(P(s))=p^{k}
$$

Obviously, this $P(s)$ is an anounced one.

Q.E.D.

We write $L(f)$ for the minimum of $\operatorname{ord}^{T} P$ where $P(s) \in \mathcal{G}(s)$ which is of the form specified in Corollary 2.7. We have $L(f)=1$ when and only when $f$ is quasi-homogeneous.

*) That is, there exists $p(s, x, \xi) \in \mathcal{O}_{X}[s, \xi]$, homogeneous in $(s, \xi)$ and $p(f, x, d f)=0$, $p(s, 0,0)=s^{\text {deg } p}$. 
Proposition 2.9 (K. Saito [22]). When $f$ has an isolated singularity, the condition $L(f) \geq 2$ is equivalent to the condition

$$
\operatorname{det}\left(\frac{\partial^{2} f}{\partial x_{i} \partial x_{j}}\right) \in \mathfrak{a}+\mathcal{O f}
$$

\section{$\S 5$. Functions of Simplex Type}

Let $f(x)$ be a local holomorphic function at $0 \in \mathbb{C}^{n}, f(0)=0$. We fix a local coördinate and expand $f$ into convergent power series. The support of $f$ in this coördinate is defined to be

$$
\operatorname{supp}(f)=\left\{\alpha \in \mathbb{N}_{0}^{n} \mid a_{\alpha} \neq 0 \text { in } f=\sum a_{\alpha} x^{\alpha}\right\} .
$$

The set of subsets $S$ of $\operatorname{supp}(f)$, satisfying $S+\mathbb{N}_{0}^{n} \supset \operatorname{supp}(f)$ has the minimal element, which is denoted by $\operatorname{inl}(f)$. This set can alternatively be described in the following way. Define the order relation < on $\mathbb{N}_{0}^{n}$ by

$$
\alpha<\alpha^{\prime} \text { if and only if } \alpha_{i} \leq \alpha_{i}^{\prime}, \forall i
$$

Then $\operatorname{inl}(f)$ is characterized by

$$
1^{\circ} \forall \beta \in \operatorname{supp}(f),{ }^{\exists} \alpha \in \operatorname{inl}(f) \text { such that } \alpha<\beta \text {, }
$$

and

$2^{\circ} \quad \forall \alpha, \alpha^{\prime}\left(\alpha \neq \alpha^{\prime}\right) \in \operatorname{inl}(f)$, there is no relation $\alpha<\alpha^{\prime}$.

Thus we can write $f(x)=\sum_{\alpha \in \operatorname{in} l(f)} a_{\alpha}(x) x^{\alpha}, a_{\alpha}(0) \neq 0$, and for any $x^{\alpha}$, $x^{\alpha^{\prime}}, \alpha, \alpha^{\prime} \in \operatorname{inl}(f), x^{\alpha}$ is not a divisor of $x^{\alpha^{\prime}}$.

Incidentally, the following proposition holds.

Proposition 2. 10. If $\operatorname{inl}(f)=\left\{\alpha^{(1)}, \cdots, \alpha^{(n)}\right\}$ forms a set of vertices of $(n-1)$-simplex, $f$ can be transformed to $\sum y^{a^{(i)}}$ by an appropriate coördinate change $(x) \mapsto(y)$.

Proof. Let $A$ be the matrix $\left(\begin{array}{c}\alpha^{(1)} \\ \vdots \\ \alpha^{(n)}\end{array}\right)$. Then it follows from the condition that $A$ is invertible. Set $\left(\begin{array}{c}b_{1} \\ \vdots \\ b_{n}\end{array}\right)=A^{-1}\left(\begin{array}{cc}\log & a_{1} \\ \vdots \\ \log & a_{n}\end{array}\right)$, where $a_{i}$ 's are 
$f=\sum a_{i}(x) x^{\alpha^{(i)}}$, and set $y_{i}=\exp \left(b_{i}\right) x_{i}$. Then $f(x(y))=\sum_{i=1}^{n} y^{\alpha^{(i)}}$.

Q.E.D.

In this case, $f$ can be considered to be weighted homogeneous with weight

$$
\left(\gamma_{1}, \cdots, \gamma_{n}\right)=(1, \cdots, 1)^{t} A^{-1}
$$

Definition 2. 11. When $\operatorname{inl}(f)=\left(\alpha^{(0)}, \alpha^{(1)}, \cdots, \alpha^{(n)}\right)$ forms a set of vertices of n-simplex, we call that $f$ is of simplex type.

In the sequel, we fix some coördinate system, and use the phraze, "of simplex type" to mean "of simplex type in that coördinate".

Proposition 2.12. Let $f$ be of simplex type. Then, by an appropriate change of coördinate $(x) \rightarrow(y)$, there is found a function $g(y), g(0) \neq 0$, such that

$$
f(x(y))=g(y) \sum_{i=0}^{n} y^{\alpha^{(i)}} .
$$

Proof. First note that $A^{\prime}=\left(\begin{array}{cc}\alpha^{(0)} & 1 \\ \vdots & \vdots \\ \alpha^{(n)} & 1\end{array}\right)$ is invertible in this case. In fact, if it were not so, $\left\{\alpha^{(0)}, \cdots, \alpha^{(n)}\right\}$ should lie in some hyperplane. We set $\left(\begin{array}{c}b_{1} \\ \vdots \\ b_{n} \\ b\end{array}\right)=A^{\prime-1}\left(\begin{array}{c}\log a_{0} \\ \vdots \\ \log a_{n-1} \\ \log a_{n}\end{array}\right), y_{i}=\exp \left(b_{i}\right) x_{i}$ and $g(y)=\exp (b)$. Then $f(x(y))=g(y) \sum_{i=0}^{i} y^{\alpha^{(i)}}$.

It is thus found that the $b$-function of $f$ at 0 is equal to that of $\sum_{i=0}^{n} c_{i} x^{a^{(i)}}$ for any $c_{i} \in \boldsymbol{C}^{*}$.

Theorem 2. 13. Let $\alpha_{0}, \cdots, \alpha_{n} \in \boldsymbol{N}_{0}^{n} \backslash(0)$ form a set of vertices of an n-simplex. Then, by an appropriate change of subscripts, if necessary, one can determine $I_{j} j=1,2,3$ uniquely which satisfy the following: 


$$
I_{1}=\left\{\alpha_{0}, \cdots, \alpha_{k-1}\right\}, \quad I_{2}=\left\{\alpha_{k}, \cdots, \alpha_{k-1}\right\}, \quad I_{3}=\left\{\alpha_{\kappa}, \cdots, \alpha_{n}\right\}
$$

There exist natural numbers $l_{0}, \cdots, l_{\kappa}$ such that

$$
l_{0} \alpha^{(0)}+\cdots+l_{k-1} \alpha^{(k-1)}<l_{k} \alpha^{(k)}+\cdots+l_{k-1} \alpha^{(k-1)}
$$

and

$$
l_{0}+\cdots+l_{k-1}=l_{k}+\cdots+l_{\kappa-1} .
$$

Proof. Since $\alpha^{(i)}$ 's form a simplex in $\mathbf{N}_{0}^{n}$, there exists a unique relation for $\beta_{i} \in \mathbb{Q}$

$$
\beta_{0} \alpha^{(0)}+\cdots+\beta_{n} \alpha^{(n)}=0
$$

up to a constant multiple. Superscripts of $\alpha$ are so chosen that the following conditions for $k$ and $k(0 \leq k<\kappa \leq n)$ are satisfied: $1^{\circ}$ The coefficients $\beta_{0}, \cdots, \beta_{k-1}$ are non-zero and $\beta_{\kappa}=\cdots=\beta_{n}=0.2^{\circ} \beta_{0}, \cdots, \beta_{k-1}$ have the same sign each other and $\beta_{k}, \cdots, \beta_{\kappa-1}$ have also the same sign which is opposite to that of $\beta_{0}, \cdots, \beta_{k-1}$, and $3^{\circ}\left|\beta_{0}+\cdots+\beta_{k-1}\right|>\left|\beta_{k}+\cdots+\beta_{k-1}\right|$. (This is really an inequality because if this is an equality, $\left\{\alpha^{(0)}, \cdots, \alpha^{(\kappa-1)}\right\}$ must lie in some hyperplane.)

Let us tentatively assume $\beta_{0}, \cdots, \beta_{k-1}<0$. Then equation (3) becomes

$$
\left(-\beta_{0}\right) \alpha^{(0)}+\cdots+\left(-\beta_{k-1}\right) \alpha^{(k-1)}=\beta_{k} \alpha^{(k)}+\cdots+\beta_{\kappa-1} \alpha^{(\kappa-1)} .
$$

We choose rational numbers $\gamma_{0}, \cdots, \gamma_{k-1}$ so that $0<\gamma_{i} \leq\left(-\beta_{i}\right)$ and

$$
\tau_{0}+\cdots+\tau_{k-1}=\beta_{k}+\cdots+\beta_{k-1}
$$

We can cancell denominators in this equation by multiplying some natural number $N$. We set $l_{i}=N \gamma_{i}$ for $(0 \leq i \leq k-1) l_{i}=N \beta_{i}$ for $(k \leq i \leq \kappa-1)$. Then, it is easy to see that condition (2) of 2.13 clearly holds and condition (1) thus follows from the very choice of $\gamma_{i}$ 's. Q.E.D.

Definition 2. 14. Let $f$ be of simplex type. Then we divide $\operatorname{inl}(f)$ into three subsets according to Proposition 3.13, inl $(f)$ $=I_{1} \cup I_{2} \cup I_{3}$, and superscripts of $\alpha$ are also chosen as indicated there.

We say that "a vector field $X$ is associated with a hyperplane $h(x)$ $=1 "$, if $X=\sum a^{i} x_{i} D_{i}$ and $h(x)=\sum a^{i} x_{i}$. If $\operatorname{inl}(f)$ is a simplex, there 
exists for each vertex $\alpha^{(i)}$ the unique face of that simplex which does not contain $\alpha^{(i)}$. Let $h_{i}(x)=1$ be its defining equation, and $X_{i}$ be a vector field associated with it. When $h_{j}(x)$ is of the form $h_{j}(x)$ $=\sum a_{j}^{i} x_{i}$, we put $c_{j}=\sum_{j} a_{j}^{i} \alpha_{i}^{(j)}-1$. Then it holds that

$$
\begin{aligned}
& X_{j} x^{\alpha^{(j)}}=\left(1+c_{j}\right) x^{\alpha(j)} \\
& X_{j} x^{\alpha(\imath)}=x^{\alpha(i)}, \quad i \neq j
\end{aligned}
$$

Hence we have

$$
X_{j}\left(\sum x^{\alpha(i)}\right)=\left(\sum x^{a(i)}\right)+c_{j} x^{\alpha(j)} .
$$

The following theorem says that $\mathscr{g}(s)$ contains a noteworthy element in it.

Theorem 2. 15. There exists an element $P(s)=P(s, x, \vartheta)$ in $\mathcal{F}(s)$ which has the form

$$
P(s)=P_{k}(s) \cdots P_{\kappa-1}(s)+Q(s, x, \vartheta),
$$

with

$$
P_{j}(s)=\prod_{\nu=0}^{l_{j}-1}\left(s-X_{j}+\nu c_{j}\right)
$$

and

$$
Q(s, 0, \vartheta)=0, \operatorname{ord}^{T} Q \leq \operatorname{ord}^{T} P
$$

Thus

$$
\operatorname{ord}^{T}(P)=l_{0}+\cdots+l_{k-1}=l_{k}+\cdots+l_{k-1}=l
$$

and

$$
L(f) \leq l
$$

We used the notation

$$
\vartheta=\left(\vartheta_{1}, \cdots, \vartheta_{n}\right)=\left(x_{1} D_{1}, \cdots, x_{n} D_{n}\right) .
$$

To prove 2.15 , we here prepare two lemmata.

Lemma 2. 16. Let $f$ be of simplex type. Then, there exist $L_{j}(s, x, \vartheta)$ of total order one which has the form 


$$
L_{j}(s, x, \vartheta)=\left(s-X_{j}\right)+l_{j}(s, x, \vartheta)
$$

with

$$
l_{j}(s, 0, \vartheta)=0
$$

and satisfies

$$
L_{j}(s, x, \vartheta) f^{s}=\left(-a_{j}^{0} c_{j}\right) x^{a^{(j)}} s f^{s-1}
$$

Here, we write $a_{j}^{n}=a_{j}(0) \quad(\neq 0)$, when $f=\sum_{j=0}^{n} a_{j}(x) x^{a^{(j)}}$.

Proof. It follows from the definition of $X_{j}$ that

$$
\left(f-X_{j} f\right)=-a_{j}^{0} c_{j} x^{a^{(j)}}+\sum b_{k}^{j} x^{a^{(k)}}, b_{k}^{j} \in \mathfrak{u t} .
$$

We regard these as equations in $x^{a(k)}$ and get the solution

$$
-a_{j}^{0} c_{j} x^{a(j)}=\left(f-X_{j} f\right)+\sum c_{j}^{k}\left(f-X_{k} f\right), c_{j}^{k} \in \mathfrak{n t} .
$$

Then, $L_{j}(s, x, \vartheta)$ is given by

$$
L_{j}(s, x, \vartheta)=\left(s-Y_{j}\right)+\sum c_{j}^{k}\left(s-X_{k}\right)
$$

Q.E.D.

Lemma 2.17. Let $P$ and $Q$ satisfy the equations

$$
\begin{aligned}
& P(s, x, \vartheta) f^{s}=p(s) x^{\alpha} \varphi(x) f^{s-l}, \\
& Q(s, x, \vartheta) f^{s}=q(s) x^{\beta} \psi(x) f^{s-m},
\end{aligned}
$$

for some analytic functions $\varphi$ and $\psi$, and polynomials in $s, p$ and $q$. Then,

$$
\begin{aligned}
& Q(s-l, x, \vartheta-\alpha) P(s, x, \vartheta) f^{s} \\
& \quad=p(s) q(s-l) x^{\alpha+\beta} \varphi \psi f^{s-l-m}+p(s) x^{\alpha} \sum \frac{D^{\nu} \varphi}{\nu !}\left(Q^{(\nu)}(s-l, x, \vartheta) f^{s-l}\right) .
\end{aligned}
$$

Here we have used the notation

$$
R^{(\nu)}=\left[\left(\frac{\partial}{\partial \xi}\right)^{\nu}\left(\sum a_{\alpha} \hat{\xi}^{\alpha}\right)\right]_{\xi \mapsto D} \text {, when } R(s, x, D)=\sum a_{\alpha} D^{\alpha} .
$$

The prool is straightforward. 
Proof of Theorem 2.15. Let us set

$$
\begin{gathered}
Q_{j}(s)=L_{j}\left(s-\left(l_{j}-1\right), x, \vartheta-\left(l_{j}-1\right) \alpha^{(j)}\right) \cdots \\
\cdots L_{j}\left(s-1, x, \vartheta-\alpha^{(j)}\right) L_{j}(s, x, \vartheta) .
\end{gathered}
$$

It follows from Lemmata 2.16 and 2.17 that

$$
Q_{j}(s)=P_{j}(s)+q_{j}(s, x, \vartheta)
$$

with $q_{j}(s, 0, \vartheta)=0$, and

$$
Q_{j}(s) f^{s}=\left(-a_{j}^{0} c_{j}\right)^{l_{j}} x^{l_{j} \alpha^{(j)}}(s)_{l_{j}} f^{s-l_{\jmath}} .
$$

Since $X_{k} x^{\alpha^{(j)}}=x^{\alpha^{(j)}}$ for $k \neq j$, we have

$$
\begin{aligned}
& Q_{0}(s) Q_{1}(s) \cdots Q_{k-1}(s) f^{s}=\prod_{0}^{k-1}\left(-\alpha_{j} c_{j}\right)^{l_{j}} x^{\Sigma l_{j} \alpha^{(j)}}(s)_{l} f^{s-l}, \\
& Q_{k}(s) \cdots Q_{k-1}(s) f^{s}=\prod_{k}^{k-1}\left(-\alpha_{j} c_{j}\right)^{l_{j}} x^{\Sigma l_{j} a(j)}(s)_{l} f^{s-l} .
\end{aligned}
$$

On the other hand, $x^{\substack{k-1 \\ \Sigma} l_{j} \alpha^{(j)}}$ divides $x^{\underset{\Sigma}{\sum_{k}^{-1}} l_{j} \alpha^{(j)}}$ by (2) of 2.11. Hence, if we put

$$
P(s)=Q_{k}(s) \cdots Q_{k-1}(s)-c x^{\frac{k-1}{\sum} l_{j} \alpha^{(j)}-{ }_{0}^{k-1} \Sigma l_{j \alpha}(j)} Q_{0}(s) \cdots Q_{k-1}(s),
$$

where

$$
c=\prod_{k}^{k-1}\left(-\alpha_{j} c_{j}\right)^{l_{j}} / \prod_{0}^{k-1}\left(-\alpha_{j} c_{j}\right)^{l_{j}}
$$

$P(s)$ turns out to belong to $g(s)$ and has the required properties.

Q.E.D.

An important example of function of simplex type is the following.

Example 2.18.

$$
f(x)=\sum_{i=1}^{n} x_{i}^{n_{i}}+x_{1}^{m_{1}} \cdots x_{n}^{m_{n}}
$$

for $1 \leq m_{i}<n_{i}$. Let us put $c=\sum \frac{m_{i}}{n_{i}}-1$. When $c=0, f$ is weighted homogeneous. When $c \neq 0$, there are two cases:

i) For $c<0, I_{1}=\left\{\alpha^{(0)}\right\}$, where $\alpha^{(0)}=\left(m_{1}, \cdots, m_{n}\right), I_{2}=\left\{\alpha^{(1)}, \cdots, \alpha^{(n)}\right\}$, where $\alpha^{(i)}=\left(0, \cdots, n_{i}, \cdots, 0\right), I_{3}=\varnothing$. In this case $X_{0}=\sum \frac{1}{n_{i}} \vartheta_{i}, X_{i}=X_{0}$ 
$-\frac{c}{m_{i}} \vartheta_{i}$ and $c_{0}=c, c_{i}=-\left(n_{i} / m_{i}\right) c, 1 \leq i \leq n$.

ii) For $c>0$, the convention of superscripts in 2.13 tells $I_{1}=\left\{\alpha^{(0)}, \cdots\right.$, $\left.\alpha^{(n-1)}\right\}$ and $I_{2}=\left\{\alpha^{(n)}\right\}$. Tentatively, we change the superscripts in such a way that $I_{1}=\left\{\alpha^{(1)}, \cdots, \alpha^{(n)}\right\}, I_{2}=\left\{\alpha^{(0)}\right\}$. Then all operators become of the same form as in i).

In both cases, we can take $l=\min _{i}\left(N_{i}\right)$ where $N_{i}=\frac{1}{n_{i}} \prod_{j=1}^{n} n_{j}$. The proof is omitted.

We finally note that in Example 2.18, the $P(s)$ in Theorem 2.14 is given by

i) $\quad P(s)=\prod_{\nu=0}^{l-1}\left(s-X_{0}+\nu c\right)+Q(s), \quad$ if $\quad c>0$

ii) $P(s)=\prod_{j=1}^{n} P_{j}(s)+Q(s)$,

$$
P_{j}(s)=\prod_{\nu=0}^{l_{j}-1}\left(s-X_{j}+\nu c_{j}\right), \quad \text { if } \quad c<0
$$

In particular we can take $l_{j}=1, l=n$ if $n_{i} \geq n m_{i}(\forall i)$.

\section{$\S 6$. Generators of $g(s)$}

In this section, we give a way of explicitly determining $\mathscr{f}(s)$. We always assume that $f$ has an isolated singularity.

The ideal $g_{0}=\mathscr{g}(s) \cap \mathscr{D}$ previously defined is determined by

Theorem 2. 19 .

$$
\mathscr{g}_{0}=\sum_{i<j} \mathscr{D} X_{i j}, X_{i j}=f_{i} D_{j}-f_{j} D_{i}
$$

To prove this, we begin by stating an algebraic lemma (cf. [12]).

Lemma 2. 20. i) The following conditions 1 through 3 on $g_{i}$ $(i=1, \cdots, n)$ are equivalent.

1. $g_{i}$ is not a zerodivisor of $\mathcal{O} / \sum_{j=0}^{i-1} g_{j} \mathcal{O}, i=1, \cdots, n$, where we understand $g_{0}=0$. 
2. $\bigoplus_{\nu \geq 0} \mathfrak{a}^{\nu} / \mathfrak{a}^{\nu+1}$ is isomorphic to $\mathcal{O} / \mathfrak{a}\left[\xi_{1}, \cdots, \xi_{n}\right]$ under the homomorphism $\hat{\xi}_{i} \mapsto g_{i}$, where $\mathfrak{a}=\sum_{i=1}^{n} g_{i} \mathcal{O}$.

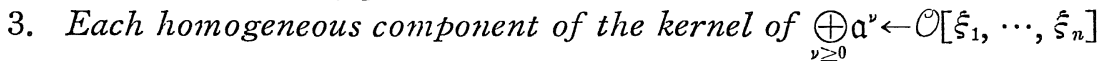
is generated by $g_{i} \xi_{j}-g_{j} \xi_{i}$ as an $\mathcal{O}[\xi]$-module. ii) If m-primary ideal $a$ of $\mathcal{O}$ is generated by n-elements $g_{1}, \cdots, g_{n},\left(g_{1}, \cdots, g_{n}\right)$ satisfies the conditions in i).

This lemma is the one known in the theory of local rings. When $\left(g_{1}, \cdots, g_{n}\right)$ satisfies one of the conditions in Lemma 3.2 i), it is called an $\mathcal{O}_{\text {-sequence. }}$

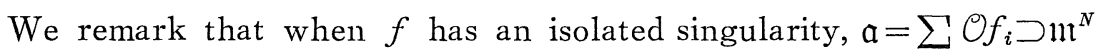
holds for large $N$ (actually, we can take $N=\mu=\operatorname{dim} \Theta / \mathfrak{a}$ ). Thus $\left(f_{1}, \cdots, f_{n}\right)$ makes an $\mathcal{O}$-sequence.

Next, choose an element $P(x, D)$ from $\mathscr{g}_{0}$ with ord $P=m$. Then the equation

$$
0=P(x, D) f^{s}=\sigma_{m}(P)(x, d f)(s)_{m} f^{s-m}+(\text { lower order in } s)
$$

readily yields $\sigma_{m}(P)(x, d f)=0$. Since $\left(f_{1}, \cdots, f_{n}\right)$ is an $\mathcal{O}_{\text {-sequence, }}$

$$
\sigma_{m}(P)(x, \tilde{\xi})=\sum q_{i j}(x, \xi)\left(f_{i} \xi_{j}-f_{j} \xi_{i}\right)
$$

by 3 of Lemma 2.20. Thus, choosing $Q_{i j} \in \mathscr{D}$ such that $\sigma^{T}\left(Q_{i j}\right)=q_{i j}$, we have

$$
\operatorname{ord}\left(P-\sum Q_{i j}(x, D) X_{i j}\right)<m \text {. }
$$

Hence by induction on $\operatorname{ord}^{T}(P)$, we complete the proof of Theorem 2.19.

Q.E.D.

When $f$ is quasi-homogeneous, the relation $s-X_{0} \in \mathcal{g}(s)$ holds with a vector field $X_{0}$ such that $X_{0} f=f$. Then for any $P(s)=\sum s^{j} P_{j}(x, D)$ $\in \mathscr{D}[s], P(s)$ and $P_{j}(x, D) X_{0}^{j}$ are congruent modulo $\mathscr{g}(s)$. Hence

$$
\begin{aligned}
& \Re \simeq \mathscr{D} / \mathscr{g}_{0}, \\
& \mathscr{M} \simeq \mathscr{D} /\left(\mathscr{g}_{0}+\mathscr{D} f\right) .
\end{aligned}
$$

Next, since $\mathscr{g}_{0} \subset \mathscr{D} \mathfrak{a}$ and $f \in \mathfrak{a}$, we have

$$
\widetilde{\pi} \simeq \mathscr{D} / \mathscr{D} \mathfrak{a}
$$


by use of Proposition 2.2 i).

When $f$ is not quasi-homogeneous, there are $a_{\nu}(x, \xi)=\sum a_{\nu, i}(x) \xi_{i}$ such that

$$
a_{\nu}(x) f+a_{\nu}(x, d f)=0,
$$

where $\left(a_{1}(x), \cdots, a_{r}(x)\right)$ are the generators of $\mathfrak{a}: f$. Set

$$
A_{\nu}(s, x, D)=a_{\nu}(x) s+a_{\nu}^{\prime}(x, D),
$$

where $a_{\nu}^{\prime}(x, D)=\sum a_{\nu, i}(x) D_{i}$. Then we have the following:

\section{Theorem 2.21.}

$$
\mathscr{I}(s) \cap(\mathscr{D} s+\mathscr{D})=\sum_{\nu=1}^{r} \mathscr{D} A_{\nu}(s, x, D)+\mathscr{g}_{0} .
$$

Proof. The proof will be carried out by induction on $l=\operatorname{ord} P(s)$ where $P(s) \in g(s)$. The case $l=0$ is trivial. There are three cases when $P(s)=Q s+R$.

1. If $\sigma_{l}^{T}(P)=\sigma_{l-1}(Q) s$, then $P f^{s}=0$ yields $\sigma_{l-1}(Q)(x, d f) f=0$. Hence

$$
\sigma_{l-1}(Q)(x, \xi) \in \sum \mathcal{O}[\xi]\left(f_{i} \dot{\xi}_{j}-f_{j} \hat{\xi}_{i}\right),
$$

and we can lower $\operatorname{ord}^{T}(P)$ by subtracting from $P$ a suitable element in $\mathcal{I}_{0} \cdot s$.

2. If $\sigma_{l}^{T}(P)=\sigma_{l}(R)$, then, $\sigma_{l}(R)(x, d f)=0$. The proof in this case reduces to that in 1 , if we replace $\mathscr{G}_{0} \cdot s$ in 1 by $\mathcal{G}_{0}$.

3. If $\sigma_{l}^{T}(P)=\sigma_{l-1}(Q) s+\sigma_{l}(R)$, then

$$
\sigma_{l-1}(Q)(x, d f) f+\sigma_{l}(R)(x, d f)=0 .
$$

Denote $\sigma_{l-1}(Q)(x, \xi)=\sum_{|\alpha|=l-1} a_{(\alpha)} \hat{\xi}^{\alpha}$. Then the statement 2 in 2.20 and (7) yield $a_{(\alpha)} f \in \mathfrak{a}$. Thus we can find $b_{1}^{(a)}, \cdots, b_{r}^{(a)}$ such that

$$
a_{(a)}=\sum_{\nu} b_{\nu}^{(a)}(x) a_{\nu}(x) .
$$

Hence,

$$
P^{\prime}(s)=P(s)-\sum_{\nu}\left(\sum_{\alpha} b_{\nu}^{(a)}(x) D^{\alpha}\right) A_{\nu}(s, x, D)
$$

is either of total order less than $l$ or reduces to the $P(s)$ in case 2 .

Obviously, $\quad y_{0} \cdot s \subset \Sigma^{\prime} D A_{\nu}+y_{0}$ since $a: f \supset \mathfrak{a}$. Thus we have com- 
pleted the proof of Theorem.

Q.E.D.

We now proceed to the determination of the structure of $g(s)$ when $L(s)=2$. Since $s^{2}+A s+B \in \mathscr{g}(s)$ in this case, every element in $\mathscr{D}[s]$ is congruent to an element in $\mathscr{D} s+\mathscr{D}$ modulo $\mathscr{g}(s)$. Therefore we obtain the following:

Corollary 2. 22. When $L(f)=2, g(s)$ is generated by $g_{0}$ together with $A_{\nu}(s, x, D)$ and $s^{2}+s A+B$.

Modules $\mathscr{M}, \mathscr{M}$ and $\widetilde{\mathscr{M}}$ are generated by two elements $\bar{l}$ and $\bar{s}$, where the bar indicates the residue class of the element without bar. Their structure is characterized by the following theorem.

Theorem 2.23. Modules $\mathfrak{N}, \mathcal{M}$ and $\widetilde{M}$ have following presentations.

i) When $L(f)=1$,

$$
\begin{aligned}
& 0 \leftarrow \mathscr{M} \leftarrow \mathscr{D} \stackrel{\left(X_{i j}\right)}{\longleftarrow} \mathscr{D}^{\left(\begin{array}{l}
n \\
2
\end{array}\right)}, \\
& 0 \leftarrow \mathscr{M} \leftarrow \mathscr{D} \stackrel{\left(\begin{array}{c}
X_{i j} \\
f
\end{array}\right)}{\longleftarrow} \mathscr{D}^{\left(\begin{array}{l}
n \\
2
\end{array}\right)+1}, \\
& 0 \leftarrow \widetilde{M} \leftarrow \mathscr{D} \stackrel{\left(f_{i}\right)}{\longrightarrow} \mathscr{D}^{n} .
\end{aligned}
$$

ii) When $L(f)=2$,

$$
0 \leftarrow \mathscr{M} \stackrel{\left(\begin{array}{l}
1 \\
s
\end{array}\right)}{\longleftarrow} \mathscr{D}^{2} \stackrel{\left(\begin{array}{ll}
X_{i j} & 0 \\
a_{\nu}^{\prime} & a_{\nu}
\end{array}\right)}{\longleftarrow} \mathscr{D}^{\left(\begin{array}{l}
n \\
2
\end{array}\right)+r},
$$

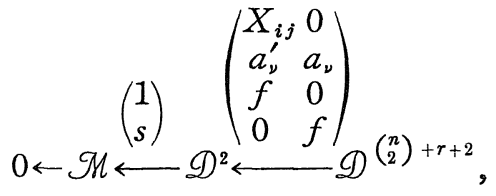

$$
0 \leftarrow \widetilde{M} \stackrel{\left(\begin{array}{l}
1 \\
s
\end{array}\right)}{\longleftarrow} \mathscr{D}^{2} \stackrel{\left(\begin{array}{ll}
f_{i} & 0 \\
f & 0 \\
a_{\nu}^{\prime} & a_{\nu}
\end{array}\right)}{D^{n+r+1}} .
$$


Remark: We can easily show that the sequence

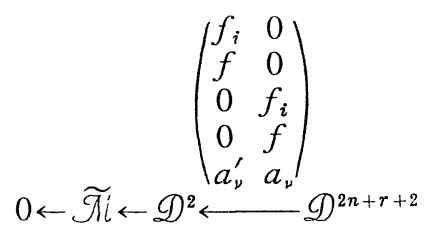

is exact. It is the absence of $f_{i}$ and $f$ in the right column of the map in (13) that plays an important role in the following sections.

Proof of Theorem 2.23. We first note that sequences (8), (9) and (10) are direct consequences of (4), (5) and (7), Sequence (11) is derived from Theorem 2.20. Let us try to prove (13). Sequence (12) can be proved in a similar way.

First, suppose $P \overline{1}+Q \bar{s}=0$. Then there are $R_{i}(s)$ and $S(s)$ such that

$$
(P+Q s) f^{s}=\sum R_{i}(s) f_{i} f^{s}+S(s) f^{s+1},
$$

where the first term of the right-hand side can further be written

$$
\begin{aligned}
R_{i}(s) f_{i} f^{s} & =\left(R_{i}^{\prime}(s)(s+1)+R_{i}(-1)\right) f_{i} f^{s} \\
& =R_{i}^{\prime}(s) D_{i} f^{s+1}+R_{i}(-1) f_{i} f^{s} .
\end{aligned}
$$

Second, set $S^{\prime}(s)=S(s)+\sum R_{i}^{\prime}(s) D_{i}$. Then, by making use of

$$
\left((s+1)^{2}+(s+1) A+B\right) f^{s+1}=0,
$$

we find $S_{1}$ and $S_{2} \in \mathscr{D}$ which satisfy

$$
S^{\prime}(s) f^{s+1}=\left(S_{2}+S_{1} s\right) f^{s+1} .
$$

Hence we have

$$
(P+Q s) f^{s}=\sum R_{i}(-1) f_{i} f^{s}+\left(S_{2}+S_{1} s\right) f^{s+1} .
$$

Next set $\sigma^{T}\left(s^{2}+s A+B\right)=s^{2}+\left(\sum a_{i} \xi_{i}\right) s+\left(\sum a_{i j} \xi_{i} \xi_{j}\right)$. Then, $\sum a_{i j} f_{i j}$ $=\sum b_{i} f_{i}+b f$ for some $b_{i}$ and $b$ by Corollary 2.5. Since $f^{2}+\left(\sum a_{i} f_{i}\right) f$ $+\sum a_{i j} f_{i} f_{j}=0$, we have

$$
f s+\sum a_{i} f D_{i}+\sum a_{i j} f_{i} D_{j} \in \mathcal{G}(s) .
$$

It follows that

$$
s f^{s+1}=\left[\sum_{i}\left(a_{i}+b_{i}-\sum_{i} a_{i j} D_{j}\right) f_{i}-\left(\sum a_{i} D_{i}-b\right) f\right] f^{s} .
$$


Consequently, there are $R_{i}$ and $S \in \mathscr{D}$ such that

$$
(P+Q s) f^{s}=\left(\sum R_{i} f_{i}\right) f^{s}+S f^{s+1} .
$$

This formula and Theorem 2.20 implies (13) since $\mathscr{g}_{0} \subset \mathscr{D} \mathfrak{a}$. Q.E.D.

When $\operatorname{dim} X=2$, the statement in Theorem 2.21 can be made simpler with the aid of Corollary 1.11. First note that $f\left(x_{1}, x_{2}\right)$ is locally reduced since we assume $\mathfrak{a} \supset \mathfrak{m}^{N}$.

When $L(f)=1, g(s)$ is generated by $s-X_{0}$ and $X_{12}$, (or $\mathcal{G}$ is generated by $X_{0}$ and $X_{12}$ ).

When $L(f) \geq 2, \mathfrak{a}: f$ is generated by two elements anounced in Theorem 1.10 since so is $\mathcal{G}$. Let $\mathfrak{a}: f=\mathcal{O} a_{1}+\mathcal{O} a_{2}$ and $A_{\nu}(s, x, D)$ $=a_{\nu}(x) s+\left(a_{\nu 1}(x) D_{1}+a_{\nu 2}(x) D_{2}\right)$. Then $X_{12}$ must be represented by $A_{1}$ and $A_{2}$. Since $\left(a_{1}, a_{2}\right)$ is also of an $\mathcal{O}$-sequence, the relations between $a_{1}$ and $a_{2}$ are generated by the following trivial relation:

$$
\left(-a_{2}\right) a_{1}+a_{1} a_{2}=0 .
$$

Since $g_{0}=\mathscr{D} X_{12}$, we obtain

$$
a_{1} A_{2}-a_{2} A_{1}=\varphi X_{12}
$$

where $\varphi(0) \neq 0$. Now we restate Theorem 2.21 in the form:

Theorem 2. 24. Suppose $\operatorname{dim} X=2$. Then,

$$
g(s) \cap(\mathscr{D} s+\mathscr{D})=\sum_{\nu=1}^{2} \mathscr{D} A_{\nu}(s, x, D),
$$

where $A_{\nu}(s, x, D)=a_{\nu}(x) s+\left(a_{\nu 1}(x) D_{1}+a_{\nu 2}(x) D_{2}\right)$ with $\mathfrak{a}: f=\mathcal{O} a_{1}+\mathcal{O} a_{2}$. (When $f$ is quasi-homogeneous, we understand $a_{1}=1$ and $a_{2}=0$.)

Conversely, if there exist in $\mathscr{g}(s) \cap(\mathscr{D} s+\mathscr{D}), A_{1}$ and $A_{2}$ with above form such that $\operatorname{det}\left[\begin{array}{ll}a_{11} & a_{12} \\ a_{21} & a_{22}\end{array}\right]=\varphi f, \varphi(0) \neq 0$, then they generate $\mathscr{g}(s) \cap(\mathscr{D} s+\mathscr{D})$.

\section{§ 7. "Fundamental Conjecture" - Counter Examples-}

Let $p(s, x, \xi)$ belong to $\mathcal{O}_{X}[s, \xi]$ and be homogeneous in $(s, \xi)$ in such a way that 


$$
p(f, x, d f)=0 .
$$

It follows from Proposition 2.3 that there is an integer $l$ such that $p^{l} \in \sigma^{T}(g(s))$.

In connexion with this fact, M. Sato and M. Kashiwara once conjectured in [12] that for such $p$, at least one of the following three statements should hold.

RS $1 p \in \sigma^{T}(\mathcal{F}(s))$.

RS 2 There exists $m_{0} \in \boldsymbol{N}$ for which the following holds. $p \in \sigma^{T}(g(s))$, if $\operatorname{deg} p \geq m_{0}$.

RS 3 There exists $q(x, \xi)$ which is homogeneous in $\xi$ such that $q(0, \xi) \neq 0$ and that $q p \in \sigma^{T}(g(s))$.

However, they fail to hold in general, as is shown later.

It should be remarked that RS 1 is true for $f$ being of isolated singularity with $L(f) \leq 2$. In fact, if $p(f, x, d f)=0$, after dividing $p(s, x, \xi)$ by $s-\sum a_{i} x_{i} \xi_{i}$ or $s^{2}+\sigma_{1}(A) s+\sigma_{2}(B)$, one can use the argument of the proof of 2.18 or 2.20 to find the required operator $P(s)$.

Proposition 2. 25. 1. Let $f$ satisfy RS1. Then, there exists an operator $P(s)$ such that

$$
P(s) f^{s+1}=b(s) f^{s *)} \text { reith } \operatorname{ord}^{T} P=\operatorname{deg} b .
$$

2. Let $\int$ satisfy RS2. Then, there are $P_{\nu}(s)$ and $\nu$ such that

$$
P_{\nu}(s+\nu) f^{s+\nu}=b_{\nu}(s) f^{s} \text { roith } \operatorname{ord}^{T} P_{\nu}=\operatorname{deg} b_{\nu}
$$

Corollary 2.26. Assume that $f$ has an isolated singularity at 0 and that $L(f) \leq 2$. Then one can find a "b-operator" $P(s)$ such that $\operatorname{ord}^{T}(P)=\operatorname{deg} b$.

Proof of Proposition 2.25. Assume $\operatorname{ord}^{T} P>d=\operatorname{deg} b$. Then,

$$
\sigma^{T}(P(s) f-b(s))=\sigma^{T}(P(s)) f .
$$

Thus $\sigma^{T}(P(s))(f, x, d f)=0$. By RS 1 , there exists an operator $P^{\prime}(s)$

*) Such an operator is called a " $b$-opepator" in the sequel. 
in $g(s)$ such that $\sigma^{T}\left(P^{\prime}(s)\right)=\sigma^{T}(P(s))$. Set $P^{\prime \prime}(s)=P(s)-P^{\prime}(s+1)$. Then, $\operatorname{ord}^{T} P^{\prime \prime}<\operatorname{ord}^{T} P$ and $P^{\prime \prime}(s) f^{s+1}=b(s) f^{s}$. Proceeding in this way, we finally reach the stage that $\operatorname{ord}^{T}\left(P^{\prime \prime \prime}(s)\right)=\operatorname{deg} b$.

The Proof of 2 is much the same.

Q.E.D.

Proof of Corollary 2.26. This can be directly proved by Proposition 2.25. 1 and the argument preceding it.

We can also prove this Corollary by the aid of concrete process of determining operator $P(s)$ and by the simple fact that "If an ideal $\mathcal{G}$ in $\mathscr{D}$ is generated by elements $\left\{a_{1}, \cdots, a_{k}\right\}$ in $\mathcal{O},\left(a_{1}, \cdots, a_{k}\right)$ forms an involutory basis of $g$ ". See p. 161 and p. 163.

The following gives us a counter example against RS 1 and RS 2 .

Example 2. 27. $f(x)=\frac{1}{n}\left(x^{n}+y^{n}+z^{n}\right)-\frac{1}{m}(x y z)^{m}$

$$
n \geq 5 m-2, m \geq 2 \text {. }
$$

This is of simplex type. We set $c=\frac{3 m}{n}-1 \quad(<0)$,

$$
X_{0}=\frac{1}{n}\left(x D_{x}+y D_{y}+z D_{z}\right), X_{1}=X_{0}-\frac{c}{m} D_{x} \text { etc., and } \varphi=1-(x y z)^{n-3 m} \text {. }
$$

Let $(i, j, k)$ be a permutation of $(1,2,3)$ and define the vector field $X_{123}$, for example, by

$$
X_{123}=\frac{-1}{\varphi}\left(y^{m-1} z^{2 m-1} D_{x}+x^{n-m-1} z^{m-1} D_{y}+x^{n-2 m-1} y^{n-m-1} D_{z}\right) .
$$

Other operators are defined according to the permutation of variables. $X_{i j k}$ satisfies

$$
X_{i j k} f=x^{i m-1} y^{j m-1} z^{k m-1} .
$$

We can verify the following:

$1 \quad a \ni x^{n-1}-x^{m-1}(y z)^{m}=f_{x}, x^{m-1} y^{2 m-1} z^{3 m-1}, x^{2 m-1} y^{n-m-1}$, $x^{n+2 m-1} y^{m-1}, x^{2 n+m-1}$, etc.

$2 \mathfrak{a}: f=\left(x^{m-1} y^{2 m-1}, x^{n-m}-(y z)^{m}\right.$, etc. $)$

$3 \operatorname{dim} \mathcal{O} / \mathfrak{a}=3 n^{2}(m-1)+3 n-1$, 


$$
\begin{array}{ll} 
& \operatorname{dim} \mathcal{O}(\mathfrak{a}: f)=3 n(m-1)^{2}-(m-1)\left(m^{2}-8 m+1\right)+1, \\
4 & a+\mathcal{O} f=\left(f_{x}, f_{y}, f_{z},(x y z)^{m}\right), \\
5 & \left\{\left(a^{2}+a f\right): f^{2}\right\} /(\mathfrak{a}: f)=\left(x^{2 m-2},(x y)^{m-1}, \cdots\right) .
\end{array}
$$

Statements 1 through 4 hold even when $n \geq 3 m+1$.

The structure of $g(s)$ is as follows.

$$
\begin{aligned}
& X_{i j}, x^{m-1} y^{2 m-1}\left(s-X_{3}\right)-\frac{c}{m}\left(x^{m} y^{2 m-1} D_{x}+z X_{231}\right), \\
& \left(x^{n-m}-(y z)^{m}\right)\left(s-X_{1}\right)-\frac{c}{m} x^{n-m}\left(x D_{x}\right) \text { etc. }
\end{aligned}
$$

are generators of $\mathscr{g}(s) \cap(\mathscr{D} s+\mathscr{D}) . \quad L(f) \leq 3$ is guaranteed by the existence in $\mathscr{g}(s)$ of the operator

$$
\left(s-X_{1}\right)\left(s-X_{2}\right)\left(s-X_{3}\right)-(x y z)^{n-3 m}\left(s-X_{0}+2 c\right)\left(s-X_{0}+c\right)\left(s-X_{0}\right) .
$$

The operator in $y(s)$ with the leading term $(x y)^{m-1} s^{2}$ is

$$
(x y)^{m-1}\left(s-X_{3}\right)\left(s-Y_{3}+c\right)+\left(\frac{c}{m}\right)^{2} z^{n-m} D_{x} D_{y}+\left(\frac{c}{m}\right)^{2} T
$$

where

$$
\begin{gathered}
\mathrm{I}_{3}=X_{0}-\frac{c}{m}\left(x D_{x}+y D_{y}\right) \\
T=(x y)^{n-4 m+1} \cdot x^{n-5 m+2} X_{123} \cdot X_{321}+\frac{1}{\varphi}\left\{(x y)^{n-4 m} z^{n-4 m+1}\right. \\
\times\left((3 m-1)(y z)^{m}+(3 m-2) x^{n-m}\right) X_{321} \\
\left.+(m-1) x^{2 n-3 m-1} y^{n-3 m} z^{n-4 m} D_{y}\right\}
\end{gathered}
$$

The situation for $x^{2 m-2} \in\left(\mathfrak{a}^{2}+\mathfrak{a} f\right): f^{2}$ is delicate. We have

$$
\left(s-X_{2}\right)\left(s-X_{3}\right) f^{s}=\left(\frac{c}{m}\right)^{2}(y z)^{n} s(s-1) f^{s-2},
$$

and

$$
\begin{aligned}
& Q f^{s}=x^{m-2}(y z)^{5 m-2} s(s-1) f^{s-2}-\frac{m-1}{\varphi} x^{m-2}(y z)^{4 m-2} s f^{s-1}, \\
& \begin{aligned}
& Q=X_{123} X_{132}+\frac{1}{\varphi} x^{n-3 m-1} y^{2 m-1} z^{m-1} \\
& \quad \quad\left\{(3 m-1) X_{312}+(2 m \iota-1) y^{n-3 m} X_{231}\right\} .
\end{aligned}
\end{aligned}
$$


Then setting

$$
P(s)=x^{2 m-2}\left(s-X_{2}\right)\left(s-X_{3}\right)-\left(\frac{c}{m}\right)^{2}(y z)^{n-5 m+2} Q,
$$

we obtain

$$
P(s) f^{s}=\left(\frac{c}{m}\right)^{2} \frac{m-1}{\varphi} x^{m-2}(y z)^{n-m} s f^{s-1}
$$

On the other hand

$$
x^{m-2}(y z)^{n-m} \notin\left(x^{n-1}-x^{m-1}(y z)^{m}, \cdots,(x y z)^{m}\right)=\mathfrak{a}+\mathcal{O} f .
$$

For, if $x^{m-2}(y z)^{n-m} \in \mathfrak{a}+\mathcal{O} f$, we consider both sides $\bmod x^{m-1}$ and have $(y z)^{n-m} \in\left(y^{n-1}, z^{n-1}\right)_{\Theta_{y}, z}$, which is impossible since $m \geq 2$. Therefore, (14) gives a counter example against RS 1 by Corollary 2.5, 1). We can find, however, following three operators.

$$
\begin{gathered}
x P(s)-\left(\frac{c}{m}\right)^{2} \frac{m-1}{\varphi} y^{n-3 m+1} z^{n-4 m+1} X_{123}, \\
y^{m-1}\left\{x^{2 m-2}\left(s-X_{2}-\frac{(m-1) c}{m \varphi}\right)\left(s-X_{3}\right)-\left(\frac{c}{m}\right)^{2}(y z)^{n-5 m+2} Q\right\} \\
-\left(\frac{c}{m}\right)^{2} \frac{m-1}{\varphi} x^{m-2} z^{n-m} D_{y},
\end{gathered}
$$

and

$$
\begin{gathered}
z^{m-1}\left\{x^{2 m-2}\left(s-X_{3}-\frac{(m-1) c}{m \varphi}\right)\left(s-X_{2}\right)-\left(\frac{c}{m}\right)^{2}(y z)^{n-5 m+2} Q\right\} \\
-\left(\frac{c}{m}\right)^{2} \frac{m-1}{\varphi} x^{m-2} y^{n-m} D_{z} .
\end{gathered}
$$

Their total symbols are $x \sigma^{T}(P), y^{m-1} \sigma^{T}(P)$, and $z^{m-1} \sigma^{T}(P)$. Moreover, we can choose an element in $\mathscr{g}(s)$, whose total symbol is $\xi^{m-1} \sigma^{T}(P)$. Set

$$
\begin{aligned}
S_{m-2}= & \sum_{j=1}^{m-1}(m-1)_{j} D_{x}^{m-1-j} \frac{1}{\varphi} x^{m-1-j} \\
R(s)= & S_{m-2}(x y z)^{n-4 m} \\
& \times\left\{\left(s-X_{0}+c\right)\left(s-X_{0}\right)+\left(\frac{c}{m}\right)^{2} \frac{n-3 m}{\varphi} y^{n-2 m+1} \approx^{3 m+1} X_{123}\right\}
\end{aligned}
$$


or when $n \geq 5 m-1$, set

$$
\begin{aligned}
R(s)= & \left(\frac{c}{m}\right)^{2} S_{m-2} x^{n-4 m}(y z)^{n-5 m+1}\left[\frac{n-3 m}{\varphi} y^{n-m} z^{n-2 m} X_{123}\right. \\
& +\left\{x y z X_{123} \cdot X_{321}+\frac{x^{2 m}}{\varphi}\left((3 m-1) y^{m} X_{123}+x^{n-4 m}\right.\right. \\
& \left.\left.\times\left((2 m-1)(x z)^{m}+(m-1) y^{n-m}\right) X_{321}\right\}\right]
\end{aligned}
$$

Then,

$$
\begin{array}{r}
\left\{\left(D_{x}^{m-1} x^{2 m-2}+S_{m-2} x^{m-1}\right)\left(s-X_{2}\right)\left(s-X_{3}\right)\right. \\
\left.-D_{x}^{m-1}\left(\frac{c}{m}\right)^{2}(y z)^{n-5 m+2} Q\right\}-R(s)
\end{array}
$$

is an element in $g(s)$. Thus (14) cannot be a counter example against RS 3 .

But there are no element in $g(s)$ with its total symbol $\eta^{l_{1}} \zeta^{l_{2}} \sigma^{T}(P)$. In fact, if $D_{y}^{l_{1}} D_{z}^{l_{2}} P(s)+R^{\prime}(s) \in g(s), \operatorname{ord}^{T} R^{\prime}<l_{1}+l_{2}+2$, then

$$
\begin{aligned}
D_{y}^{l_{1}} D_{z}^{l_{2}} \varphi P(s)= & \left(\frac{c}{m}\right)^{2}(m-1) f_{y}^{l_{1}} f_{z}^{l_{2}} x^{m-2}(y z)^{n-m}(s)_{l_{1}+l_{2}+1} f^{s-1-l_{1}-l_{2}} \\
& +(\text { lower order in } s)
\end{aligned}
$$

implies

$$
x^{m-2}(v z)^{n-m} f_{y}^{l_{1}} f_{z}^{l_{2}} \in(\mathfrak{a}+\mathcal{O} f)^{l_{1}+l_{2}+1},
$$

by Corollary $2.5,1)$. If this formula were true, there should be a homogeneous polynomial $F\left(t_{1}, \cdots, t_{4}\right)$ of degree $l_{1}+l_{2}+1$ with coefficients in $\mathcal{O}$, such that

$$
x^{m-2}(y \approx)^{n-m} f_{y}^{l_{1}} f_{z}^{l_{2}}=F\left(f_{x}, f_{y}, f_{z},(z y z)^{m}\right) .
$$

Considering both sides mod $x^{m-1}$, we conclude that

$$
y^{(n-1)\left(l_{1}+1\right)-m+1} z^{(n-1)\left(l_{2}+1\right)-m+1} \in\left(\mathcal{\Theta}_{y z} y^{n-1}+\mathcal{O}_{y z} z^{n-1}\right)^{l_{1}+l_{2}+1} .
$$

But this never occurs. Thus (14) also serves a counter example against RS 2. Set

$$
P^{\prime}(s)=\frac{1}{\varphi}\left(x^{2 m-2}\left(s^{\prime}-X_{2}\right)\left(s^{\prime}-X_{3}\right)-\left(\frac{c}{m}\right)^{2}(y z)^{n-5 m+2} X_{123} X_{132}\right) \varphi
$$


where

$$
s^{\prime}=s+c-2-\frac{3}{n} .
$$

Then the following operator belongs to $g(s)$.

$$
\begin{aligned}
P^{\prime}(s) & P(s)-\frac{m-1}{\varphi}\left(\frac{c}{m}\right)^{2}(y z)^{n-5 m+2}\left[X_{123} \frac{1}{\varphi}\left(\frac{c}{m}\right) x^{m-3} y^{m-1} z^{n-2 m-1}\right. \\
& \times\left\{(m-2) z^{2 m}\left(s-X_{2}\right)+(x y)^{n-3 m}\left(z^{n-m}+x^{m} y^{m}\right)\left(s-X_{0}\right)\right\} \\
& +\frac{1}{\varphi} x^{2 m-4} y^{2 m-2} z^{m-2}\left\{(2 m-3) y^{m} z^{2 m}+(n+2 m-1) x^{n-m} z^{m}\right. \\
& \left.\left.+(n+m-1) x^{n-2 m} y^{n-m}\right\}\left(s-X_{2}\right)\left(s-X_{3}\right)\right] .
\end{aligned}
$$

Thus $\left(\sigma^{T}(P)\right)^{2} \in \sigma^{T}(\mathscr{g}(s))$.

The following gives a counter example against RS 1,2 and 3 .

Example 2. 28. $f=x^{5}(x+t y)-y^{5}, t$ : a parameter. This is a $\mu$ constant deformation of $x^{6}-y^{5} \quad(\mu=20)$. Set

$$
X_{0}=\frac{1}{6} x D_{x}+\frac{1}{5} y D_{y}, \quad X_{1}=\frac{4}{25} x D_{x}+\frac{1}{5} y D_{y} .
$$

For $t \neq 0, g(s) \cap(\mathscr{D} s+\mathscr{D})$ is generated by

$$
(6 x+5 t y)\left(s-X_{0}+\frac{t}{180} y D_{x}\right)-\frac{t^{2} y^{2}}{36} D_{x},
$$

and

$$
y^{2}\left(s-X_{0}+\frac{t}{180} y D_{x}\right)-\frac{t^{2}}{36} x X_{34}
$$

where

$$
X_{34}=\frac{1}{5}\left(1-\frac{5^{3} t^{5}}{6^{4}} x\right)^{-1}\left(\frac{(6 x)^{4}-(5 t y)^{4}}{6 x+5 t y} \cdot \frac{t}{6^{4}} D_{x}-x^{3} D_{y}\right)
$$

We set

$$
\begin{aligned}
P(s)= & \left(s-X_{0}+\frac{t}{180} y D_{x}+\frac{1}{15}\right)\left(s-X_{0}+\frac{t}{180} y D_{x}\right) \\
& -\frac{5^{3} t^{4}}{(36)^{2}} x\left(s-X_{1}-\frac{1}{15}\right)\left(t\left(s-X_{1}\right)-\frac{1}{25} x D_{y}\right) .
\end{aligned}
$$


Then

$$
P(s) f^{s}=k s(x y)^{3} f^{s-1}, k=\frac{4 t^{3}}{5 \cdot(36)^{2}} .
$$

We can verify by direct calculation that

$$
(x y)^{3} \notin \mathfrak{a}+\mathcal{O f},
$$

Formula (16) shows that (15) displays a counter example against RS 1. We can also show that this is a counter example against RS 2 by the same way as in 3.24 , considering $D_{y}^{l} P(s)$ and $\bmod x^{4}$.

Lastly, we show that this is a counter example against RS 3. Our argument relies on the following general proposition.

Proposition 2.29. 1. Let $f$ have an isolated singularity at 0 with $l(f)=2$, and let $P(s)$ satisfy $\operatorname{ord}^{T}(P)=m \geq 2$, and

$$
P(s) f^{s}=s^{m-1} a(x) f^{s-m+1}+(\text { lower order in } s) .
$$

If there exists $Q(x, D) \in \mathscr{D}$ such that $\sigma(Q)(0, \hat{\varsigma}) \neq 0$, and

$$
\sigma^{T}(Q(x, D) P(s)) \in \sigma^{T}(g(s)),
$$

then $a(x) \in \mathfrak{a}+\mathcal{O f}$ when $m=2$, or $a(x) \in \mathfrak{a}$ when $m \geq 3$.

Especially when $m=2$, if (17) gives a counter example against $R S 1$, then it is also a counter example against $R S 3$.

2. More generally, if $l(f)=\lambda \geq 2$ and $\operatorname{ord}^{T} P \geq \lambda, \quad a(x) \in \mathfrak{a}+\mathcal{O} f^{\lambda-1}$, when $\operatorname{ord}^{T}(P)=\lambda$, or $a(x) \in \mathfrak{a}$ when $\operatorname{ord}^{T}(P)>\lambda$.

Remark: This proposition is useful only when $m=\operatorname{ord}^{T}(P)=l(f)$ and $l(f)+1$. Since, regardress of the existence of $Q$, we see

$$
a(x) \in\left(\mathfrak{a}+\mathcal{O} f+\sum \mathcal{O} f_{i j}\right)(\mathfrak{a}+\mathcal{O} f)^{m-2} .
$$

Thus $a(x) \in \mathfrak{a}$ when ord $P \geq l(f)+2$.

Proof. Since $f^{2} \in \mathfrak{a}^{2}+\mathfrak{a} f$, we can show by induction on $k$ that

$$
(\mathfrak{a}+\mathcal{O} f)^{k}=\mathfrak{a}^{k-1}(\mathfrak{a}+\mathcal{O} f) .
$$

Now, if $\sigma^{T}(Q P) \in \sigma^{T}(\mathscr{g}(s))$,

$$
\sigma_{l}(Q)(x, d f) a(x) \in(\mathfrak{a}+\mathcal{O} f)^{l+m-1}=\mathfrak{a}^{l+m-2}(\mathfrak{a}+\mathcal{O} f) .
$$


Therefore, since $m \geq 2$, there is a homogeneous polynomial $p_{l}(x, \xi)$ of degree $l$ with coefficients in $\mathfrak{a}^{m-2}$ such that

$$
\sigma_{l}(Q)(x, d f) a(x)+p_{l}(x, d f) f \in \mathfrak{a}^{l+m-1} .
$$

$\mathrm{By}$ the aid of Theorem 2.19, 2 and $m \geq 2$, all the coefficients of $a(x) \sigma_{l}(Q)(x, \xi)+f p_{l}(x, \xi)$ are in $a$. Thus

$$
a(x) \in \mathfrak{a}+\mathfrak{a}^{m-2} f,
$$

owing to the condition $\sigma(Q)(0, \xi) \neq 0$.

The proof of 2 is almost the same.

Q.E.D.

We apply 2.29 1 to (15). Then (16) implies that (15) is a counter example against RS 3 .

There is $Q(s) \in \mathcal{G}(s)$ which assures $L(f) \leq 3$.

$$
\begin{aligned}
Q(s)= & \left(s-X_{0}+\frac{1}{10}\right)\left(s-X_{0}+\frac{1}{15}\right)\left(s-X_{0}+\frac{t}{180} y D_{x}\right) \\
& -\frac{5^{3} t^{5}}{6^{4}} x\left(s-X_{1}-\frac{4}{15}\right)\left(s-X_{1}-\frac{1}{15}\right)\left(s-X_{1}-\frac{1}{25 t} x D_{y}\right) .
\end{aligned}
$$

Therefore, our $f$ has the property that $2=l(f)<L(f)=3$. (cf. § 8) $g(s)$ is generated by (a), (a'), (c) and the following operator:

$$
y P(s)-k X_{34} \text {. }
$$

Set

$$
\begin{aligned}
P^{\prime}(s)= & \left(s-X_{0}+\frac{t}{180} y D_{x}+\frac{1}{6}\right)\left(s-X_{0}+\frac{t}{180} y D_{x}+\frac{1}{10}\right) \\
& -\frac{5^{3} t^{4}}{6^{4}} x\left(s-X_{1}+\frac{1}{25}\right)\left(t\left(s-X_{1}+\frac{2}{25}\right)-\frac{1}{25} x D_{y}\right) .
\end{aligned}
$$

Then

$$
\begin{aligned}
& P^{\prime}(s) P(s)-\frac{k t}{12}\left(s-X_{0}+\frac{t}{180} y D_{x}+\frac{1}{6}\right) x\left(t\left(s-X_{1}\right)-\frac{1}{25} x D_{y}\right) \\
& -\frac{35 k t^{3}}{6^{3}} y\left(s-X_{1}\right)\left(s-X_{2}\right)-\frac{25 k t^{2}}{12} x\left(s-X_{1}+\frac{1}{25}\right)\left(s-X_{0}+\frac{t}{180} y D_{x}\right)
\end{aligned}
$$

is an element in $g(s)$. Thus $\left(\sigma^{T}(P)\right)^{2} \in \sigma^{T}(g(s))$.

We shall show later that several types of polynomials give us 
concrete counter examples against RS 1 3 (§18).

The next example satisfies $l(f)=3$. Corresponding operator of total order 3 fulfills conditions $1^{\circ}$ and $2^{\circ}$ in Theorem 2.1 , but violates condition $3^{\circ}$.

Example 2. 30. $f=\frac{1}{n_{1}} x^{n_{1}}+\frac{1}{n_{2}} y^{n_{2}}-t x^{n_{1}} y^{n_{2}-1}$.

$c=\frac{m_{1}}{n_{1}}-\frac{1}{n_{2}}, c^{\prime}=\frac{n_{1}-m_{1}}{n_{1}\left(n_{2}-1\right)}-\frac{1}{n_{1}}, t$ is a parameter. We assume $c>0$ and $\left(n_{1}-1\right) / 5 \leq m_{1} \leq\left(n_{1}-2\right) / 4$. Consider the following operator:

$$
\begin{aligned}
P(s)= & \left(s-X_{0}+4 c-c t x^{m_{1}} D_{y}\right)\left(s-X_{0}+2 c-c t x^{m_{1}} D_{y}\right) \\
& \times\left(s-X_{0}-c t x^{m_{1}} D_{y}\right)-m_{1} m_{2}{ }^{6} t^{7} x^{5 m_{1}-n_{1}+1} y^{n_{2}-7} \\
& \times\left\{x^{m_{1}}\left(s-X_{2}+c^{\prime}+c_{2}\right)-3 \frac{\left(n_{2}-2\right) c}{m_{2}^{2} t} y\right\} \\
& \times\left(s-X_{2}+c^{\prime}\right)\left(x^{m_{1}-1}\left(s-X_{2}\right)+\frac{c}{m_{1} m_{2} t} y D_{x}\right) .
\end{aligned}
$$

It follows that

$$
P(s) f^{s}=c^{3} t^{3}\left(n_{2}-1\right)\left(n_{2}-2\right) a(x) s f^{s-1}
$$

where

$$
a(x)=-x^{3 m_{1}} y^{n_{2}-3}+\left(n_{2}-3\right) t x^{4 n_{1}} y^{n_{2}-4} .
$$

Thus conditions $1^{\circ}$ and $2^{\circ}$ of Theorem 2.4 hold for $\sigma^{T}(P)$.

We prove that (19) serves a counter example against RS 1 and RS 2 using condition $3^{\circ}$. Consider the operator $D_{x}^{l} P(s)$. If RS 2 holds for $\sigma^{T}(P)$, the following must hold by condition $3^{\circ}$, for $l \gg 0$.

$$
\int_{x}^{l} a(x) \in(\mathfrak{a}+\mathcal{O} f)^{l+1}+\mathfrak{c}_{l+2} .
$$

Claim: $\quad \mathfrak{c}_{l_{1}, 2} \subset \mathcal{O} \cdot x^{\left(n_{1}-1\right)(l+1)}+\mathcal{O} y^{n_{2}-3}$.

In fact, if $q(f, x, d f)=0, \operatorname{deg} q=l+2$, then

$$
q\left(\frac{x^{n_{1}}}{n_{1}}, x, y ; x^{n_{1}-1}, 0\right) \equiv 0 \quad \bmod \mathcal{O} y^{n_{2}-3} .
$$

Hence

$$
\frac{\partial^{l+2} q}{\partial \hat{\xi}^{l+2}}(0, x, y ; 0,0) \in \mathcal{O} x+\mathcal{O} y^{n_{2}-3} .
$$


Therefore the relation

$$
R_{1}[q] \equiv \frac{n_{1}-1}{2} x^{n_{1}-2} \frac{\partial^{2} q}{\partial \xi^{2}}\left(\frac{x^{n_{1}}}{n_{1}}, x, y ; x^{n_{1}-1}, 0\right) \bmod \mathcal{O} y^{n_{2}-3}
$$

yields the Claim.

Considering both sides of $(20) \bmod \mathcal{O} y^{n_{2}-3}$, we obtain

$$
x^{l\left(n_{1}-1\right)+4 m_{1}} \in \mathcal{O}_{x} x^{(l+1)\left(n_{1}-1\right)} .
$$

This is impossible since $4 m_{1}<n_{1}-1$. Therefore (19) is a counter example against RS 2 (and RS 1 when $l=0$ ).

Quite generally, if $\left(n_{1}, n_{2}, m_{1}\right)$ satisfies

$$
\begin{aligned}
& \frac{n_{1}-1}{k+1} \leq m_{1} \leq \frac{n_{1}-2}{k}, \quad \frac{1}{n_{2}}<\frac{m_{1}}{n_{1}}, \quad n_{2} \geq k+3, \\
& \text { e.g. } \quad n_{1}=k+2, n_{2}=k+3, \quad m_{1}=1,
\end{aligned}
$$

we can prove

$$
l(f) \leq\left[\frac{k}{2}\right]+1, \quad L(f) \leq k
$$

Equalities holds for $k=1,2,3$. See $\S 19$ types $X_{0}, X_{0}^{b}$ and $X_{0}^{p p}$.

\section{§ 8. Generators of $g(s)$ - continued-}

In this section, we study the structure of $g(s)$ when $f$ has an isolated singularity and $L(f)=3$. Our goal is Theorem 2.32. There exist an operator $Q(s)$ in $g(s)$ :

$$
Q(s)=s^{3}+C s^{2}+D s+E,
$$

with $\operatorname{ord}^{T}(Q)=3$.

The case $L(f)=3$ will be divided into two cases: $2=l(f)<L(f)$ $=3$ and $3=l(f)=L(f)$. We call the latter "case $(3,3)$ ". In the former case, there is an operator $P(s)=s^{2}+A s+B$, $\operatorname{ord}^{T}(P)=2$ such that

$$
P(s) f^{s}=a(x) s f^{s-1}
$$

where 


$$
a(x) \notin a+\mathcal{O} f .
$$

We call this case "case $(2,3 ; a)$ ". It is easy to see the following:

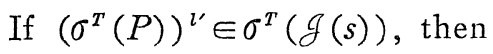

$$
(\mathcal{G}(W[s]))^{l^{\prime}} \subset \sigma^{T}(\mathcal{g}(s)) .
$$

Recall that it was enough to choose $l^{\prime}=2$ in Example 2. 28.

Let $\left(b_{i}(x)\right)$ be a basis of an ideal $\left(\mathfrak{a}^{2}+\mathfrak{a} f\right): f^{2}$. Then, there are operators

$$
B_{j}(s)=b_{j} s^{2}+b_{j}^{\prime} s+b_{j}^{\prime \prime}, \operatorname{ord}^{T}\left(B_{j}\right)=2,
$$

with

$$
B_{j}(s) f^{s}=b_{j}^{\prime \prime \prime}(x) s f^{s-1} .
$$

Here, one may assume that $b_{j}^{\prime \prime \prime} \notin a+O f$ for $j=1, \cdots, J$ and $b_{j}^{\prime \prime \prime}=0$ for $j=J+1, \cdots, J+J^{\prime}$. The following congruence relation can be proved in the same manner as in the proof of Theorem 2.23.

$$
b_{j} s f^{s+1} \equiv b_{j}^{\prime \prime \prime} f^{s} \bmod \mathscr{D}(\mathfrak{a}+\mathcal{O} f) f^{s} .
$$

Let $\mathfrak{c}_{2}$ be the ideal used in Theorem 2.4. Then

$$
c_{2} \subset \sum^{J} \circlearrowleft b_{j}^{\prime \prime \prime}
$$

in view of the structure of $\mathscr{g}(s) \cap(\mathscr{D} s+\mathscr{D})$ and the definition of $\left(b_{j}\right)$. Set

$$
(\mathfrak{a}+\mathcal{O f}): b_{j}^{\prime \prime \prime}=\sum_{k} \mathcal{O} b_{j, k}^{\prime \prime \prime} \quad \text { and } \quad b_{j, k}=b_{j, k}^{\prime \prime \prime} \cdot b_{j} .
$$

(when $j>J, b_{j, k}=b_{j}$ ). Then, we can find operators $B_{j, k}(s)$ in $g(s)$ such that

$$
B_{j, k}(s)=b_{j, k} s^{2}+b_{j, k}^{\prime} s+b_{j, k}^{\prime \prime}, \operatorname{ord}^{T}\left(B_{j, k}\right)=2 .
$$

In general, $g(s) \cap\left(\mathscr{D} s^{2}+\mathscr{D} s+\mathscr{D}\right)$ cannot be generated only by (D)s $+\mathscr{D})(\mathscr{g}(s) \cap(\mathscr{D} s+\mathscr{D}))$ and $\sum \mathscr{D} B_{j, k}$, and we need operators of the following type (cf. Example 2.27):

$$
C_{l}(s)=c_{l}(x, D) s^{2}+c_{l}^{\prime}(x, D) s+c_{l}^{\prime \prime}(x, D),
$$

with $\operatorname{ord}\left(c_{l}\right) \geq 1$.

As is easily seen, we can assume that 


$$
\operatorname{ord}^{T}\left(C_{l}\right)=\operatorname{ord}\left(c_{l}\right)+2=\operatorname{ord}\left(c_{l}^{\prime \prime}\right) .
$$

Moreover, it can be proved by the method employed in $\S 6$ that coefficients of $\sigma\left(c_{l}\right)(x, \xi)$ belong to $\mathfrak{a}: f^{2}$. Set

$$
q(s, x, \xi)=\sigma^{T}(Q)=s^{3}+\left(\sum a_{i} \xi_{i}\right) s^{2}+\left(\sum a_{i j} \xi_{i} \xi_{j}\right) s+\sum a_{i j k} \xi_{i} \xi_{j} \xi_{k} .
$$

Theorem 2.4 shows

$$
R_{1}[q](f, x, d f)=q^{\prime}(f, x, d f),
$$

where

$$
q^{\prime}(s, x, \xi)=\sum b_{i j} \xi_{i} \xi_{j}+\left(\sum b_{i} \xi_{i}\right) s, \text { for some }\left(b_{i j}, b_{i}\right) .
$$

Define the operator $\bar{Q}(s)$ by

$$
\begin{aligned}
\bar{Q}(s)=f s & (s-1)+\left(\sum a_{i}\left(D_{i} f-f_{i}\right)\right)(s-1) \\
& +\sum a_{i j}\left(D_{i} D_{j} f-D_{i} f_{j}-D_{j} f_{i}\right)+\sum a_{i j k} D_{j} D_{k} f_{i} \\
& -\sum b_{i j} D_{j} f_{i}-\sum b_{i} f_{i} .
\end{aligned}
$$

Then, $Q(s) f^{s}=0$ entails

$$
\bar{Q}(s) f^{s}=R(x) f^{s},
$$

where

$$
R(x)=\left(R_{2}[q]-R_{1}\left[\frac{\partial q}{\partial s}\right]-R_{1}\left[q^{\prime}\right]\right)(f, x, d f)
$$

Making use of condition $3^{\circ}$ of Theorem 2.4 and (25), we have

$$
R(x) \equiv 0 \bmod \mathfrak{a}+\mathcal{O} f+\sum \mathcal{O} b_{j}^{\prime \prime \prime},
$$

and hence by (28) and (29),

$$
s^{2} f^{s+1} \equiv b f^{s} \bmod (\mathscr{D} s+\mathscr{D})(\mathfrak{a}+\mathcal{O} f) f^{s},
$$

where

$$
b \in \sum^{J} \mathcal{O} b_{j}^{\prime \prime \prime}
$$

Now we investigate "case $(2,3 ; a) . "$ In this case, $b_{1}=1, J^{\prime}=0$, $b_{1}^{\prime \prime \prime}=a, J=1$. We write $b_{k}\left(b_{k}^{\prime}, b_{k}^{\prime \prime}\right.$ and $B_{k}$, respectively) for $b_{1, k}\left(b_{1, k}^{\prime}, b_{1, k}^{\prime \prime}\right.$ and $B_{1, k}$, respectively), $k=1,2, \cdots, q$. Then we have the following

Proposition 2. 31. In case " $(2,3 ; a)$ ", it holds that 


$$
g(s) \cap\left(\mathscr{D} s^{2}+\mathscr{D} s+\mathscr{D}\right)=\sum_{k=1}^{q} \mathscr{D} B_{k}(s)+\sum_{i=1}^{r} \mathscr{D} A_{i}(s, x, D)+g_{0} .
$$

Proof. Let $T(s)=Q s^{2}+R s+S$ be an element of $g(s)$ with $\operatorname{ord}^{T}(T)=l$. The proof is carried out by induction on $l$. If $l \leq 1$, then the proof is straightforward. Assume $l \geq 2$.

Case i) $\operatorname{ord}(Q)<l-2$. In this case,

$$
\sigma_{l-1}(R) f+\sigma_{l}(S)=0 .
$$

Using the same argument as in Theorem 2.21, we can lower $\operatorname{ord}^{T}(T)$ by subtracting a suitable element in $(\mathscr{D} s+\mathscr{D}) \cap \mathcal{g}(s)$.

Case ii) ord $(Q)=l-2$. In this case,

$$
\{(R-Q A) s+(S-Q B)\} f^{s}=-Q\left(a(x) s f^{s-1}\right) .
$$

Now that the operator in the left-hand side is of order 1 in $s$, and the right-hand side is of order not greater than $l-1$ in $s$, there is an operator $R^{\prime} s+S^{\prime} \in(\mathscr{D} s+\mathscr{D}) \cap g(s)$ such that

$$
\sigma_{l}^{T}\left(R^{\prime} s+S^{\prime}\right)=\sigma_{l}^{T}((R-Q A) s+(S-Q B)) .
$$

Therefore,

$$
\sigma_{l-2}(Q)(x, d f) a(x) \in(\mathfrak{a}+\mathcal{O} f)^{l-1}
$$

by Corollary 2.5. Then, applying the argument of Proposition 2.20, we conclude that all the coefficients of $\sigma_{l-2}(Q)(x, \xi)$ belong to $(\mathfrak{a}+\mathcal{O} f)$ : $a(x)$. Then, choosing appropriate elements $T_{k} \in \mathscr{D}$, we see that $T(s)$ $-\sum T_{k} B_{k}$ is of total order less than $l$ or reduces to an operator discussed in case i).

Q.E.D.

Remark: The proof also shows that

$$
(\mathfrak{a}+\mathcal{O} f): a(x) \supset \mathfrak{a}: f,
$$

if we consider $s A_{i}(s, x, D) \in \mathcal{g}(s)$.

The structure of $\widetilde{M}$ is given by the following theorem. Corresponding theorems for $\mathcal{I}$ and $\mathscr{M}$ are similarly given and we omit them.

Theorem 2. 32. When $L(f)=3$, $\widetilde{\mathscr{M}}$ has a following presentation. 
1) case $(3,3)$.

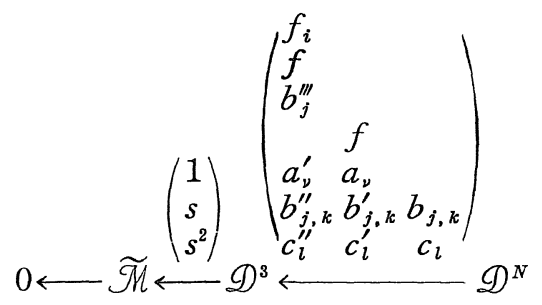

2) case $(2,3 ; a)$.

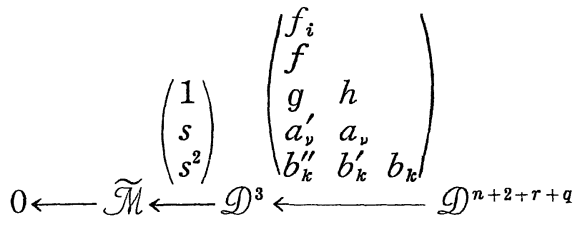

where we can set the row $(g, h, 0)$ either

$$
g=0, h=f \quad \text { or } \quad g=a, h=0 .
$$

In this case, the following inclusion relation holds.

$$
(\mathfrak{a}+\mathcal{O} f): a \supset \mathfrak{a}: f \supset \mathfrak{a}+\mathcal{O} f+\mathcal{O} a
$$

Proof. 1) case $(3,3)$. Suppose

$$
P \overline{1}+Q \bar{s}+R \bar{s}^{2}=0, \text { in } \widetilde{\mathscr{M}} .
$$

As in the first step of the proof of Theorem 3.23, one can assume

$$
\left(P+Q s+R s^{2}\right) f^{s}=\sum R_{i} f_{i} f^{s}+\left(S_{2}+S_{1} s+S_{0} s^{2}\right) f^{s+1},
$$

for some $R_{i}$ and $S_{j} \in \mathscr{D}$. Owing to (30), the right-hand side of (35) can be rewritten as follows:

$$
\sum R_{i}^{\prime} f_{i} f^{s}+\left(S_{2}^{\prime}+S_{1}^{\prime} s\right) f^{s+1}+\sum T_{i} b_{j}^{\prime \prime \prime} f^{s} .
$$

On the other hand, (24) shows

$$
b_{j}^{\prime \prime \prime} \in \mathcal{g}(s)+(\mathscr{D} s+\mathscr{D})(\mathfrak{a}+\mathcal{O} f) .
$$

Thus (32) is proved.

2) case $(2,3 ; a)$. The formula (24) yields

$$
f s \equiv a \bmod g(s)+\mathscr{D}(\mathfrak{a}+\mathcal{O} f) .
$$

Thus Proposition 2.31 and (32) proves (33). 
Next we prove (34). The first inclusion is (31). Using the formula (22) under substitution $s \rightarrow s+1$, we obtain

$$
\left(s^{2}+(A+2) s+(B+A+1)\right) f^{s+1}=a(s+1) f^{s} .
$$

We can eliminate $s^{2} f^{s+1}$ and $s f^{s+1}$ in the left-hand side of (38) by use of (30) and (37). Then we find

$$
(a s+H) f^{s}=0,
$$

for some $H \in \mathscr{D}$ with ord $H \leq 2$. Therefore, Theorem 2.21 (or its proof) proves

$$
a \in \mathfrak{a}: f .
$$

Q.E.D.

We remark that the similar argument of the last part of the proof shows

$$
b_{j}^{\prime \prime \prime} \in \mathfrak{a}: f
$$

for case $(3,3)$.

\section{Chapter III. Determination of $b(s)$}

In this chapter, we explain the method to determine or estimate $b$ functions, and give some explicit formulae in $\S \S 15 \sim 17$. The relation with the local monodromy structure is also discussed.

When we exibit $b$-functions, we sometimes use $p(t)=\frac{1}{2 \pi i} \oint \frac{d}{d s} \log b(s)$ $\times t^{-s} d s$, where the path of integration encounters $R_{f}$ counter clockwise. That is, if $b(s)=\prod_{i=1}^{l}\left(s+\alpha_{i}\right)^{c_{i}}, p(t)=\sum_{i=1}^{l} c_{i} t^{\alpha_{i}}$. Similar notations are used for $\tilde{b}(s)$ and so on.

\section{A. General Procedure}

\section{$\S 9$. Construction of Eigenvectors}

One of the most effective way to seek factors of $b$-functions is the construction of eigenvectors. Let $\mathcal{L}$ be a $\mathscr{D}$-Module and $u$ be its non- 
zero section satisfying

1. There exists $\alpha \in \boldsymbol{C}$ such that

$$
Q(\alpha) u=0 \text { for any } Q(s) \in g(s) .
$$

2. $f u=0$.

Then, $(s-\alpha) \mid b(s)$. In fact, $P(s) f^{s+1}=b(s) f^{s}$ yields $Q(s)=P(s) f$ $-b(s) \in g(s)$. Then $b(\alpha) u=P(\alpha) f u=0$. Hence, $b(\alpha)=0$.

Conditions 1 and 2 assure that the map $\mathscr{M} \rightarrow \mathcal{L}$ defined by $\bar{f}^{s} \mapsto u$ is a well-defined $\mathscr{D}$-homomorphism, and it is an eigenvector belonging to eigenvalue $\alpha$ of the action of $s$ in $\operatorname{Ham}_{\mathscr{D}}(\mathscr{M}, \mathcal{L})$. Since $\mathscr{M}$ is holonomic, if we take a holonomic $\mathcal{L}$, $\operatorname{Hom}_{\mathfrak{D}}(\mathscr{M}, \mathcal{L})$ has finite dimensional stalks [13], and the eigenvalues of $s$ can be calculated. In this course, there follows an important result. The following Theorem 3.3 is due to Theorem 4. 3 [12]. First note the following fact [13].

Proposition 3. 1. Let $X$ be a complex manifold and let $Y$ be its submanifold. Let $\mathcal{L}$ be a holonomic system on $X$. Then

1. There is a regular (in the sense of Whitney) stratification of $X, X=\cup X_{\alpha}$ such that $\breve{S S}(\mathcal{L}) \subset \cup T_{X_{\alpha}}^{*} X$ and Hom ${ }_{\mathscr{D}}\left(\mathcal{L}, \mathscr{B}_{X_{\alpha} \mid X}\right)$ is locally constant sheaf of finite rank. Such stratification is called regular with respect to $\mathcal{L}$.

2. If $\breve{S S}(\mathcal{L}) \subset T_{Y}^{*} X$ and Supp $\mathcal{L} \subset Y, \mathcal{L}$ is locally isomorphic to a finite direct sum of $\mathscr{B}_{Y \mid X}$.

Definition 3.2. Let $X=\cup X_{\alpha}$ be a regular stratification with respect to $\mathcal{M}$. We denote by $b^{i}(s)$ the minimal polynomal of $s$ in $\bigoplus_{\operatorname{codim} X_{\alpha}=i} \operatorname{Hom}_{\mathscr{D}}\left(\mathscr{M}, \mathscr{B}_{X_{\alpha} \mid X}\right)_{x_{\alpha}}, x_{\alpha} \in X_{\alpha} . \tilde{b}^{i}(s)$ is similarly defined for $\widetilde{\mathcal{M}}$.

\section{Theorem 3. 3.}

$$
\begin{aligned}
& \text { 1.c.m. }\left(b^{i}\right)|b| \prod_{i=1}^{n} b^{i}, \\
& \text { 1.c.m. }\left(\tilde{b}^{i}\right)|\tilde{b}| \prod_{i=2}^{n} \tilde{b}^{i} .
\end{aligned}
$$

Proof. It should be noted that $b^{0}=\tilde{b}^{0}=\tilde{b}^{1}=1$ and $b^{1}=s+1$. We 
set $\mathscr{M}^{k}=\prod_{i=0}^{k} b^{i}(s) \cdot \mathscr{M}$, and prove by induction that codim Supp $\mathscr{M}^{k}>k$. Since Supp $\mathscr{M} \subset\{f=0\}$, this is true for $k=0$.

Suppose codim Supp $\mathscr{M}^{k-1}=k$. Let $Y$ be a $k$-codimensional irreducible component of Supp $\mathscr{M}^{k-1}$. Then, there is a non-singular manifold $Y^{\prime} \subset Y$ such that $\operatorname{codim}\left(Y-Y^{\prime}\right)>k$ and $\breve{S} S\left(\mathscr{M}^{k-1}\right) \cap T_{*} X \times Y_{X}^{\prime} \subset T_{Y^{\prime}}^{*} X$. Therefore $\mathscr{M}^{k-1} \simeq\left(\mathscr{B}_{Y^{\prime} \mid X}\right)^{N}$ by 2 of Proposition 3.1. Since $b^{k}(s)$ Hom $(\mathscr{M}$, $\left.\mathscr{B}_{Y^{\prime} \mid X}\right)_{y^{\prime}}=0, b^{k}(s)$ Ham $_{\mathscr{D}}\left(\mathscr{M}, \mathscr{M}^{k-1}\right)_{y^{\prime}}=0$. Then the commutative diagram

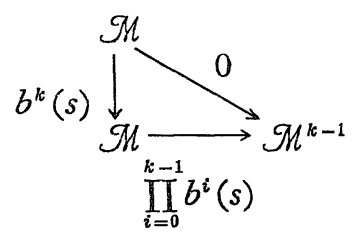

shows $\mathscr{M}_{y^{\prime}}^{k}=0$. That is, codim Supp $\mathscr{M}^{k}>k$. Lastly, we obtain $\mathscr{M}^{n}$ $=\prod_{i=0}^{n} b^{i}(s) \cdot \mathcal{M}=0$.

Therefore $b(s) \mid \prod_{i=0}^{n} b^{i}(s)$. 1.c.m. $\left(b^{i}\right) \mid b$ was proved in the arguments at the beginning of this section. The proof of (2) is almost the same.

Q.E.D.

As a special case, if $f$ has an isolated singularity, $b^{n}(s)$ turns out to be the minimal polynomial of $s$ in $\widetilde{\mathcal{M}}$, and hence $b(s)=(s+1) b^{n}(s)$. Thus the determination of $b(s)$ is reduced to the study of $\widetilde{M}$ in case of isolated singularity.

At this stage, we note a simple but useful proposition.

Proposition 3.4. Assume $f$ is a weighted homogeneous polynomial with $X_{0}=\sum a_{i} x_{i} D_{i}$ (isolated singularity or not) such that for a polynomial $p(x) \in \mathbb{C}[x],\left(X_{0}+\sum a_{i}\right) p(x)=0$ yields $p(x)=0$. Then,

$$
\operatorname{Ham}_{\mathscr{D}}\left(\widetilde{M}, \mathscr{B}_{p t}\right) \underset{\rightarrow}{\operatorname{Ham}}\left(\mathcal{M}, \mathscr{B}_{p t}\right) \text {. }
$$

Proof. We have only to prove,

"If $\Delta \in \mathscr{B}_{p t}$ satisfies $X_{i j} \Delta=0$ and $f \Delta=0$, then $f_{i} \Delta=0$."

We set $\Delta=q(D) \delta(x)$. Since $\xi_{j} f_{i}\left(-D_{\xi}\right) q=\xi_{i} f_{j}\left(-D_{\xi}\right) q$, there is an $r(\xi)$ such that $\int_{i}\left(-D_{\xi}\right) q(\xi)=\xi_{i} r(\xi)$. Using the condition $f \Delta=0$, 


$$
\begin{aligned}
0 & =\sum a_{i} D_{\xi_{i}} f_{i}\left(-D_{\xi}\right) q(\xi) \\
& =\sum a_{i} D_{\xi_{i}} \xi_{i} r(\xi) \\
& =\left(\sum a_{i} \xi_{i} D_{\xi_{i}}+\sum a_{i}\right) r(\xi) .
\end{aligned}
$$

By the condition on $X_{0}$, we can conclude that $r(\xi)=0$. Q.E.D.

M. Kashiwara conjectured that $b(s)=\prod b^{i}(s)$ ([12]). This, however, fails to hold in general, as seen in the following.

Example 3. 5. $f=x^{3}+y^{3} z$ (cf. Example 4. 20).

$g(s)$ is generated by $y D_{y}-3 z D_{z}, y^{3} D_{x}-3 x^{2} D_{z}, y^{2} z D_{x}-x^{2} D_{y}$ and $s-\frac{1}{3}\left(x D_{x}+y D_{y}\right)$. The stratification is $X=\cup X_{a}, X_{0}=(X \backslash\{f=0\})$, $X_{1}=\{f=0\} \backslash\{x=y=0\}, X_{2}=\{x=y=0\} \backslash\{0\}, X_{3}=\{0\} . \breve{S S}(\mathscr{M})=T_{X_{1}}^{*} X$ $\cup T_{X_{2}}^{*} X \cup T_{X_{3}}^{*} X$.

$$
b^{1}(s)=s+1 . \quad b^{2}(s)=\prod_{\nu=2}^{4}\left(s+\frac{\nu}{3}\right) . \quad b^{3}(s)=\left(s+\frac{4}{3}\right)\left(s+\frac{5}{3}\right) .
$$

$b^{2}(s)$ can be calculated by the construction of eigenvectors $z^{-(k+1) / 3} D_{x}^{h} D_{y}^{k}$ $\delta(x, y)$ belonging to $-(h+k+2) / 3, h, k=0,1 . b^{3}(s)$ is known by eigenvectors $D_{x}^{h} D_{y}^{2} \delta(x, y, z), h=0,1$, which belong to $-4 / 3$ and $-5 / 3$. On the other hand, explicit calculation of $P(s)$ (cf. $\S 11$ ) shows

$$
P(s) f^{s+1}=\prod_{\nu=2}^{5}\left(s+\frac{\nu}{3}\right) f^{s},
$$

where

$$
\begin{gathered}
P(s)=D_{x} D_{y}^{2} Q+\frac{1}{3}\left(s+\frac{5}{3}\right)\left(\frac{2}{3} D_{x}^{3} D_{y} y+D_{y}^{3} D_{z}\right)+\left(s+\frac{4}{3}\right)\left(s+\frac{5}{3}\right) D_{x}^{2}, \\
Q=\frac{1}{3}\left(\frac{1}{3} D_{x}^{2} y^{2}+x D_{y} D_{z}\right) .
\end{gathered}
$$

Thus $b(s)=\prod_{\nu=2}^{\dot{j}}\left(s+\frac{\nu}{3}\right)$. In this case, both $b^{2}(s)$ and $b^{3}(s)$ have a factor $\left(s+\frac{4}{3}\right)$, but $b(s)$ has $\left(s+\frac{4}{3}\right)$ as a simple factor.

\section{$\S 10$. Construction of Operators}

If we can find $P(s) \in \mathscr{D}[s]$ such that 


$$
P(s) f^{s+1}=b^{\prime}(s) f^{s},
$$

$b(s)$ is a divisor of $b^{\prime}(s)$. There is a systematic method to construct such $P(s)$ when $f$ is a weighted homogeneous polynomial. This procedure was pointed out by $M$. Sato at the early stage of the theory of $b$-functions. For the simplicity of explanation, let us assume that $f(x, y)$ is a weighted homogeneous polynomial of 2 -rariables: $X_{0} f=f, X_{0}=\alpha x D_{x}$ $+\beta y D_{y}$.

Suppose one can find operators $A(s)$ and $B(s)$ such that

$$
\begin{aligned}
& A(s) f^{s+1}=a(s) x^{i+1} y^{j} f^{s}, \\
& B(s) f^{s+1}=a(s) x^{i} y^{j+1} f^{s} .
\end{aligned}
$$

The11,

$$
\begin{aligned}
\left(\alpha D_{x} A+\beta D_{y} B\right) f^{s+1} & =a(s)\left(\alpha D_{x} x+\beta D_{y} y\right)\left(x^{i} y^{j} f^{s}\right) \\
& =a(s)(s+(i+1) \alpha+(j+1) \beta) x^{i} y^{j} f^{s} .
\end{aligned}
$$

This process shows that if one has equalities

$$
A_{i}(s) f^{s+1}=a_{i}(s) x^{i} y^{m-i} f^{s} \quad i=0,1, \cdots, m,
$$

then one can construct a $P(s) \in \mathscr{D}[s]$ such that (3) holds, and each roots of $b^{\prime}(s)$ is that of 1.c.m. $\left(a_{i}(s)\right)$ or of the form $-(\alpha k+\beta l) 1 \leq k, l$, $k+l \leq m+1$. More generally:

Proposion 3.6. i) Let $f\left(x_{1}, \cdots, x_{k}, y_{1}, \cdots, y_{l}\right)$ be a polynomial satisfying 1. $X_{0} f=f$ for $X_{0}=\sum_{i=1}^{k} a_{i} x_{i} D_{i} a_{i} \in Q, 2$. For all multiindices $\alpha,|\alpha|=m$, there exist $P_{\alpha}(s)$ such that

$$
P_{\alpha}(s) f^{s+1}=a_{\alpha}(s) x^{\alpha} f^{s}, a_{\alpha}(s) \in \mathbb{C}[s] .
$$

Then,

$$
b(s) \mid \prod_{n=k}^{m+k-1} 1 . \text { c.m. }\left(s+\sum a_{i} \nu_{i}\right) \cdot \text { l.c.m. }\left(a_{\alpha}(s)\right) .
$$

Assume further that $\operatorname{ord}^{T} P_{\alpha}(s)=\operatorname{deg} a_{\alpha}(s)$ in (4). Then, we can find $P(s)$ and $b^{\prime}(s)$ in (3) such that $\operatorname{ord}^{T} P(s)=\operatorname{deg} b^{\prime}(s)$.

ii) When $f(x)$ is a reighted homogeneous polynomial with weight $\left(a_{1}, \cdots, a_{n}\right)$ and of isolated singularity, 


$$
b_{f}(s) \mid(s+1) \prod_{h=n}^{\mu+n-1} 1 . c . m .\left(s+\sum a_{i} \nu_{i}\right)
$$

where $\mu=\operatorname{dim} \mathcal{O} / \mathfrak{a}$.

Proof. Relation (5) can be proved analogously as preceding arguments. Last part of i) is obvious by the very construction of $P(s)$. When $f$ has an isolated singularity, $\mathfrak{a} \supseteq \mathfrak{m}^{\mu}, \mu=\operatorname{dim} \mathcal{O} / \mathfrak{a}$. Therefore, (4) holds for any $\alpha,|\alpha|=\mu$ with $a_{\alpha}(s)=s+1$.

Q.E.D.

In the next section, we give the explicit formula for $b_{f}$ in case ii). We present (6) here simply to show that our elementary procedure even proves the existence of $b_{f}$ for some polynomials.

The estimate (5) is not the best possible one in general.

Example 3. 7. $f=x^{n}+y z^{m}$.

$$
\begin{aligned}
& \frac{1}{n} D_{x} f^{s+1}=(s+1) x^{n-1} f^{s} \\
& D_{y} f^{s+1}=(s+1) z^{m} f^{s} .
\end{aligned}
$$

Then,

$$
\left(z^{m-1}\left(\frac{D_{x}}{n}\right)^{2}+\frac{1}{m} x^{n-2} D_{y} D_{z}\right) f^{s+1}=(s+1)\left(s+1+\frac{n-1}{n}\right) x^{n-2} z^{m-1} f^{s+1} .
$$

Thus, all calculations are carried out about the monomials $x^{i} y^{j}$ $0 \leq i \leq n-1,0 \leq j \leq m$. And we have

$$
b(s) \mid(s+1) \underset{\substack{1 . c . m . \\ 1 \leq k \leq n-1 \\ 1 \leq l \leq m}}{\operatorname{lin}}\left(s+\frac{k}{n}+\frac{l}{m}\right) .
$$

Since $\delta(f), y^{-1 / m} D_{x}^{k-1} D_{z}^{l-1} \delta(x, z)$ and $D_{x}^{k-1} D_{z}^{m-1} \delta(x, y, z)$ belong to eigenvalues $-1,-\left(\frac{k}{n}+\frac{l}{m}\right)$ and $-\left(\frac{k}{n}+1\right)$, this is the equality.

Example of this type can be found in $\S 21$.

\section{B. Isolated Singularities}




\section{$\S 11$. Quasi-Homogeneous Isolated Singularities}

Let $f$ be a quasi-homogeneous analytic function with isolated singularity at $0 \in C^{n}$. In this case, a result of K. Saito [22] tells us that we can find a suitable coördinate transformation so that $X_{0} f=f$ with $X_{0}=\sum_{i=1}^{n} a_{i} x_{i} D_{i}, a_{i} \in Q^{+}$. We shall show that $b$ is determined by $\left(a_{1}, \cdots, a_{n}\right)$.

Applying the functors $\operatorname{Ham}_{\mathscr{D}}\left(\cdot, \mathscr{B}_{p t}\right)$ and $\Omega^{n} \otimes \cdot$ to the presentation II (10) of $\widetilde{\mathscr{M}}$, there are two exact sequences.

$$
\begin{aligned}
& 0 \rightarrow F \rightarrow \mathscr{B}_{p t} \rightarrow \mathscr{B}_{p t}^{n}, \\
& 0 \leftarrow F^{*} \leftarrow \Omega^{n} \leftarrow\left(\Omega^{n}\right)^{n} .
\end{aligned}
$$

Here,

$$
\begin{aligned}
& F=\operatorname{Ham}_{\mathscr{D}}\left(\widetilde{M}, \mathscr{B}_{p t}\right)=\left\{\Delta(x) \in \mathcal{B}_{p t} \mid f_{i}(x) \Delta(x)=0 \forall i\right\} \\
& F^{*}=\Omega^{n} \otimes_{\mathscr{D}} \widetilde{\mathscr{H}}=\Omega^{n} / \mathfrak{a} \Omega^{n} \simeq \mathcal{O} / \mathfrak{a},
\end{aligned}
$$

and they are dual to each other.

The action of $s$ in $F$ is $X_{0}$, and that in $O / \mathfrak{a}$ is $X_{0}^{*}=-X_{0}-\sum a_{i}$.

Since we can take monomials as a basis of $\mathcal{O} / \mathfrak{a}, s$ is diagonalizable. The following theorem was proved by Kashiwara-Sato-Miwa [19]. Here, we give a simple proof of it.

As we have shown in $\S 9, b(s)=(s+1) \tilde{b}(s)$, where $\tilde{b}(s)$ is a minimal polynomial of $s$ in $\operatorname{Hom}_{\mathscr{D}}\left(\widetilde{\mathcal{M}}, \mathscr{B}_{p t}\right)$. We denote by $\widetilde{B}(s)$ the characteristic polynomial of $s$, and by $\widetilde{P}(t)$, the associated trace-type formula.

Theorem 3.8. $\widetilde{P}(t)=\prod \frac{t^{a_{i}}-t}{1-t^{a_{i}}}$.

Proof. Let $\left\{m_{i}\right\} \quad 1 \leq i \leq \mu$ be monomials such that $\left\{m_{i} \bmod a\right\}$ generates $O / a$.

Claim:

$$
\mathbb{C}\left[x_{1}, \cdots, x_{n}\right] \simeq \mathbb{C}\left[f_{1}, \cdots, f_{n}\right] \otimes \sum_{i=1}^{\mu} \mathbb{C} m_{i} .
$$

In fact, since $\left(f_{1}, \cdots, f_{n}\right)$ is algebraically independent, $C\left[f_{1}, \cdots, f_{n}\right]$ is 
isomorphic to a polynomial ring. One can define the map from the right to the left-hand side naturally. The inverse can be constructed as follows. Take $\varphi(x) \in \boldsymbol{C}\left[x_{1}, \cdots, x_{n}\right]$. Then, there are unique $a_{i} \in \boldsymbol{C}$ such that $\varphi(x)=\sum a_{i} m_{i}+\sum b_{i} f_{i}$, since $\mathcal{O} / \mathfrak{a}$ is a rector space. We can rewrite further

$$
\sum_{i} b_{i} f_{i}=\sum_{i}\left(\sum_{j} a_{i j} m_{j}+b_{i j} f_{j}\right) f_{i}
$$

and $a_{i j}$ are uniquely determined since $\mathfrak{a} / \mathfrak{a}^{2} \simeq(\mathcal{O} / \mathfrak{a}[T])^{(1)}$ by Lemma 2. 20. Here, $(\mathcal{O} / \mathfrak{a}[T])^{(\nu)}$ denotes the $\nu$-th homogeneous part of $\mathcal{O} / \mathfrak{a}[T]$. Using $\mathfrak{a}^{\nu} / \mathfrak{a}^{\nu+1} \simeq(\Theta / \mathfrak{a}[T])^{(\nu)}$, we can proceed further and find the unique $\left(a_{i}, a_{i j}, a_{i j k}, \cdots\right)$ such that

$$
\varphi(x)=\sum a_{i} m_{i}+\sum a_{i j} m_{i} f_{j}+\sum a_{i j k} m_{i} f_{j} f_{k}+\cdots
$$

When the weight of $\varphi$ is $d$, this series terminates at most $[2 d]+1$ terms.

Consider the Poincaré polynomials of both sides of (7). Then.

$$
\Pi\left(1-t^{a_{i}}\right)^{-1}=\Pi\left(1-t^{1-a_{i}}\right)^{-1} \times q(t) .
$$

$q(t)$ is nothing but $\operatorname{tr}\left(t^{X_{0}}: \mathcal{O} / \mathfrak{a}\right)$. Therefore,

$$
\begin{aligned}
\operatorname{tr}\left(t^{-s}: \mathcal{O} / \mathfrak{a}\right) & =\operatorname{tr}\left(t^{X_{0}+\Sigma a_{i}}: \mathcal{O} / \mathfrak{a}\right) \\
& =\Pi t^{a_{i}} \Pi \frac{1-t^{1-a_{i}}}{1-t^{a_{i}}} \\
& =\Pi \frac{t^{a_{i}}-t}{1-t^{a_{i}}} .
\end{aligned}
$$

Corollary 3.9. i) $\operatorname{dim} F=\operatorname{dim} F^{*}=\mu=\Pi \frac{1-a_{i}}{a_{i}}$.

ii) Let $\widetilde{P}(t)=\sum_{a \in \mathbb{Q}_{+}} q_{\alpha} t^{\alpha}$ be the expansion into the polynomial with fractional power. Then,

$$
b(s)=(s+1) \prod_{q_{\alpha} \neq 0}(s+\alpha) .
$$

iii) $\quad \tilde{b}(-n-s)=(-)^{\tilde{a}} \tilde{b}(s), \quad \tilde{d}=\operatorname{deg} \tilde{b}$.

The proof is obvious.

We remark that the eigenvector belonging to $-\sum a_{i}$ is 1 in $O / a$ and $\delta(x)$ in $F$, and that to $-\left(n-\sum a_{i}\right)$ is $\operatorname{Hess}(f)$ in $O / \mathfrak{a}$. 
Since $\widetilde{M} \simeq \mathscr{D} / \mathscr{D} \mathfrak{a}$, one can rewrite

$$
\tilde{b}\left(X_{0}\right)=\sum p_{i}(x, D) f_{i} .
$$

Then, defining $P(x, D)=\sum p_{i}(x, D) D_{i}$, we obtain

$$
\begin{aligned}
P(x, D) f^{s+1} & =(s+1) \sum p_{i}(x, D) f_{i} f^{s}, \\
& =(s+1) \tilde{b}\left(X_{0}\right) f^{s}, \\
& =b(s) f^{s} .
\end{aligned}
$$

Put $\tilde{d}=\operatorname{deg} \tilde{b}$. If $d=\max \left(\operatorname{ord} p_{i}\right)>\tilde{d}$, then $\sum \sigma_{d}\left(p_{i}\right) f_{i}=0$. Therefore, by setting $p_{i}(x, D)=\sum D^{\alpha} a_{i, \alpha}(x)$ for $\sigma_{d}\left(p_{i}\right)=\sum a_{i, \alpha}(x) \xi^{\alpha}$, we obtain $\sum p_{i}^{\prime}(x, D) f_{i}=0$. Then

$$
\tilde{b}\left(X_{0}\right)=\sum\left(p_{i}-p_{i}^{\prime}\right) f_{i}, \operatorname{ord}\left(p_{i}-p_{i}^{\prime}\right)<\operatorname{ord} p_{i} .
$$

Therefore, we can choose $p_{i}{ }^{\prime}$ s such that ord $p_{i} \leq \operatorname{deg} \tilde{b}$ and at least one of them is an equality. Then

$$
\text { ord } P=\max \left(\operatorname{ord} p_{i}+1\right)=\operatorname{deg} b .
$$

Thus we again find Cor. 2.26. For example, when $f=\sum x_{i}^{2}, \tilde{b}(s)$ $=s+\frac{n}{2}$.

$$
\tilde{b}\left(X_{0}\right)=\frac{1}{2} \sum x_{i} D_{i}+\frac{n}{2}=\frac{1}{2} \sum D_{i} x_{i}=\frac{1}{4} \sum D_{i} f_{i} .
$$

Therefore $P(x, D)=\frac{1}{4} \sum D_{i}^{2}=\frac{1}{4} \Delta$.

\section{§12. $L(f)=2$}

We use the presentation II (13). Applying the functor $\operatorname{Ham}_{\mathscr{D}}\left(\cdot, \mathscr{B}_{p t}\right)$

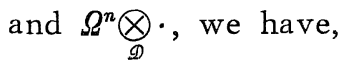

$$
\begin{aligned}
& 0 \rightarrow F \rightarrow \mathcal{B}_{p t}^{2} \rightarrow \mathcal{B}_{p t}^{n+r+1}, \\
& 0 \leftarrow F^{*} \leftarrow\left(\Omega^{n}\right)^{2} \leftarrow\left(\Omega^{n}\right)^{n+r+1} .
\end{aligned}
$$

Here

$$
\begin{aligned}
F & =\operatorname{Ham}_{\mathscr{D}}\left(\widetilde{\mathscr{M}}, \mathscr{B}_{p t}\right) \\
& =\left\{\left(\begin{array}{l}
u \\
v
\end{array}\right) \in \mathscr{B}_{p t}^{2} \mid u \in F_{1}, a_{\nu}(x) v+a_{\nu}^{\prime}(x, D) u=0,1 \leq \nu \leq r\right\},
\end{aligned}
$$




$$
F_{1}=\left\{u \in \mathscr{B}_{p t} \mid(\mathfrak{a}+\mathcal{O} f) u=0\right\} .
$$

The sequence (10) will be analysed later.

Set $F_{2}=\left\{v \in \mathscr{B}_{p t} \mid(\mathfrak{a}: f) v=0\right\}$. Since $L(f)=2$, we have $f^{2} \in \mathfrak{a} f$ $+\mathfrak{a}^{2}$, and hence $F_{2} \subset F_{1}$. Set $\mu_{1}=\operatorname{dim} F_{1}=\operatorname{dim} \mathcal{O} /(\mathfrak{a}+\mathcal{O} f), \mu_{2}=\operatorname{dim} F_{2}$ $=\operatorname{dim} \mathcal{O} /(\mathfrak{a}: f)$. Then, $\mu_{1}+\mu_{2}=\mu=\operatorname{dim} \Theta / \mathfrak{a}$, since

$$
\operatorname{Coker}(\mathcal{O} \rightarrow \mathcal{O} / \mathfrak{a})=\mathcal{O} /(\mathfrak{a}+\mathcal{O} f)
$$

and

$$
\operatorname{Coim}(\mathcal{O} \rightarrow \mathcal{O} / \mathfrak{a})=O /(\mathfrak{a}: f) .
$$

We choose a basis of $F_{2}$ and $F_{1}$ such that $\left(u_{1}, \cdots, u_{\mu_{2}}\right)$ is a basis of $F_{2}$ and $\left(u_{1}, \cdots, u_{\mu_{2}}, u_{\mu_{2}+1}, \cdots, u_{\mu_{1}}\right)$ is one of $F_{1}$. If $\sum e_{\nu}(x, D) a_{\nu}(x)=0$,

$$
\begin{aligned}
& \left(\sum e_{\nu}(x, D) a_{\nu}^{\prime}(x, D)\right) f^{s} \\
& \quad=-s\left(\sum e_{\nu}(x, D) a_{\nu}(x)\right) f^{s}=0 .
\end{aligned}
$$

Therefore $\sum e_{\nu}(x, D) a_{\nu}^{\prime}(x, D) \in \mathscr{g}_{0} \subset \mathscr{D} \mathfrak{a}$. Then we can solve the system of equations for $v_{i}, 1 \leq i \leq \mu_{1}$,

$$
a_{\nu}(x) v_{i}=-a_{\nu}^{\prime}(x, D) u_{i} \quad 1 \leq \nu \leq r,
$$

and $v_{i}$ 's are determined $\bmod F_{2}$. Thus, $\left(\begin{array}{c}0 \\ u_{i}\end{array}\right), 1 \leq i \leq \mu_{2}$ and $\left(\begin{array}{c}u_{i} \\ v_{i}\end{array}\right), 1 \leq i \leq \mu_{1}$ forms a basis of $F$.

This can be summarized in

Theorem 3.9. Let $f$ be a holomorphic function having isolated singularity and of $L(f)=2$. Then $\operatorname{Hom}_{\mathscr{D}}\left(\widetilde{\mathcal{M}}, \mathscr{B}_{p t}\right)$ is u-dimensional and its basis is given by the form

$$
\left\{\left(\begin{array}{l}
0 \\
u_{1}
\end{array}\right), \cdots,\left(\begin{array}{c}
0 \\
u_{\mu_{2}}
\end{array}\right),\left(\begin{array}{l}
u_{1} \\
v_{1}
\end{array}\right), \cdots,\left(\begin{array}{l}
u_{\mu_{1}} \\
v_{\mu_{1}}
\end{array}\right)\right\}
$$

where $\left(u_{1}, \cdots, u_{\mu_{2}}\right)$ forms a basis of $\left\{u \in \mathscr{B}_{p t} \mid(\mathfrak{a}: f) u=0\right\},\left(u_{1}, \cdots, u_{\mu_{2}}\right.$, $\left.u_{\mu_{2}+1}, \cdots, u_{\mu_{1}}\right)$ forms one of $\left\{u \in \mathscr{B}_{p t} \mid(\mathfrak{a}+\mathcal{O} f) u=0\right\}$ and $v_{i}$ satisfy equations

$$
a_{\nu}(x) v_{i}=-a_{\nu}^{\prime}(x, D) u_{i} \quad 1 \leq \nu \leq r
$$

$\tilde{b}(s)$ can be calculated as a minimal polynomial of 


$$
s:\left(\begin{array}{l}
u \\
v
\end{array}\right) \mapsto\left(\begin{array}{rr}
0 & 1 \\
-B & -A
\end{array}\right)\left(\begin{array}{l}
u \\
v
\end{array}\right) \quad \text { in } F
$$

Let $b_{i j}$ be the components of a matrix

$$
\tilde{b}\left(\left(\begin{array}{cr}
0 & 1 \\
-B & -A
\end{array}\right)\right)=\left(\begin{array}{ll}
b_{11} & b_{12} \\
b_{21} & b_{22}
\end{array}\right) .
$$

We have $\left(b_{11}, b_{12}\right)\left(\begin{array}{c}f^{s} \\ s f^{s}\end{array}\right)=\tilde{b}(s) f^{s}$, and

$$
\left(b_{11}, b_{12}\right)=\sum\left(c^{i}, 0\right) f_{i}+(c, 0) f+\sum d_{\nu}\left(a_{\nu}^{\prime}(x, D), a_{\nu}(x)\right),
$$

by the presentation of $\widetilde{\mathscr{M}}$ and the definition of $\tilde{b}(s)$. Set

$$
P(s, x, D)=\sum c^{i} D_{i}+(s+1) \cdot c .
$$

Then, $P(s, x, D) f^{s+1}=b(s) f^{s}$. This construction of $P$ is applicable even if $L(f) \geq 3$, when $g(s)$ is determined and $\tilde{b}(s)$ is known.

We note that we can take $P(s)$ such that $\operatorname{ord}^{T} P=\operatorname{deg} b$ (Corollary 2. 26). This can be seen directly as follows. By the definition of $b_{i j}$, ord $b_{11} \leq \tilde{d}$, and ord $b_{12} \leq \tilde{d}-1$, where $\tilde{d}=\operatorname{deg} \tilde{b}$. Owing to the relation $b_{12}=\sum d_{\nu} a_{\nu}(x)$, we can choose ord $d_{\nu} \leq \tilde{d}-1$, by the same reasoning as in p. 161. Then, ord $\left(b_{11}-\sum d_{\nu} a_{\nu}(x, D)\right) \leq \tilde{d}$ and hence we can take $\max \left(\operatorname{ord} c^{i}\right.$, ord $\left.c\right) \leq \tilde{d}$ owing to the relation

$$
b_{11}-\sum d_{\nu} a_{\nu}(x, D)=\sum c^{i} f_{i}+c f .
$$

Therefore, $\operatorname{ord}^{T} P(s) \leq \tilde{d}+1=\operatorname{deg} b$, and since the converse inequality is obvious, this must be an equality.

Let $u=\left(\begin{array}{l}\Delta \\ \Delta^{\prime}\end{array}\right) \in \mathscr{B}_{p t}^{2}$.

Then $u$ satisfies $\left(\begin{array}{cc}0 & 1 \\ -B & -A\end{array}\right) u=\alpha u$, and $u \in F$ if and only if $\Delta$ satisfies

$$
\begin{aligned}
& \Delta^{\prime}=\alpha \Delta, f \Delta=f_{i} \Delta=0, \\
& A_{\nu}(\alpha, x, D) \Delta=0, \\
& \left(\alpha^{2}+A \alpha+B\right) \Delta=0 .
\end{aligned}
$$

Therefore, we sometimes call $\Delta$ itself, instead of $u=\left(\begin{array}{c}\Delta \\ \alpha \Delta\end{array}\right)$ an eigenvector. 
Next, let $u^{\prime}=\left(\begin{array}{c}\Delta^{\prime} \\ \Delta^{\prime \prime}\end{array}\right)$ and $u=\left(\begin{array}{c}\Delta \\ \alpha \Delta\end{array}\right)$ be root vectors belonging to an eigenvalue $\alpha$ :

$$
s \cdot\left(u, u^{\prime}\right)=\left(u, u^{\prime}\right)\left(\begin{array}{rr}
\alpha & 1 \\
\alpha
\end{array}\right)
$$

Then

$$
\begin{aligned}
& \Delta^{\prime \prime}=\alpha \Delta^{\prime}+\Delta, f \Delta^{\prime}=f_{i} \Delta^{\prime}=0, \\
& A_{\nu}(\alpha, x, D) \Delta^{\prime}=-a_{\nu}(x) \Delta, \\
& \left(\alpha^{2}+A \alpha+B\right) \Delta^{\prime}=-(2 \alpha+A) \Delta .
\end{aligned}
$$

Conversely, if $\Delta$ is an eigenfunction and $\Delta^{\prime}, \Delta^{\prime \prime}$ satisfy above formulae, $u$ and $u^{\prime}$ are root vectors: Especially when

$$
(a: f) \Delta=0
$$

and

$$
(2 \alpha+A) \Delta=0,
$$

$u=\left(\begin{array}{c}\Delta \\ \alpha \Delta\end{array}\right)$ and $u^{\prime}=\left(\begin{array}{l}0 \\ \Delta\end{array}\right)$ form those belonging to an eigenvalue $\alpha$.

We can also use II (32). In this case, the isomorphism holds

$$
F^{*} \simeq(\mathcal{O} /(\mathfrak{a}+\mathcal{O} f))\left(\begin{array}{l}
1 \\
0
\end{array}\right) \oplus(\mathcal{O} / \mathfrak{a}: f)\left(\begin{array}{l}
0 \\
1
\end{array}\right),
$$

as a vector space. The action of $s$ is, under the above isomorphism,

$$
s:\left(\begin{array}{l}
q \\
r
\end{array}\right) \mapsto\left(\begin{array}{cc}
0 & -B^{*} \\
1 & -A^{*}
\end{array}\right)\left(\begin{array}{l}
q \\
r
\end{array}\right) .
$$

\section{§ 13. $L(f)=3$}

First, we consider case $(2,3 ; a)$. Define the spaces of $\delta$-functions $F_{i}$ as follows:

$$
\begin{aligned}
& F_{1}=\left\{u \in \mathscr{B}_{p t} \mid(\mathfrak{a}+\mathcal{O} f+\mathcal{O} a) u=0\right\}, \\
& F_{2}=\left\{u \in \mathscr{B}_{p t} \mid(\mathfrak{a}: f) u=0\right\}, \\
& F_{3}=\left\{u \in \mathcal{B}_{p t} \mid((\mathfrak{a}+\mathcal{O} f): a) u=0\right\} .
\end{aligned}
$$

The relation II (34) shows there are canonical inclusions: 


$$
F_{3} \subset F_{2} \subset F_{1} \text {. }
$$

Since $\operatorname{dim} F_{1}+\operatorname{dim} F_{3}=\operatorname{dim} \mathcal{O} /(\mathfrak{a}+\mathcal{O} f)$, we have

$$
\sum_{i=1}^{3} \operatorname{dim} F_{1}=\operatorname{dim} \mathcal{O} / \mathfrak{a}=\mu .
$$

Using the presentation II (33) with $g=a$ and $h=0$, we obtain the following theorem. The method of proof is similar to that of Theorem 3.9 and we omit it.

Theorem 3.10. Let $f$ be a holomorphic function having isolated singularity and $2=l(f)<L(f)=3$. Then Hom $\left(\widetilde{\mathscr{A}}, \mathscr{B}_{p t}\right)$ is $\mu$-dimensional and its basis is given by the form

$$
\left\{\left(\begin{array}{l}
0 \\
0 \\
u_{1}
\end{array}\right) \cdots\left(\begin{array}{l}
0 \\
0 \\
u_{\mu_{3}}
\end{array}\right),\left(\begin{array}{c}
0 \\
u_{1} \\
v_{1}^{\prime}
\end{array}\right), \cdots,\left(\begin{array}{c}
0 \\
u_{\mu_{2}} \\
v_{\mu_{2}}^{\prime}
\end{array}\right), \cdots,\left(\begin{array}{c}
u_{1} \\
v_{1} \\
w_{1}
\end{array}\right), \cdots,\left(\begin{array}{c}
u_{\mu_{1}} \\
v_{\mu_{1}} \\
w_{\mu_{1}}
\end{array}\right)\right\} .
$$

where $\left(u_{1}, \cdots, u_{\mu_{1}}\right)$ is a basis of $F_{1}$ for $i=1,2,3$ and $\left(u, v, v^{\prime} w\right)$ satisfy the following equations:

$$
\begin{aligned}
& b_{k} v_{i}^{\prime}+b_{k}^{\prime} u_{i}=0, \\
& a_{\nu} v_{i}+a_{\nu}^{\prime} u_{i}=0, \\
& b_{k} w_{i}+b_{k}^{\prime} v_{i}+b_{k}^{\prime \prime} u_{i}=0 .
\end{aligned}
$$

The action of $s$ in $F=\operatorname{Ham}_{\mathscr{D}}\left(\widetilde{\mathscr{M}}, \mathscr{B}_{p t}\right)$ is given by

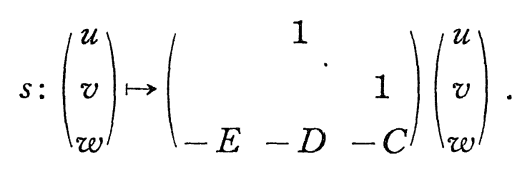

Let $c_{i j}$ be the components of a matrix

$$
\tilde{b}\left(\left(\begin{array}{ccc}
1 & \\
& & 1 \\
-E & -D & -C
\end{array}\right)\right)=\left(\begin{array}{lll}
c_{11} & c_{12} & c_{13} \\
c_{21} & c_{22} & c_{23} \\
c_{31} & c_{32} & c_{33}
\end{array}\right) .
$$

The presentation II (33) with $g=0, h=f$ gives

$$
\begin{aligned}
\left(c_{11}, c_{12}, c_{13}\right)= & \sum\left(c^{i}, 0,0\right) f_{i}+\left(c_{1}, c_{2}, 0\right) f+\sum d_{\nu}\left(a_{\nu}^{\prime}, a_{\nu}, 0\right) \\
& +\sum e_{k}\left(b_{k}^{\prime \prime}, b_{k}^{\prime}, b_{k}\right) .
\end{aligned}
$$


Define

$$
P(s)=\sum c^{i} D_{i}+(s+1) c_{1}+s(s+1) c_{2} .
$$

Then

$$
P(s) f^{s+1}=b(s) f^{s} .
$$

We remark that this $b$-operator $P(s)$ can be so chosen that

$$
\operatorname{ord}^{T}(P)=\operatorname{deg} b \text {. }
$$

It should be noted that we cannot apply Proposition 2. 25 since RS 3 (and hence RS 2 and RS 1 also) does not hold for $f$. The argument to prove the existence of $P(s)$ with (21) is similar to that for case $L(f)=2$ (cf. p. 163) and we omit the proof.

The situation becomes complicated for case $(3,3)$. Example 2.27 shows that $f \notin \mathfrak{a}: f$, and there exist $C_{l}(s)$ 's in general. The detailed discussion will be found in a subsequent paper of the author.

\section{$\S 14$. Examples of Calculation}

In order to demonstrate how to use the procedures given in the last section, we calculate some examples. The following two examples are $L(f)=2$, and double root occurs.

Example 3. 11. $f=\frac{1}{4}\left(x^{4}+y^{4}+z^{4}\right)-x y \approx \mu=11, \mathfrak{a} \supset \mathfrak{n t}^{5}, \mathfrak{a}: f=\mathfrak{m}$. We use the following notations. $X_{0}=\left(x D_{x}+y D_{y}+z D_{z}\right) / 4, X_{1}=X_{0}$ $+x D_{x} / 4, X_{2}=X_{0}+y D_{y} / 4, X_{3}=X_{0}+\approx D_{z} / 4$ and $\varphi=1-x y \approx$.

$g(s)$ is generated by $X_{i j}$ and following four operators.

$$
\begin{aligned}
& x\left(s-X_{3}\right)-\frac{\approx^{2}}{4 \varphi}\left(y^{2} D_{x}+\approx D_{y}+x^{2} y D_{z}\right), \\
& y\left(s-X_{1}\right)-\frac{x^{2}}{4 \varphi}\left(y^{2} \approx D_{x}+z^{2} D_{y}+x D_{z}\right), \\
& z\left(s-X_{2}\right)-\frac{y^{2}}{4 \varphi}\left(y D_{x}+x z^{2} D_{y}+x^{2} D_{z}\right), \\
& \left(s-X_{1}\right)\left(s-X_{2}\right)-c^{2} x y P_{1} P_{2}-\frac{c x y}{\varphi} \approx\left(3 s-X_{2}+2 X_{0}\right),
\end{aligned}
$$


where

$$
P_{1}=\frac{-1}{\varphi}\left(y^{2} z D_{x}+z^{2} D_{y}+x D_{z}\right)
$$

and

$$
P_{2}=\frac{-1}{\varphi}\left(z^{2} D_{x}+x^{2} z D_{y}+y D_{z}\right)
$$

Then, calculating eigenvectors, we have

$$
\begin{aligned}
& e_{1}=\left(\begin{array}{l}
0 \\
\delta
\end{array}\right), e_{2}=\left(\begin{array}{c}
0 \\
-\delta
\end{array}\right), e_{3}=\left(\begin{array}{c}
D_{x} \delta \\
\frac{-5}{4} D_{x} \delta
\end{array}\right), e_{4}=\left(\begin{array}{c}
D_{y} \delta \\
\frac{-5}{4} D_{y} \delta
\end{array}\right), \\
& e_{5}=\left(\begin{array}{c}
D_{z} \delta \\
\frac{-5}{4} D_{z} \delta
\end{array}\right), e_{6}=\left(\begin{array}{c}
D_{x}^{2} \delta \\
\frac{-3}{2} D_{x}^{2} \delta
\end{array}\right), \cdots, e_{9}=\left(\begin{array}{c}
\left(D_{x}^{3}-6 D_{y} D_{z}\right) \delta \\
\frac{-7}{4}\left(D_{x}^{3}-6 D_{y} D_{z}\right) \delta
\end{array}\right) \cdots
\end{aligned}
$$

By the aid of this basis of $\operatorname{Ham}_{\mathscr{D}}\left(\widetilde{\mathscr{A}}, \mathscr{B}_{p t}\right)$, the action of $s$ can be written

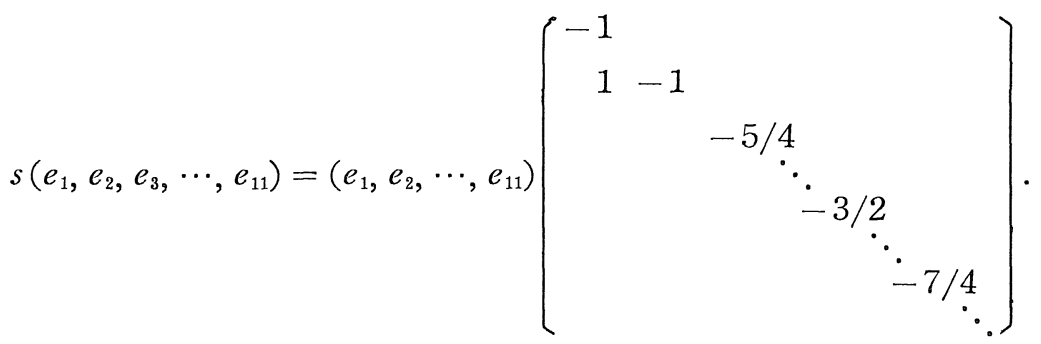

Therefore,

$$
\tilde{b}(s)=(s+1)^{2}\left(s+\frac{5}{4}\right)\left(s+\frac{6}{4}\right)\left(s+\frac{7}{4}\right) .
$$

Example 3. 12. $f=x y\left(x+y^{2}\right)\left(x^{2}+y\right)$

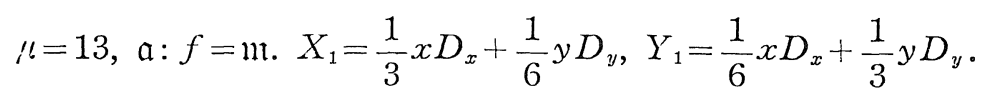

$y(s)$ is generated by three operators:

$$
\begin{gathered}
x\left(s-Y_{1}\right)-\frac{y}{30\left(4-13 x y+9 x^{2} y^{2}\right)}\left\{\left(7 x^{2}-40 y-9 x^{3} y\right) x D_{x}\right. \\
\left.+2\left(14 x^{2}+5 y+18 x^{3} y\right) y D_{y}\right\}
\end{gathered}
$$

and similar operator, exchanging $x$ and $y$ in the above, and 


$$
s^{2}-s A-B, \quad A=\frac{1-\frac{1}{10} x y}{2\left(1-\frac{5}{4} x y\right)}\left(x D_{x}+y D_{y}\right), \quad B=-\frac{1-x y}{1-\frac{5}{4} x y} X_{1} Y_{1}
$$

The operators can be chosen in another way (cf. $\S 19) .\left(\begin{array}{l}0 \\ \delta\end{array}\right)$ and $\left(\begin{array}{c}\delta \\ \frac{-1}{2} \delta\end{array}\right)$ form a root subspace belonging to an eigenvalue -1/2. $\left(\begin{array}{r}D_{x} D_{y} \delta \\ -D_{x} D_{y} \delta\end{array}\right)$ belongs to -1 . Others are $D_{x} \delta, \cdots \leftrightarrow-2 / 3, D_{x}^{2} \delta, \cdots \leftrightarrow-5 / 6, D_{x}^{3} \delta, \cdots$ $\leftrightarrow-1,\left(D_{x}^{2} D_{y}+\frac{1}{6} D_{x}^{4}\right) \delta, \cdots \leftrightarrow-7 / 6,\left(D_{x}^{3} D_{y}-D_{y}^{4}+\frac{1}{20} D_{x}^{5}\right) \delta, \cdots \leftrightarrow-4 / 3$.

Thus,

$$
\tilde{b}(s)=\left(s+\frac{1}{2}\right)^{2}\left(s+\frac{2}{3}\right)\left(s+\frac{5}{6}\right)(s+1)\left(s+\frac{7}{6}\right)\left(s+\frac{4}{3}\right) .
$$

\section{Local Monodromy}

\section{$\S 15 . \quad$ Relation with Local Monodromy}

In the preceding sections, the equality $\operatorname{dim} F=\operatorname{dim} \mathcal{O} / \mathfrak{a}$ holds. This is based on the deep connexion between the local monodromy and the theory of $b$-function.

The local monodromy of $f^{-1}(0)$ around 0 is a linear operator in $H^{i}\left(f^{-1}(\varepsilon) \cap U ; \boldsymbol{C}\right)$, where $U=\left\{x \in \boldsymbol{C}^{n}|| x|<\varepsilon| f,(x) \mid<\delta, 0<\varepsilon \ll \delta<1\right\}$. When $f$ has an isolated singularity at 0 , only $H^{0} \simeq \boldsymbol{C}$ and $H^{n-1} \simeq \boldsymbol{C}^{\mu}$ do not vanish.

In general, the local monodromy and $b$-function relate each other through the hypercohomologies of relative differential form. Since we have not yet completed the argument in general, we discuss here the case of isolated singularity. In this case, the linear map in $H^{n-1}$ is usually called the local monodromy.

Professor B. Malgrange proved in [17],

Theorem 3.13. Let $f$ have an isolated singularity at 0 . Then, $\left.\exp (2 \pi i s)\right|_{F}$ is equivalent to the local monodromy of $f^{-1}(0)$ around 0. 
Therefore, if we determine the action of $s$ in $F$, we can also determine the local monodromy of $f^{-1}(0)$. At this istage, it should be emphasized that, as an invariant of $f^{-1}(0), b$-function is stronger than the Jocal monodromy. For example, let $f_{t}$ be a $\mu$-constant family of isolated singularities. Then, the local monodromy of $f_{0}$ and that of $f_{t}$ are known to be equivalent. $b$-function, however, varies. This situation is extensively analyzed in $\S \S 18,19$. Here we give an easy example.

Example 3. 14. $f=x^{5}+y^{5}+t x^{3} y^{3} . \quad \mu=16$.

For $t=0$, this is weighted homogeneous and

$$
\begin{aligned}
& \tilde{b}(s)=\prod_{\nu=2}^{8}\left(s+\frac{\nu}{5}\right) \\
& \widetilde{B}(s)=\left(s+\frac{2}{5}\right)\left(s+\frac{3}{5}\right)^{2}\left(s+\frac{4}{5}\right)^{3}\left(s+\frac{5}{5}\right)^{4}\left(s+\frac{6}{5}\right)^{3}\left(s+\frac{7}{5}\right)^{2}\left(s+\frac{8}{5}\right) .
\end{aligned}
$$

For $t \neq 0$, the factor $\left(s+\frac{8}{5}\right)$ changes into $\left(s+\frac{3}{5}\right)$ and then

$$
\begin{aligned}
& \widetilde{b}(s)=\prod_{\nu=2}^{7}\left(s+\frac{\nu}{5}\right) \\
& \widetilde{B}(s)=\left(s+\frac{2}{5}\right)\left(s+\frac{3}{5}\right)^{3}\left(s+\frac{4}{5}\right)^{3}\left(s+\frac{5}{5}\right)^{4}\left(s+\frac{6}{5}\right)^{3}\left(s+\frac{7}{5}\right)^{2} .
\end{aligned}
$$

When $t=0, D_{x}^{3} D_{y}^{3} \delta(x, y)$ is an eigenvector belonging to eigenvalue $-8 / 5$, and $\delta(x, y)$ is one belonging to $-2 / 5$. Whereas, when $t \neq 0$, the former cannot be an eigenvector since $f \cdot D_{x}^{3} D_{y}^{3} \delta(x, y) \neq 0$, and the later belongs to two eigenvalues $-2 / 5$ and $-3 / 5$. Local monodromy does not change since $-3 / 5 \equiv-8 / 5 \bmod \boldsymbol{Z}$.

\section{$\S$ 16. Join Formula for $b$-Functions}

Let $f(x)$ and $g(y)$ be holomorphic functions with different variables. Then, we can know the $b$-function of $f(x)+g(y)$ in terms of those of $f$ and $g$. Put $n=\operatorname{dim} X, m=\operatorname{dim} Y$.

Theorem 3. 15. Let $f(x) \in \mathcal{O}_{X}, g(y) \in \mathcal{O}_{Y}, f(0)=g(0)=0$, and assume $g(y)$ is quasi-homogeneous and of isolated singularity. Then, 


$$
\tilde{b}_{f+g}^{n+m}(s) \mid \text { I.c.m. }\left(\tilde{b}_{f}^{n}(s-\alpha) \mid \alpha \in \widetilde{R}_{g}\right) .
$$

Proof. We set $h(x, y)=f(x)+g(y), \mathscr{D}=\mathscr{D}_{X \times Y}, \mathcal{O}=\mathcal{O}_{X \times Y}$ and $Y_{0}$ be the vector field of quasi-homogeneity of $g ; Y_{0} g=g$. Then, the following inclusion holds.

\section{Claim:}

$$
g_{h}(s) \supset g_{f}\left(s-Y_{0}\right) .
$$

The proof of this Claim is given at the last of this section.

Next, since $\mathfrak{a}_{h}+\mathcal{O} h=\mathcal{O} \cdot \mathfrak{a}_{f}+\mathcal{O} f+\mathcal{O} \cdot \mathfrak{a}_{g}$, if we set

$$
\mathscr{H}^{\prime}=\mathscr{D}[s] /\left(\mathscr{D}[s] \mathscr{g}_{f}\left(s-Y_{0}\right)+\mathscr{D}[s] f+\mathscr{D}[s] \mathfrak{a}_{f}+\mathscr{D}[s] \mathfrak{a}_{g}\right) \text {, }
$$

there is a canonical surjection

$$
\mathscr{M}^{\prime} \rightarrow \widetilde{M}_{h} \rightarrow 0 \text {. }
$$

As we know, $\widetilde{\mathscr{H}}_{g}=\mathscr{D}_{Y} / \mathscr{D}_{Y} \mathfrak{a}_{g} \simeq \bigoplus_{i=1}^{\mu} \mathscr{F}_{i}$, where $\mathscr{F}_{i} \simeq \mathscr{B}_{p t}, \quad \mu=\operatorname{dim} \mathcal{O}_{Y} / \mathfrak{a}_{g}$ and $s$ acts on each component separately,

$$
s: u \mapsto \alpha_{i} u, \quad u \in \mathscr{F}_{i},
$$

and $\widetilde{B}_{g}(s)=\Pi\left(s-\alpha_{j}\right)$. Since the action of $s$ is that of $Y_{0}$ in $\widetilde{\mathscr{M}}_{g}$, we have,

$$
\mathscr{M}^{\prime} \simeq \mathscr{D}_{X \times Y} \otimes_{\mathscr{D}_{X}}\left[\bigoplus_{i=1}^{\mu}\left\{\mathscr{D}_{X}[s] /\left(\mathscr{D}_{X}[s] \mathscr{g}_{f}\left(s-\alpha_{i}\right)+\mathscr{D}_{X}[s]\left(\mathfrak{a}_{f}+\mathcal{O}_{X} f\right)\right)\right\}\right]
$$

This proves $\tilde{b}_{h}(s) \mid$ l.c.m. $\left(\tilde{b}_{f}\left(s-\alpha_{j}\right)\right)$.

Corollary 3.16. Upon the conditions of Theorem 3.15, we further assume $f(x)$ is of isolated singularity. Then,

$$
\begin{aligned}
& \tilde{b}_{f+g}(s)=\underset{j}{\operatorname{l.c} . \mathrm{m} .}\left(\tilde{b}_{f}(s-\alpha) \mid \alpha \in \widetilde{R}_{g}\right), \\
& \widetilde{B}_{f+g}(s)=\prod_{i=1}^{\mu} B_{f}\left(s-\alpha_{i}\right) \text {, where } \Pi\left(s-\alpha_{i}\right)=\widetilde{B}_{g}(s) .
\end{aligned}
$$

Proof. In this case, $h=f+g$ has an isolated singularity at $0 \in X \times Y$. $\mathscr{M}^{\prime}$ defined in the proof of Theorem 3.15 satisfies $\overline{S S}\left(\mathcal{M}^{\prime}\right) \subset T_{\{0\}}^{*}(X \times Y)$. We apply the functor $\operatorname{Hom}_{\mathscr{D}}\left(\cdot, \mathscr{B}_{p t \mid X \times Y}\right)$ to (23) and have

$$
0 \rightarrow F_{h} \rightarrow F^{\prime},
$$


where $F_{h}=\operatorname{Ham}_{\mathscr{D}}\left(\widetilde{\mathscr{T}}_{h}, \mathscr{B}_{p t \mid X \times Y}\right), F^{\prime}=\bigoplus_{i=1}^{\prime \prime} \operatorname{Ham}_{\mathscr{D}_{X}}\left(\left.\widetilde{\pi}_{f}\right|_{s \rightarrow s-\alpha_{i}}, \mathscr{B}_{p t}\right)$. Owing to the Theorem 3.13, the equalities

$\operatorname{dim} F_{h}=\operatorname{dim} \Theta / \mathfrak{a}_{h}=\operatorname{dim} \Theta_{X} / \mathfrak{a}_{f} \times \operatorname{dim} \Theta_{Y} / \mathfrak{a}_{g}=\operatorname{dim} F^{\prime}$

holds. Therefore, (24) is an isomorphism.

Q.E.D.

Corollary 3. 17. When $f(x)$ has an isolated singularity at $0 \in X$, the b-function of $h(x, y)=f(x)+\sum_{i=1}^{k} y_{i}^{2}$ at $0 \in X \times Y$, is

$$
\tilde{b}_{h}(s)=\tilde{b}_{f}\left(s+\frac{k}{2}\right), \quad \widetilde{B}_{h}(s)=\widetilde{B}_{f}\left(s+\frac{k}{2}\right) .
$$

We note that there is an isomorphism, in Corollary 3.16,

$$
\mathscr{M}^{\prime} \underset{\rightarrow}{\rightarrow} \widetilde{M}_{h}
$$

by applying the functor $\operatorname{Ham}_{\boldsymbol{C}}\left(\cdot, \mathscr{B}_{p t \mid X \times Y}\right)$ to (24). This isomorphism can be proved (and hence also $3 \cdot 16$ ) directly, i.e. without using Theorem 3. 13 , when $L(f) \leq 2$, or, case $(2,3$; a).

In order to prove Claim in the proof of Theorem 3.15, we prepare

Lemma 3. 18. There are natural numbers $c_{j}^{(l)} j \geq 0$ such that

$$
(s)_{h}=\sum_{j=0}^{h}(-)^{j} c_{j}^{(l)}(h)_{j}(s+l)_{h-j}, \quad 1 \leq l .
$$

Proof. We use the induction on $l$. When $l=1$, one can prove by induction on $h$ that

$$
(s)_{h}=\sum_{j=1}^{h}(-)^{j}(h)_{j}(s+1)_{h-j} .
$$

Then, it follows from the hypothesis of induction and (25) that

$$
\begin{aligned}
(s)_{h} & =\sum_{j}(-)^{j} c_{j}^{(l)}(h)_{j}(s+l)_{h-j} \\
& =\sum_{j}(-)^{j} c_{j}^{(l)}(h)_{j} \sum_{k=0}^{h-j}(-)^{k}(h-j)_{k}(s+l+1)_{h-j-k} \\
& =\sum_{j}(-)^{j+k} c_{j}^{(l)}(h)_{j+k}(s+l+1)_{h-j-k} . \quad \text { Q.E.D. }
\end{aligned}
$$

$c_{j}^{(l)}$ are determined by 


$$
\begin{aligned}
& c_{j}^{(0)}=0, \quad j \neq 0, \quad c_{0}^{(l)}=1, \quad l=0,1, \cdots \\
& c_{j+1}^{(l)}=c_{j}^{(l)}+c_{j+1}^{(l-1)} .
\end{aligned}
$$

Choose an operator of the form

$$
P(s, x, D)=\sum_{k=0}^{m}(s-k)_{m-k} p_{k}(x, D) \quad \text { with } \quad p_{k}(x, D)=\sum_{|\alpha|=k} a_{k, \alpha} D^{\alpha} .
$$

We apply this Lemma 3.18 for $\left(s-Y_{0}-k\right)_{m-k}$ in the following formula.

$$
\begin{aligned}
& P\left(s-Y_{0}, x, D\right)(f+g)^{s} \\
& =\sum_{l \sum^{k} \geq 0}(s)_{k-l}\left(s-Y_{0}-k\right)_{m-k} R_{l}\left[p_{k}\right](x, d f)(f+g)^{s-k+l} \\
& =\sum(s)_{k-l}\left(\sum_{j}(-)^{j} c_{j}^{(l)}(m-k)_{j}\left(s-Y_{0}-k+l\right)_{m-k-j}\right) \\
& \quad \times R_{l}\left[p_{k}\right](f+g)^{s-k+l} \\
& =\sum(s)_{k-l} \sum(-)^{j} c_{j}^{(l)}(m-k)_{j}(s-k+l)_{m-k-j} f^{m-k-j} \\
& \quad \times R_{l}\left[p_{k}\right](f+g)^{s-m+l+j} \\
& =\sum(s)_{m-l-j}(-)^{j} c_{j}^{(l)} \sum_{k}(m-k)_{j} f^{m-k-j} R_{l}\left[p_{k}\right](f+g)^{s-m+l+j} \\
& =\sum(s)_{m-l-j}(-)^{j} c_{j}^{(l)} R_{l}\left[\frac{\partial^{j}}{\partial s^{j}} \sigma^{T}(p)\right](f, x, d f)(f+g)^{s-m+l+j} .
\end{aligned}
$$

Similarly,

(27) $P(s, x, D) f^{s}$

$$
=\sum(s)_{m-l-j}(-)^{j} c_{j}^{(l)} R_{l}\left[\frac{\partial^{j}}{\partial s^{j}} \sigma^{T}(p)\right](f, x, d f) f^{s-m+l+j} .
$$

Any $P(s) \in \mathscr{D}_{X}[s]$ can be uniquely written as

$$
P(s, x, D)=\sum_{\mu=0}^{m} P_{\mu}(s, x, D),
$$

where

$$
\begin{aligned}
& P_{\mu}(s, x, D)=\sum_{k=0}^{\mu}(s-j)_{\mu-k} p_{\mu, k}(x, D), \\
& p_{\mu, k}(x, D)=\sum_{|\alpha|=k} a_{\mu, k, \alpha} D^{\alpha} .
\end{aligned}
$$

Then, the preceding equalities (26) and (27) say that the coefficient of $(s)_{d}(f+g)^{s-d}$ in $P\left(s-Y_{0}, x, D\right)(f+g)^{s}$ and that of $(s)_{d} f^{s-d}$ in 
$P(s, x, D) f^{s}$ are the same. Therefore, $P\left(s-Y_{0}, x, D\right) \in g_{f+g}(s)$ when $P(s, x, D) \in g_{f}(s)$. Q.E.D. of Claim.

\section{Chapter IV. Results of $b(s)$}

In this chapter, we investigate several examples of $b$-functions. Sections $18 \sim 20$ are devoted to the study of non-quasi-homogeneous isolated singularities in $\operatorname{dim} X=2,3$. We add some remarks in $\S 21$, about the $b$-functions of isolated singularities with modality not greater than 2. Its detailed arguments in case corank $(f)=3$ will be found in [32]. Examples of non-isolated singularities are given in $\S 22$.

\section{$\S 17$. Two-Dimensional Case}

When the space dimension is 2 , we can apply Theorem 2.24. As is shown below, we find "explicit formulae" under some assumptions on $f$. Let us explain the situation.

First, we assume that $f$ is a locally reduced non-quasi-homogeneous function at $0 \in C^{2}$ such that

(a) $\mathfrak{a}: f=\left(x^{a}, y^{b}\right)$.

Next, we assume that generators of $g(s) \cap(\mathscr{D} s+\mathscr{D})$ are given by

$$
A_{1}(s, x, D)=x^{a}\left(s-X_{2}\right)+A_{1}^{\prime}(x, D),
$$

and

$$
A_{2}(s, x, D)=y^{b}\left(s-X_{1}\right)+A_{2}^{\prime}(x, D),
$$

where $X_{k}=a_{k 1} x D_{x}+a_{k 2} y D_{y}, \quad k=1,2, \quad a_{i j} \in \mathbb{Q}^{+}$, and they satisfy the condition

(b) the weight of $A_{1}^{\prime}(x, D) \quad\left(A_{2}^{\prime}(x, D)\right.$, respectively) is greater than that of $a_{21} a$ in $X_{2}$ ( $a_{12} b$ in $X_{1}$, respectively).

Set $A=\left[\begin{array}{ll}a_{11} & a_{12} \\ a_{21} & a_{22}\end{array}\right]$. There are two cases.

$1^{\circ} \operatorname{rank} A=1$.

Assume $\left(a_{11}, a_{12}\right)=c \cdot\left(a_{21}, a_{22}\right) \quad c \in Q$. We write $f$ in the following form: $f=f_{0}+g$, where $f_{0}$ is the sum of monomials in $f$ which have 
minimal weight, say $r c^{\prime}$, with respect to $\left(a_{21}, a_{22}\right)$. Then,

$$
\begin{aligned}
& x^{a}\left(f_{0}+g\right)=x^{a}\left(w f_{0}+X_{2} g\right)-A_{1}^{\prime}(x, D)\left(f_{0}+g\right) \\
& y^{b}\left(f_{0}+g\right)=y^{b}\left(c w f_{0}+c X_{2} g\right)-A_{2}^{\prime}(x, D)\left(f_{0}+g\right) .
\end{aligned}
$$

Comparing the terms with minimal weight in these formulae, we have $r=1$ and $c=1$. Thus, $X_{1}=X_{2}=X_{0}=\alpha x D_{x}+\beta y D_{y}, f=f_{0}+g$, $X_{0} f_{0}=f_{0}$ and $g$ has the weight greater than 1 with respect to $X_{0}$. This shows that, when $\operatorname{rank} A=1, f$ can be considered as a higher order deformation of weighted homogeneous polynomial. Since $y^{b} A_{1}-x^{a} A_{2}$ $=\varphi\left(f_{x} D_{y}-f_{y} D_{x}\right), \varphi(0) \neq 0$, we have

$$
1>(a+1) \alpha+(b+1) \beta .
$$

$2^{\circ} \operatorname{rank} A=2$.

In this case, inequalities $a_{11} \neq a_{21}, a_{12} \neq a_{22}$ holds in general. Then the relation $x^{a} A_{2}-y^{b} A_{1}=\varphi\left(f_{x} D_{y}-f_{y} D_{x}\right)$ again shows

$$
\begin{aligned}
1 & =(a+1) a_{11}+(b+1) a_{12} \\
& =(a+1) a_{21}+(b+1) a_{22} .
\end{aligned}
$$

That is, $A$ can be written in the form

$$
A=\left(1-(a+1) a_{21}-(b+1) a_{12}\right)\left[\begin{array}{ll}
\frac{1}{a+1} & \\
& \frac{1}{b+1}
\end{array}\right]+\left[\begin{array}{l}
1 \\
1
\end{array}\right]\left(a_{21}, a_{12}\right) .
$$

Taking the determinant of coefficients of $\left[\begin{array}{c}A_{1} \\ A_{2}\end{array}\right]$, we have

$$
f=c \cdot x^{a+1} y^{b+1}+g, \quad c \neq 0,
$$

where $g$ is the sum of monomials whose weight are strictly greater than that of $x^{a+1} y^{b+1}$ with weight of $X_{1}$ or $X_{2}$.

Moreover, we impose the condition

(c) $L(f)=2$.

Upon these conditions, we conjecture that the action of $s$ is determined by $a, b$ and $A$. The explicit formulae for $b$-functions are given as follows. 


\section{Conjecture 4.0 (EEF).}

$1^{\circ} \operatorname{rank} A=1 . \quad A=\left[\begin{array}{l}1 \\ 1\end{array}\right](\alpha, \beta)$. Then, $\prime=\frac{1-\alpha}{\alpha} \cdot \frac{1-\beta}{\beta}$,

$$
\widetilde{P}(t)=\frac{\left(t^{\alpha}-t\right)\left(t^{\beta}-t\right)}{\left(1-t^{\alpha}\right)\left(1-t^{\beta}\right)}+t^{1-a \alpha-b \beta}(1-t) \frac{\left(1-t^{a \alpha}\right)\left(1-t^{b \beta}\right)}{\left(1-t^{\alpha}\right)\left(1-t^{\beta}\right)} \text {. }
$$

Moreover, $s$ is semisimple.

$2^{\circ} \operatorname{rank} A=2$.

$$
A=\left[\begin{array}{ll}
\beta^{\prime} & \beta \\
\alpha & \alpha^{\prime}
\end{array}\right]=(1-(a+1) \alpha-(b+1) \beta)\left[\begin{array}{ll}
\frac{1}{a+1} & \\
& \frac{1}{b+1}
\end{array}\right]+\left[\begin{array}{l}
1 \\
1
\end{array}\right](\alpha, \beta) .
$$

Then, $\iota=1+\frac{b}{\alpha}+\frac{a}{\beta}$, and

$$
\widetilde{P}(t)=t+\frac{t^{\alpha+\alpha^{\prime}}}{(1-t)(1-t)\left(1-t^{b \alpha^{\prime}}\right)}+\frac{t^{\beta+\beta^{\prime}}(1-t)\left(1-t^{\alpha \beta^{\prime}}\right)}{\left(1-t^{\beta}\right)\left(1-t^{\beta^{\prime}}\right)} .
$$

Set $d=$ g.c.d. $(a+1, b+1)$. Then, $-\frac{\nu}{d}, \nu=1, \cdots, d-1$ are non semisimple eigenvalue of $s$ of height two.

We call the formula and proviso about semisimplicity of $s$ in $1^{\circ}$ (respectively in $2^{\circ}$ ) as "EEF" type $1^{\circ}$ (resp. $2^{\circ}$ ).

The common case where formulae type $1^{\circ}$ and $2^{\circ}$ could cover formally is the following.

$$
A=\left[\begin{array}{l}
1 \\
1
\end{array}\right](\alpha, \beta) \text { and } 1=(a+1) \alpha+(b+1) \beta .
$$

In this case, even though these two formulae seem very different, they give the same result as directly seen. Of course, this case can never occur according to the restriction (2).

We also conjecture that a second order operator showing that $L(f)=2$ can be chosen in the following way

Type $1^{\circ}$

$$
\begin{aligned}
& \left(s-X_{0}+c^{\prime}\right)\left(s-X_{0}\right)+s A^{\prime}+B^{\prime}, \\
& c^{\prime}=1-(a+1) \alpha-(b+1) \beta,
\end{aligned}
$$


where each term in $s A^{\prime}+B^{\prime}$ has strictly positive weight with respect to $X_{0}$. Note that $c^{\prime}$ is positive by the inequality (2).

Type $2^{\circ}$

$$
\left(s-X_{1}\right)\left(s-X_{2}\right)+s A^{\prime}+B^{\prime},
$$

where $s A^{\prime}+B^{\prime}$ has strictly positive weight with respect to both $X_{1}$ and $X_{2}$.

Especially when $a=b=1$ in type $1^{\circ}$, we can also take

$$
(s+1-\alpha-\beta)\left(s-X_{0}\right)+s A^{\prime}+B^{\prime}+\left(\alpha D_{x} A_{1}^{\prime}+\beta D_{y} A_{2}^{\prime}\right) .
$$

We add some remarks to "EEF" type $1^{\circ}$.

According to the analysis in case $1^{\circ}$,

$$
f=f_{0}+(\text { higher weight }), X_{0} f_{0}=f_{0} \text {. }
$$

The first term of $\widetilde{P}_{f}(t)$ of type $1^{\circ}$ is the same with $\widetilde{P}_{f_{0}}(t)$. Since there is a factor $(1-t)$ in the second term, $\widetilde{P}_{f}(t)$ and $\widetilde{P}_{f_{0}}(t)$ can be expanded into the fractional polynomial of the form:

$$
\widetilde{P}_{f}(t)=\sum_{r \in C_{0}} t^{r}+\sum_{r \in C} t^{r},
$$

and

$$
\widetilde{P}_{f_{0}}(t)=\sum_{r \in C_{0}} t^{r}+\sum_{r \in C} t^{r+1}
$$

Note that

$$
\min C_{0}<\min C
$$

owing to the inequality (2), because $\min C_{0}=\alpha+\beta$ and $\min C=1-a \alpha$ $-b \beta$.

There is a natural generalization of "EEF" type $1^{\circ}$ to $n$-dimensional case. That is, if
(a) ${ }_{n}$
$\mathfrak{a}: f=\left(x_{1}^{a_{1}}, \cdots, x_{n}^{a_{n}}\right)$,

and first order operators associated to it are

$$
x_{i}^{a_{i}}\left(s-X_{0}\right)+(\text { higher weight }),
$$

with $X_{0}=\sum \alpha_{i} x_{i} D_{i}$, and
(c) ${ }_{n}$
$L(f)=2$, 
then

$$
\widetilde{P}(t)=\Pi \frac{t^{\alpha_{i}}-t}{1-t^{\alpha_{i}}}+t^{(n-1)-\Sigma a_{i} \alpha_{i}}(1-t) \Pi \frac{1-t^{a_{i} \alpha_{i}}}{1-t^{\alpha_{i}}} .
$$

Moreover, $s$ is semisimple.

We refer this formula with proviso as "EEF" type $1_{(n)}$. There are several cases where these conjectures can be verified, as we will discuss later on.

Conditions (a), (b) and (c) are essential. In fact, types $W_{1,2 q}^{\#}$ and $W_{1,2 q-1}^{\#}$ in $\S 21$ satisfy (a) (with $a=1, b=2$ ) and (c) but violates (b). $\widetilde{P}(t)$ is given in [32] and is different from both type $1^{\circ}$ and $2^{\circ}$.

The next example satisfies (a) and (b) but violates (c).

Example 4. 1. $f=\frac{1}{n_{1}} x^{n_{1}}+\frac{1}{n_{2}}\left(y-t x^{m_{1}}\right)\left(y+\left(n_{2}-1\right) t x^{n_{1}}\right)^{n_{2}-1}$.

$t$ is a non-zero parameter. We impose conditions

$$
\left(n_{1}-1\right) / 4 \leq m_{1} \leq\left(n_{1}-2\right) / 3,1 / n_{2}<m_{1} / n_{1} .
$$

Then $\mathfrak{a}: f=\left(x^{n_{1}-2 m_{1}-1}, y\right)$, and first order operators associated with it are the following: $\left(m_{2}=n_{2}-1\right)$

$$
\begin{aligned}
& y\left(s-X_{0}+\left(m_{1} m_{2}-c\right) t x^{m_{1}} D_{y}\right)-c m_{2} t^{2} x^{2 m_{1}} D_{y}, \\
& x^{n_{1}-2 m_{1}-1}\left(s-X_{0}+\left(m_{1} m_{2}-c\right) t x^{m_{1}} D_{y}\right)-c m_{2} t^{2} \frac{\left(y+m_{2} t x^{m_{1}}\right)^{n_{2}-5}}{\varphi} Q,
\end{aligned}
$$

where

$$
\begin{aligned}
Q= & \left(y+m_{2} t x^{m_{1}}\right)^{3}\left(D_{x}-m_{1} m_{2} t x^{m_{1}-1} D_{y}\right) \\
& +\frac{m_{1} t x^{m_{1}-1}}{y}\left\{\left(y^{4}+m_{2} t x^{m_{1}}\right)^{4}-\left(m_{2} t x^{m_{1}}\right)^{4}\right\} D_{y} \\
\varphi= & 1-m_{1} m_{2}^{4} t^{5} x^{5 m_{1}-n_{1}}\left(y+m_{2} t x^{m_{1}}\right)^{n_{2}-5} .
\end{aligned}
$$

Owing to the inequality $1 / n_{2}<m_{1} / n_{1}$, we can check the condition (b). However, condition (c) does not hold. In fact, $2=l(f)<L(f)=3$ in this case. $\widetilde{P}(t)$ is given by the following and does not coinside with formula type $1^{\circ}$ or $2^{\circ}$.

$$
\widetilde{P}(t)=\frac{\left(t^{1 / n_{1}}-t\right)\left(t^{1 / n_{2}}-t\right)}{\left(1-t^{1 / n_{1}}\right)\left(1-t^{1 / n_{2}}\right)}+t^{1-\left(n_{1}-2 m_{1}-1\right) / n_{1}-1 / n_{2}}(1-t) \frac{1-t^{\left(n_{1}-2 m_{1}\right) / n_{1}}}{1-t^{1 / n_{1}}}
$$




$$
+t^{1-\left(n_{1}-3 m_{1}-1\right) / n_{1}-2 / n_{2}}(1-t) \frac{1-t^{\left(n_{1}-3 m_{1}\right) / n_{1}}}{1-t^{1 / n_{1}}} .
$$

See type $X_{0}^{b p}$ in $\S 18$ and [32].

$$
\S \text { 18. } x^{n_{1}}+y^{n_{2}}+x^{m_{1}} y^{m_{2}}
$$

In this section, we study the typical example

$$
f(x, y)=\frac{1}{n_{1}} x^{n_{1}}+\frac{1}{n_{2}} y^{n_{2}}-t x^{m_{1}} y^{m_{2}},
$$

where $t$ is a parameter.

We can assume $1 \leq m_{i} \leq n_{i}-1$ owing to Proposition 2.10. In the following, $c$ always denotes $\sum \frac{m_{i}}{n_{i}}-1$.

When $c=0, f$ is weighted homogeneous polynomial with weight $\left(\frac{1}{n_{1}}, \frac{1}{n_{2}}\right)$, and hence by Theorem 3.6 , we have

$$
\widetilde{P}(t)=\frac{\left(t^{1 / n_{1}}-t\right)\left(t^{1 / n_{2}}-t\right)}{\left(1-t^{1 / n_{1}}\right)\left(1-t^{1 / n_{2}}\right)}
$$

When $c \neq 0, f$ is of simplex type, and when $c>0, f$ is a $\mu$-constant deformation of

$$
\frac{1}{n_{1}} x^{n_{1}}+\frac{1}{n_{2}} y^{n_{2}}
$$

Therefore, the local monodromy of $f$ is the same as that of (8). But $\widetilde{P}(t)$ is not given by $(7)$ as is shown below. When $c<0, b_{f}(s)$ may have double roots. Then so do the local monodromy. In the sequel, we use the following notation.

$$
\begin{aligned}
& X_{0}=\frac{1}{n_{1}} x D_{x}+\frac{1}{n_{2}} y D_{y}, X_{1}=\frac{\left(n_{2}-m_{2}\right)}{m_{1} n_{2}} x D_{x}+\frac{1}{n_{2}} y D_{y}, \\
& X_{2}=\frac{1}{n_{1}} x D_{x}+\frac{\left(n_{1}-m_{1}\right)}{m_{2} n_{1}} y D_{y} .
\end{aligned}
$$

First of all, we determine $g(s) \cap(\mathscr{D} s+\mathscr{D})$.

Proposition 4.2. $\mathfrak{a}: f$ are $g(s) \cap(\mathscr{D} s+\mathscr{D})$ are given as follows. 1. $1 \leq m_{1} \leq n_{1} / 2,1 \leq m_{2} \leq n_{2} / 2:\left(x^{m_{1}-1}, y^{m_{2}-1}\right)$ 


$$
\begin{aligned}
& x^{m_{1}-1}\left(s-X_{2}\right)+\frac{c}{m_{1} m m_{2} t} y^{n_{2}-m_{2}} D_{x}-\frac{1}{m_{1} m_{2} t^{2}} x^{n_{1}-m_{1}-1} y^{n_{2}-2 m_{2}}\left(s-X_{0}\right), \\
& y^{m_{2}-1}\left(s-X_{1}\right)+\frac{c}{m_{1} m_{2} t} x^{n_{1}-m_{1}} D_{y}-\frac{1}{m_{1} m_{2} t^{2}} x^{n_{1}-2 m_{1}} y^{n_{2}-m_{2}-1}\left(s-X_{0}\right) .
\end{aligned}
$$

2. $n_{1} / 2 \leq m_{1} \leq n_{1}-1, n_{2} / 2 \leq m_{2} \leq n_{2}-1:\left(x^{n_{1}-m_{1}-1}, y^{n_{2}-m_{2}-1}\right)$

$$
\begin{aligned}
& x^{n_{1}-m_{1}-1}\left(s-X_{0}\right)-c t y^{m_{2}} D_{x}-m_{1} m m_{2} t^{2} x^{m_{1}-1} y^{2 m_{2}-n_{2}}\left(s-X_{2}\right), \\
& y^{n_{2}-m_{2}-1}\left(s-X_{0}\right)-c t x^{m_{1}} D_{y}-m_{1} m m_{2} t^{2} x^{2 m_{1}-n_{1}} y^{m_{2}-1}\left(s-X_{1}\right) .
\end{aligned}
$$

3. $n_{1}+1 \leq 2 m_{1}, n_{2}-1 \geq 2 m_{2}$ :

$$
\begin{aligned}
& \left(\left(x^{n_{1}-m_{1}}-m_{1} t y^{m_{2}}\right),\left(x^{n_{1}-m_{1}-1} y^{n_{2}-2 m_{2}-1}-m_{1}^{2} m_{2} t^{3} x^{2 m_{1}-n_{1}-1} y^{m_{2}-1}\right)\right) . \\
& x^{n_{1}-m_{1}}\left(s-X_{0}\right)-m_{1} t y^{m_{2}}\left(s-X_{1}\right), \\
& y^{n_{2}-2 m_{2}-1}\left\{x^{n_{1}-m_{1}-1}\left(s-X_{0}\right)-c t y^{m_{2}} D_{x}\right\} \\
& \quad \quad-m_{1}^{2} m_{2} t^{3} x^{2 m_{1}-n_{1}-1}\left\{y^{m_{2}-1}\left(s-X_{1}\right)+\frac{c}{m_{1} m_{2} t} x^{n_{1}-m_{1}} D_{y}\right\} .
\end{aligned}
$$

4. $n_{2}+1 \leq 2 m_{2}, n_{1}-1 \geq 2 m_{1}$ : $\mathfrak{a}: f$ and first order operators are the same with those of case 3 , if we exchange $x$ and $y$, and subscripts 1 and 2.

Proof. One can prove by direct calculation that the operators listed above belong to $\mathscr{f}(s)$. The proof that they actually form a basis is based on Theorem 1. 10. We rewrite them in the form $a_{\nu}(x) s+a_{\nu 1}(x) D_{x}$ $+a_{\nu_{2}}(x) D_{y}$, and calculate $d=\operatorname{det}\left[\begin{array}{ll}a_{11} & a_{12} \\ a_{21} & a_{22}\end{array}\right]$. Then, we have

$$
d=\left\{\begin{array}{ll}
c t\left(1-m_{1} m_{2} t^{2} x^{2 m_{1}-n_{1}} y^{2 m_{2}-n_{2}}\right) f & \cdots 2 \\
-\frac{c}{m_{1} m_{2} t}\left(1-\frac{1}{m_{1} m_{2} t^{2}} x^{n_{1}-2 m_{1}} y^{n_{2}-2 m_{2}}\right) f & \cdots 1 \\
m_{1} c t^{2} f & \cdots 3 \\
m_{2} c t^{2} f & \cdots 4
\end{array} .\right.
$$

Thus they form a basis.

Q.E.D.

In general, $L(f) \leq \min \left(n_{1}, n_{2}\right) \quad$ (cf. Example 3.18).

There are special types of functions for which $L(f) \leq 2$ holds. They are listed in the following table. We denote $a \rightarrow b$ under $m_{\nu}$, to indicate $a \leq m_{\nu} \leq b$. 
Table 4.3

i) $c<0$
1. $X_{1}$
2. $X_{1}^{\#}$
3. $X_{2}$
4. $X_{2}^{\#}$
5. $S$
6. $S$ \#

ii) $c>0$

7. $X_{0}$

8. $X_{0}^{b}$

9. $Y$

10. $Y^{b}$

\section{$m_{1}$}

$1 \rightarrow\left(n_{1}+1\right) / 2$

$\left(n_{1}+2\right) / 2 \rightarrow\left(2 n_{1}+1\right) / 3$

1

1

$2 \rightarrow n_{1} / 2$

$2 \rightarrow n_{1} / 3$

and

$\left(n_{1}+1\right) / 2$

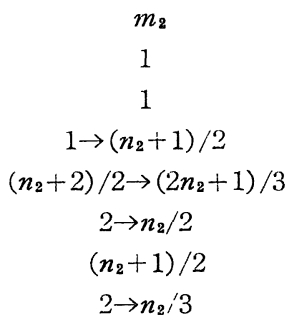

$$
\begin{gathered}
n_{2}-1 \\
\left(n_{2}-1\right) / 2 \rightarrow n_{2}-1 \\
n_{2}-1 \\
\left(n_{2}-1\right) / 3 \rightarrow\left(n_{2}-2\right) / 2 \\
n_{2} / 2 \rightarrow n_{2}-2 \\
\left(n_{2}-1\right) / 2 \\
(2 / 3) n_{2} \rightarrow n_{2}-2
\end{gathered}
$$

iii) Special cases of i) and ii)
11. $X_{1, \min }^{\#}$
$\left(n_{1}+2\right) / 2$
1
12. $X_{2}^{\#}$, min
1
$\left(n_{2}+2\right) / 2$
13. $S_{\min }\left(S_{\text {min }}^{\text {\#in }}\right)$
2
14. $X_{0, \max }^{b}$
$\left(n_{1}-2\right) / 2$
2
$n_{2}-1$
$\left(n_{2}-1\right) / 2$
15. $Y_{\max }\left(Y_{\max }^{b}\right)$

$$
\text { and } \quad n_{1}-1
$$$$
n_{2}-2
$$
$S_{\min }^{\#}$ and $Y_{\max }^{b}$ appear only when $\min \left(n_{1}, n_{2}\right)=3$.

Using the notations above we can state the following

Theorem 4.4. 1) The function $f$ enjoys the property $1 A(2 A$, $3 A$, respectively) if and only if it has the property $1 B(2 B, 3 B$, respectively).

A B

1 Quasi-homogeneous

$X_{0}, X_{1}, X_{2}$, or $c=0$.

$2 \quad \mathfrak{a}: f=\left(x^{a}, y^{b}\right) \quad a \geq 1, b \geq 1 . \quad X_{1}^{\#}, X_{2}^{\#}, S, S^{\#}, X_{0}^{b}, Y, Y^{b}$.

$3 a: f=(x, y)$ $X_{1, \min }^{\#}, X_{2, \min }^{\#}, S_{\min }, S_{\min }^{\#}$ $X_{0, \max }^{b}, Y_{\max }, Y_{\max }^{b}$.

2) When $f$ has the property that $\mathfrak{a}: f=\left(x^{a}, y^{b}\right), a \geq 1, b \geq 1$, in 1) the equality $L(f)=2$ holds.

Proof. 1): 1A. If $f$ is of type $X_{0}$ and $m_{1}=n_{1}-1, m_{2} \geq n_{2} / 2$, then use 2 of Proposition 5. 2. If $m_{1}=n_{1}-1, m_{2}=\left(n_{2}-1\right) / 2$, then use 3 . If $f$ 
is of type $X_{1}$ and $m_{2}=1, m_{1} \leq n_{1} / 2$, then use 1 . If $m_{2}=1, m_{1}=\left(n_{1}+1\right) / 2$, then use 3. The proof for the case $X_{2}$ can be done in the same manner. $2 \mathrm{~A}$ and $3 \mathrm{~A}$. These can be derived from Proposition 5.2 by direct calculation.

To prove 2), we list up second order operators, which certificate that $L(f)=2$.

$S: \quad\left(s-X_{1}\right)\left(s-X_{2}\right)-\frac{1}{m_{1} m_{2} t^{2}} x^{n_{1}-2 m_{1}} y^{n_{2}-2 m_{2}}\left(s-X_{0}+c\right)\left(s-X_{0}\right)$.

$Y: \quad\left(s-X_{0}+c\right)\left(s-X_{0}\right)-m_{1} m_{2} t^{2} x^{2 m_{1}-n_{1}} y^{2 m_{2}-n_{2}}\left(s-X_{1}\right)\left(s-X_{2}\right)$.

$S^{\#}:\left(s-X_{1}\right)\left(s-X_{2}\right)-\frac{1}{m_{1} m_{2}^{2} t^{3}} x^{n_{1}-3 m_{1}}\left(s-X_{0}+c+\frac{m_{1}}{n_{1}}-\frac{1}{n_{2}}\right)$

$$
\times\left\{y^{n_{2}-m_{2}-1}\left(s-X_{0}\right)-c t x^{m_{1}} D_{y}\right\} \quad\left(m_{2}=\left(n_{2}+1\right) / 2\right) .
$$

$X_{1}^{\#}:\left(s-X_{1}-\frac{n_{1}}{m_{1}} c\right)\left\{\left(s-X_{1}\right)+\frac{c}{t m_{1}} x^{n_{1}-m_{1}} D_{y}\right\}-Q(s)$,

$$
Q(s)=\left\{\begin{array}{l}
\frac{1}{m_{1}^{2} t^{3}} x^{2 n_{1}-3 m_{1}} y^{n_{2}-3}\left(s-x_{0}+c\right)\left(s-X_{0}\right), \\
\frac{1}{m_{1}^{3} t^{4}} x^{2 n_{1}-3 m_{1}+1} y^{n_{2}-4}\left(s-X_{0}+c-\frac{1}{n_{1}}+\frac{1}{n_{2}}\right) \\
\quad \times\left\{x^{n_{1}-m_{1}-1}\left(s-X_{0}\right)-\operatorname{cty} D_{x}\right\} .
\end{array}\right.
$$

$Y^{\natural}:\left(s-X_{0}+c\right)\left(s-X_{0}\right)-m_{1}^{2} m_{2} t^{3} x^{3 m_{1}-2 n_{1}}$

$$
\begin{gathered}
\times\left(s-X_{1}-\frac{n_{1}}{m_{1}} c+\frac{m_{2}}{n_{2}}-\frac{1}{n_{2}}\right)\left\{y^{m_{2}-1}\left(s-X_{1}\right)+\frac{c}{m_{1} m_{2} t} x^{n_{1}-m_{1}} D_{y}\right\} \\
\left(m_{2}=\left(n_{2}-1\right) / 2\right) .
\end{gathered}
$$

$X_{0}^{\natural}:\left(s-X_{0}+c+\frac{m_{1}}{n_{1}}-\frac{1}{n_{2}}\right)\left(s-X_{0}-c t x \frac{m_{1}}{n_{1}} D_{y}\right)-Q(s)$,

$$
Q(s)=\left\{\begin{array}{l}
m_{1} m_{2}^{2} t^{3} x^{3 m_{1}-n_{1}} y^{n_{2}-3}\left(s-X_{1}\right)\left(s-X_{2}\right) \\
m_{2}^{3} t^{3} x^{3 m_{1}-n_{1}+1} y^{n_{2}-4}\left(s-X_{2}-\frac{c}{m_{2}}-\frac{1}{n_{1}}+\frac{1}{n_{2}}\right) \\
\quad \times\left\{m_{1} t x^{m_{1}-1}\left(s-X_{2}\right)+\frac{c}{m_{2}} y D_{x}\right\} \quad\left(m_{2}=n_{2}-1\right) .
\end{array}\right.
$$

The operator for $X_{2}^{\#}$ is similar to that of $X_{1}^{\#}$. In view of these operators, we can conclude that $L(f)=2$. Q.D. 
Note that second order operators in case 1), 3 can be taken in the form (cf. 175)

$$
(s+1-\alpha-\beta)\left(s-X_{0}\right)+\cdots .
$$

For instance, in type $Y_{\max }, m_{1}=n_{1}-2, m_{2}=n_{2}-2$,

$$
\begin{aligned}
(s+ & \left.1-\frac{1}{n_{1}}-\frac{1}{n_{2}}\right)\left(s-X_{0}\right)+\frac{m_{1} m_{2} t^{2}}{c} x^{n_{1}-4} y^{n_{2}-4}\left(s-X_{1}\right)\left(s-X_{2}\right) \\
& +\frac{c t^{2}}{\varphi} x^{n_{1}-5} y^{n_{2}-5}\left\{\frac{m_{1}}{n_{1}} x^{2} P_{1}+\frac{m_{2}}{n_{2}} y^{2} P_{2}+\left(\frac{m_{1}}{n_{1}}+\frac{m_{2}}{n_{2}}\right) \frac{m_{1} m_{2}}{c} x y\left(s-X_{0}\right)\right\} \\
& -c t\left\{\frac{y^{n_{2}-4}}{n_{1}} P_{1}^{2}+\frac{x^{n_{1}-4}}{n_{2}} P_{2}^{2}\right\}, \\
P_{1}= & \frac{-1}{\varphi}\left(y D_{x}+m_{1} t x^{n_{1}-3} D_{y}\right), \quad P_{2}=\frac{-1}{\varphi}\left(m_{2} t y^{n_{2}-3} D_{x}+x D_{y}\right), \\
\varphi= & 1-m_{1} m_{2} t^{2} x^{n_{1}-4} y^{n_{2}-4} .
\end{aligned}
$$

We exibit some special cases.

1. $n_{1}=3$. Then $f$ is quasi-homogeneous or $L(f)=2$ : types $X_{2}^{\#}, Y^{b}$, $X_{0}^{b}, S^{\#}$.

2. $n_{1}=4$. Then $f$ is quasi-homogeneous or $L(f)=2$, except next three cases.

(1) $m_{1}=3 ;\left(n_{2}-1\right) / 3>m_{2} \geq 2, m_{2} \neq n_{2} / 4$.

They are case $(2,3 ; a)$.

\begin{tabular}{|c|c|c|c|c|}
\hline & $m_{1}$ & $m_{2}$ & condition & $a^{\prime}$ \\
\hline$S \# \#$ & $2 \rightarrow n_{1} / 4$ & $\left(n_{2}+2\right) / 2$ & $\frac{m_{1}}{n_{1}}-\frac{1}{n_{2}}<\frac{1}{2}$ & $m_{1}-1$ \\
\hline$X_{0}^{p \boxminus}$ & $\left(n_{1}-1\right) / 3 \rightarrow\left(n_{1}-2\right) / 2$ & $n_{2}-2$ & $\frac{m_{1}}{n_{1}}>\frac{2}{n_{2}}$ & $n_{1}-2 m_{1}-1$ \\
\hline$X_{0}^{p b}$ & $\left(n_{1}-1\right) / 4 \rightarrow\left(n_{1}-2\right) / 3$ & $n_{2}-1$ & $\frac{m_{1}}{n_{1}}>\frac{1}{n_{2}}$ & $n_{1}-3 m_{1}-1$ \\
\hline$X_{1}^{\text {\#}}$ & $2\left(n_{1}+1\right) / 3$ & 1 & $\frac{2}{n_{1}}+\frac{3}{n_{2}}<1$ & 1 \\
\hline
\end{tabular}

(2) $m_{1}=1 ; m_{2}=n_{2}-2$ or $2\left(n_{2}+1\right) / 3$. They are also $(2,3 ; \mathrm{a})$.

(3) $m_{1}=1 ; n_{2}-3 \geq m_{2}>2\left(n_{2}+1\right) / 3, m_{2} \neq 3 n_{2} / 4 . \quad L(f) \leq 3$.

In general, following four types listed in Table 4.5 are case $(2,3$; a). $(\mathfrak{a}+\mathcal{O} f): a=\left(x^{a^{\prime}}, y\right)$

Table 4.5 i) 
Same notations are used when we exchange $x$ and $y$ and subscripts 1 and 2 .

The ideal $\mathfrak{a}: f$ and $a(x)=x^{k_{1}} y^{k_{2}}$ are listed up in the following.

Table 4.5 ii)

\begin{tabular}{c|c|c|c} 
& $\mathfrak{a}: f$ & $k_{1}$ & $k_{2}$ \\
\hline$S \# \#$ & $x^{m_{1}-1} y, y^{\left(n_{2}-2\right) / 2}-m_{2} t x^{m_{1}}$ & $n_{1}-m_{1}$ & $\left(n_{2}-2\right) / 2$ \\
$X_{0}^{\prime} \boxminus$ & $x^{n_{1}-2 m_{1}-1} y, y^{2}-m_{2} t x^{m_{1}}$ & $2 m_{1}$ & $n_{2}-4$ \\
$X_{0} \triangleright$ & $x^{n_{1}-2 m_{1}-1}, y-m_{2} t x^{m_{1}}$ & $3 m_{1}$ & $n_{2}-3$ \\
$X_{1}^{\# \cdot}$ & $x^{\left(n_{1}+1\right) / 3}, x^{\left(n_{1}-2\right) / 3}-m_{1} t y$ & $m_{1}-2$ & $n_{2}-2$ \\
\hline
\end{tabular}

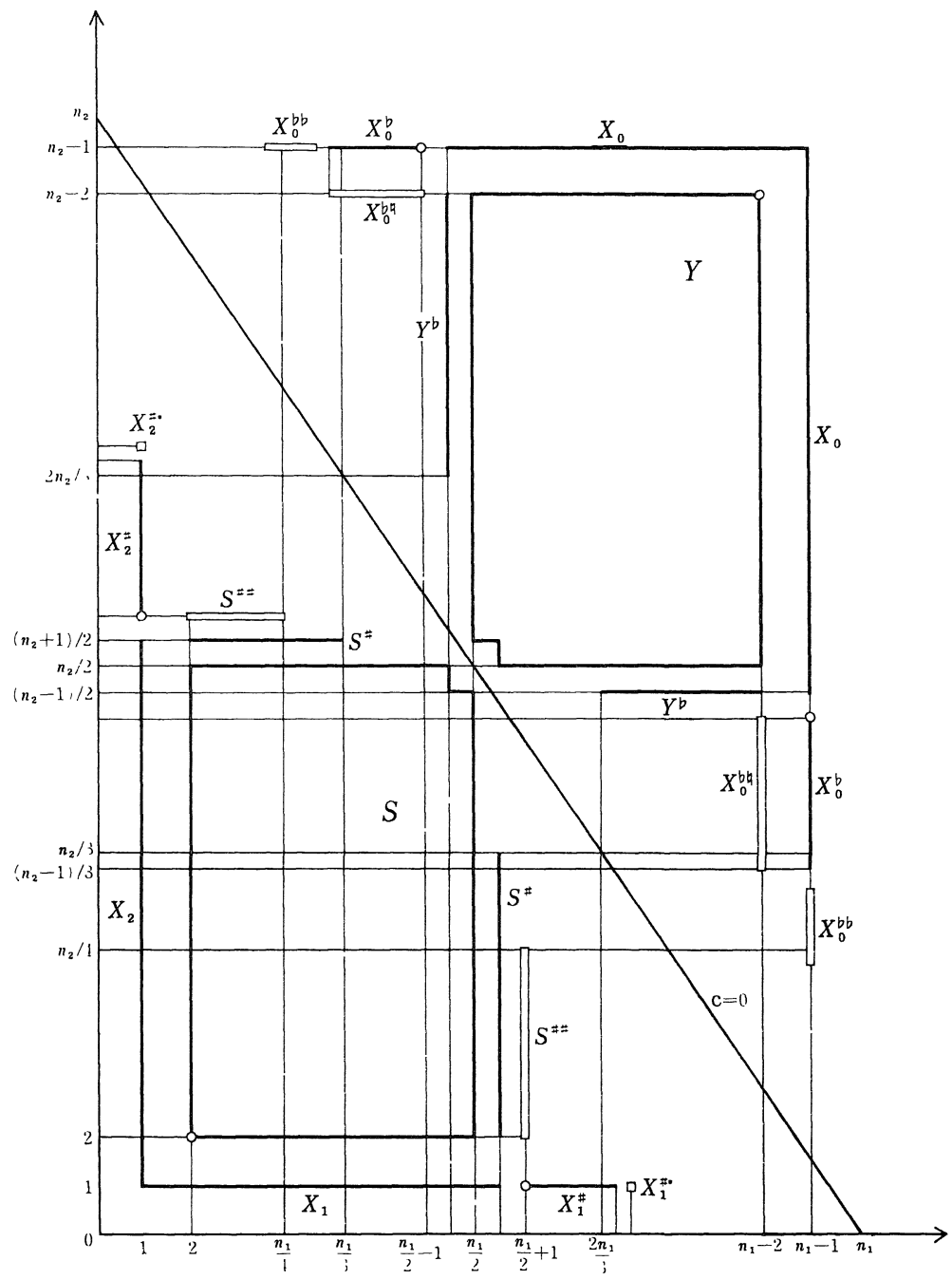

Fig. 4.6 
The structure of $g(s)$ in these four types and the determination of $b(s)$ is found in [32].

Note that Example 2.29 is of type $X_{0}^{\natural \triangleright}$, and Example 4.1 is also of type $X_{0}^{p b}$ if we performe the coördinate transform $X=x, Y=y$ $-\left(n_{2}-1\right) t x^{m_{1}}$.

The followings are proved to be $L(f) \leq 3$. We conjecture that equality holds.

$$
\begin{aligned}
& \left(n_{1}+2\right) / 2 \leq m_{1} \leq 2 n_{1} / 3, \quad 2 \leq m_{2} \leq n_{2} / 3 . \quad \text { e.g. } S^{\# \#} . \\
& 2 n_{1} / 3 \leq m_{1} \leq n_{1}-2, \quad n_{2} / 3 \leq m_{2} \leq\left(n_{1}-2\right) / 2 . \quad \text { e.g. } X_{0}^{p ᄆ} .
\end{aligned}
$$

$\mathrm{a}: f$ is given by $\left(x^{2 m_{1}-n_{1}-1} y^{m_{2}-1}, x^{n_{1}-m_{1}}-m_{1} t y^{m_{2}}\right)$ and $\left(x^{n_{1}-m_{1}-1} y^{n_{2}-2 m_{2}-1}\right.$, $\left.x^{n_{1}-m_{1}}-m_{1} t y^{m_{2}}\right)$ respectively.

Theorem 4. 7. When a: $f=\left(x^{a}, y^{b}\right), " E E F^{\prime \prime}$ holds for $f$. Parameters are listed in the following table. Moreover, we can choose second order operators as indicated in (3) and (4).

Type $1^{\circ}$.

$\begin{array}{lcccc} & \alpha & \beta & a & b \\ Y, Y^{\triangleright} & 1 / n_{1} & 1 / n_{2} & n_{1}-m_{1}-1 & n_{2}-m_{2}-1 \\ X_{0}^{b} & 1 / n_{1} & 1 / n_{2} & \left(\begin{array}{c}1 \\ n_{1}-2 m_{1}-1\end{array}\right. & n_{2}-2 m_{2}-1 \\ & & & 2 m_{1}-n_{1}-1 & 1 \\ X_{1}^{\#} & \left(n_{2}-m_{2}\right) / m_{1} n_{2} & 1 / n_{2} & 1 \\ X_{2}^{\#} & 1 / n_{1} & \left(n_{1}-m_{1}\right) / m_{2} n_{1} & 1 & 2 m_{2}-n_{2}-1\end{array}$

Type $2^{\circ}$.

$S, S^{\#}$

$$
\begin{aligned}
& a=m_{1}-1, \quad b=m_{2}-1 . \\
& A=\left(\begin{array}{cc}
\frac{n_{2}-m_{2}}{m_{1} n_{2}} & \frac{1}{n_{2}} \\
\frac{1}{n_{1}} & \frac{n_{1}-m_{1}}{m_{2} n_{1}}
\end{array}\right) .
\end{aligned}
$$

Proof. Generators of $g(s)$ were already discussed. In order to prove that $\tilde{b}(s)$ is given by "EEF", we determine eigenvectors and root vectors of $s$ in $\operatorname{Ham}_{\mathscr{D}}\left(\widetilde{\mathfrak{M}}, \mathscr{B}_{p t}\right)$ explicitly. Here, we performe this procedure taking as examples type $Y$ and $S$. Calculation for other types can be given similarly. 
We use the following notation:

$$
\begin{aligned}
& (a)_{b}=a(a-1) \cdots(a-b+1), \\
& {[a]_{b}=a(a+1) \cdots(a+b-1) .} \\
& (p(s))_{\text {red }}=\prod\left(s+\alpha_{i}\right), \text { for a polynomial } p(s)=\prod\left(s+\alpha_{i}\right)^{\varepsilon_{i}}, \quad \varepsilon_{i} \neq 0, \\
& a_{i} \neq \alpha_{j}, i \neq j .
\end{aligned}
$$

For the $\delta$-functions, we use the notation

$$
\delta[i, j]=D_{x}^{i} D_{y}^{j} \hat{\partial}(x, y) .
$$

Since indices $i$ and $j$ are complicated, we do not adopt the usual notation $\delta^{(i, j)}$.

$Y:$ The generators of $g(s)$ are

$$
\begin{aligned}
& x^{n_{1}-m_{1}-1}\left\{\left(s-X_{0}\right)-m_{1} m_{2} t^{2} x^{k_{1}} y^{k_{2}}\left(s-X_{2}\right)\right\}-c t y^{m_{2}} D_{x}, \\
& y^{n_{2}-m_{2}-1}\left\{\left(s-X_{0}\right)-m_{1} m_{2} t^{2} x^{k_{1}} y^{k_{2}}\left(s-X_{1}\right)\right\}-c t x^{m_{1}} D_{y}, \\
& \left(s-X_{0}+c\right)\left(s-X_{0}\right)-m_{1} m_{2} t^{2} \cdot x^{k_{1}} y^{k_{2}}\left(s-X_{1}\right)\left(s-X_{2}\right), \\
& k_{1}=2 m_{1}-n_{1}, \quad k_{2}=2 m_{2}-n_{2} .
\end{aligned}
$$

follows.

We set $s_{i j}=\frac{i+1}{n_{1}}+\frac{j+1}{n_{2}}$. Define delta functions $\Delta_{i j}, \Delta_{i j}^{\prime}$ and $u_{i j}$ as

$$
\begin{aligned}
& \Delta_{i j}=\delta[i, j]+\sum_{l \geq 1} c_{i, j}^{l} \delta\left[i-l k_{1}, j-l k_{2}\right] \\
& c_{i, j}^{l}= \frac{(-)^{l\left(k_{1}+k_{2}\right)}}{(2 l) !}\left(n_{1} n_{2} t^{2}\right)^{l}\left[\frac{i+1}{n_{1}}\right]_{l}\left[\frac{j+1}{n_{2}}\right]_{l}(i)_{l k_{1}}(j)_{l k_{2}} . \\
& \Delta_{i j}^{\prime}= \delta[i, j]+\sum_{l \geq 1} c_{i, j}^{\prime l} \delta\left[i-l k_{1}, j-l k_{2}\right], \\
& c_{i, j}^{\prime l}= \frac{(-)^{l\left(k_{1}+k_{2}\right)}}{(2 l+1) !}\left(n_{1} n_{2} t^{2}\right)^{l}\left[\frac{i+m_{1}+1}{n_{1}}\right]_{l}\left[\frac{j+m_{2}+1}{n_{2}}\right]_{l}(i)_{l k_{1}}(j)_{l k_{2}} . \\
& u_{i, j}= \Delta_{i j}+(-)^{n_{1}+m_{1}+m_{2}} \frac{(j)_{m_{2}} t}{\left(i+n_{1}-m_{1}\right)_{n_{1}-m_{1}-1}} J_{i+n_{1}-m_{1}, j-m_{2}}^{\prime} \\
& \quad+(-)^{n_{2}+m_{1}+m_{2}} \frac{(i)_{m_{1}} t}{\left(j+n_{2}-m_{2}\right)_{n_{2}-m_{2}-1}} \Delta_{i-m_{1}, j+n_{2}-m_{2}}^{\prime} .
\end{aligned}
$$

Then, $u_{i j}$ where $\left(0 \leq i \leq n_{1}-2,0 \leq j \leq m_{2}-1\right)$ or $\left(0 \leq i \leq m_{1}-1\right.$, $\left.0 \leq j \leq n_{2}-2\right)$ are eigenvectors belonging to eigenvalue $-s_{i j}$.

When $\left(0 \leq i \leq n_{1}-m_{1}-2,0 \leq j \leq n_{2}-m_{2}-2\right), \quad \Delta_{i j}^{\prime}$ are also eigen- 
vectors belonging to $-s_{i j}-c$.

Therefore,

$$
\begin{aligned}
\widetilde{P}(t)= & \frac{\left(t^{1 / n_{1}}-t\right)\left(t^{1 / n_{2}}-t\right)}{\left(1-t^{1 / n_{1}}\right)\left(1-t^{1 / n_{2}}\right)}+t^{1-\left(n_{1}-m_{1}\right) / n_{1}-\left(n_{2}-m_{2}\right) / n_{2}} \\
& \times(1-t) \frac{\left(t^{1 / n_{1}}-t^{\left(n_{1}-m_{1}\right) / n_{1}}\right)\left(t^{1 / n_{2}}-t^{\left(n_{2}-m_{2}\right) / n_{2}}\right)}{\left(1-t^{1 / n_{1}}\right)\left(1-t^{1 / n_{2}}\right)} . \\
\tilde{b}(s)= & \left(\prod_{\substack{0 \leq i \leq n_{1}-2 \\
0 \leq j \leq m_{2}-1 \\
0 \leq i \leq m_{1}-1 \\
0 \leq j \leq n_{2}-2}}\left(s+s_{i j}\right)_{\substack{0 \leq i \leq n_{1}-m_{1}-2 \\
0 \leq j \leq n_{2}-m_{2}-2}}\left(s+s_{i j}+c\right)\right)_{\mathrm{red}} .
\end{aligned}
$$

$S:$ The generators of $g(s)$ are

$$
\begin{aligned}
& x^{m_{1}-1}\left\{\left(s-X_{2}\right)-\frac{1}{m_{1} m_{2} t^{2}} x^{h_{1}} y^{h_{2}}\left(s-X_{0}\right)\right\}+\frac{c}{m_{1} m_{2} t} y^{n_{2}-m_{2}} D_{x}, \\
& y^{m_{2}-1}\left\{\left(s-X_{1}\right)-\frac{1}{m_{1} m_{2} t^{2}} x^{h_{1}} y^{h_{2}}\left(s-X_{0}\right)\right\}+\frac{c}{m_{1} m_{2} t} x^{n_{1}-m_{1}} D_{y}, \\
& \left(s-X_{1}\right)\left(s-X_{2}\right)-\frac{1}{m_{1} m_{2} t^{2}} x^{h_{1}} y^{h_{2}}\left(s-X_{0}+c\right)\left(s-X_{0}\right), \\
& h_{1}=n_{1}-2 m_{1}, \quad h_{2}=n_{2}-2 m_{2} .
\end{aligned}
$$

Set

$$
\begin{aligned}
& s_{i j}^{1}=\frac{\left(n_{2}-m_{2}\right)(i+1)+m_{1}(j+1)}{m_{1} n_{2}}, \\
& s_{i j}^{2}=\frac{m_{2}(i+1)+\left(n_{1}-m_{1}\right)(j+1)}{m_{2} n_{1}} .
\end{aligned}
$$

$d=$ g.c.d. $\left(m_{1}, m_{2}\right), \quad m_{1}=d m_{1}^{\prime}, \quad m_{2}=d m_{2}^{\prime} . \quad s_{i j}^{1} \quad$ and $s_{i j}^{2} \quad$ coincide for $i=r m_{1}^{\prime}-1, j=r m_{2}^{\prime}-1,1 \leq r \leq d$. We denote this value $r / d$ as $s_{r}$.

We make use of the following delta functions, $0 \leq i \leq n_{1}-2$, $0 \leq j \leq n_{2}-2$.

$$
\begin{aligned}
& \Delta_{i j}^{1}=\delta[i, j]+\sum_{l \geq 1} c_{i, j}^{l, 1} \delta\left[i-l h_{1}, j-l h_{2}\right] \\
& c_{i, j}^{l, 1}=\frac{(i)_{l h_{1}}(j)_{l h_{2}}(-)^{l\left(h_{1}+h_{2}\right)}\left[\frac{i+1}{m_{1}}\right]_{2 l}}{\left(n_{1} n_{2} t^{2}\right)^{l} l !\left[d_{i j}^{1}+1\right]_{l}} \\
& d_{i j}^{1}=\frac{m_{2}}{n_{2}}\left(\frac{i+1}{m_{1}}-\frac{j+1}{m_{2}}\right) .
\end{aligned}
$$


Similarly, we define $\Delta_{i j}^{2}$ by exchanging $i$ and $j$ and subscripts 1 and 2 in the above formulae. Since $d_{i, j}^{1}+1=\frac{m_{2}}{n_{2}}\left(\frac{i+1}{m_{1}}+\frac{n_{2}-j-1}{m_{2}}\right)$ and $d_{i, j}^{2}+1=\frac{m_{1}}{n_{1}}\left(\frac{n_{1}-i-1}{m_{1}}+\frac{j+1}{m_{2}}\right)$ are positive when $0 \leq i \leq n_{1}-2,0 \leq j \leq n_{2}$ -2 , they are well-defined.

Then,

$$
\Delta_{r}=\Delta_{r m_{1}^{\prime}-1, r m_{2^{\prime}-1}}^{1}=\Delta_{r m_{2^{\prime}-1, r m_{2^{\prime}-1}}^{2}, \quad 1 \leq r \leq d}^{2}
$$

are eigenfunction belonging to eigenvalue $-s_{r}$.

For other values of $(i, j)$, we set

$$
u_{i j}^{1}=\Delta_{i j}^{1}+\frac{(j)_{n_{2}-m_{2}}(-)^{n_{2}+m_{1}+m_{2}}}{m_{1} n_{2} t d_{i j}^{1}\left(i+m_{1}\right)_{m_{1}-1}} \Delta_{i+m_{1}, j-n_{2}+m_{2}}^{1},
$$

for $0 \leq i \leq n_{1}-2,0 \leq j \leq n_{2}-2$, and also set $u_{\imath j}^{2}$ for $0 \leq i \leq n_{1}-2,0 \leq j$ $\leq m_{2}-2$, in a usual manner.

Then, $u_{i j}^{1}$ and $u_{i j}^{2}$ are eigenvectors belonging to $-s_{i j}^{1}$ and $-s_{i j}^{2}$.

When $d \geq 2$, non-semisimple roots appear. We define the series $e_{t}^{l}$ by the following recursion formula.

$$
\left\{\begin{aligned}
e_{r}^{0}= & 0 \\
e_{r}^{l}= & \frac{1}{n_{1} n_{2} l^{2} t^{2}}\left(i-(l-1) h_{1}\right)_{h_{1}}\left(j-(l-1) h_{2}\right)_{h_{2}}(-)^{h_{1}+h_{2}} \\
& \times\left(\frac{r}{d}+2 l-2\right)\left(\frac{r}{d}+2 l-1\right) e_{r}^{l-1} \\
& -\frac{1}{c}\left\{\frac{c+1}{l}-\left(\frac{1}{\frac{r}{d}+2 l-2}+\frac{1}{\frac{r}{d}+2 l-1}\right)\right\} c_{r}^{l},
\end{aligned}\right.
$$

where $c_{r}^{l}=c_{r m_{1}^{\prime}-1, r m_{2}^{\prime}-1}^{l, 1}=c_{r m_{1}^{\prime}-1, r m_{2}^{\prime}-1}^{l, 2}$.

Set

$$
\begin{aligned}
& \Delta_{r}^{\prime}=\sum_{l=1} e_{r}^{l} \delta\left[r m_{1}^{\prime}-1-l h_{1}, r m_{2}^{\prime}-1-l h_{2}\right], \\
& \Delta_{r}^{\prime \prime}=\sum_{l \leq 0}\left(c_{r}^{l}-\frac{r}{d} e_{r}^{l}\right) \delta[", \quad " \quad] .
\end{aligned}
$$

Then, $u_{r}^{\prime}=\left[\begin{array}{c}\Delta_{r}^{\prime} \\ d_{r}^{\prime \prime}\end{array}\right]$ and $u_{r}=\left[\begin{array}{c}\Delta_{r} \\ -\frac{r}{d} \Delta_{r}\end{array}\right]$ form a root subspace belonging to eigenvalue $-\frac{r}{d}$ for $1 \leq r \leq d-1$. 


$$
s\left(u_{r}^{\prime}, u_{r}\right)=\left(u_{r}^{\prime}, u_{r}\right)\left[\begin{array}{cc}
-\frac{r}{d} & \\
1 & -\frac{r}{d}
\end{array}\right] .
$$

Thus, $b$-functions are determined.

$$
\begin{gathered}
\tilde{b}(s)=\prod_{r=1}^{d-1}\left(s+\frac{r}{d}\right)\left(\prod_{\substack{0 \leq i \leq m_{11}-2 \\
0 \leq j \leq n_{2}-2}}\left(s+s_{i j}^{1}\right)_{\substack{0 \leq i \leq n_{1}-2 \\
0 \leq j \leq m_{2}-2}}\left(s+s_{i j}^{2}\right)(s+1)\right)_{\mathrm{red}}, \\
\widetilde{P}(t)=t+\frac{t^{a_{11}+a_{12}}(1-t)\left(1-t^{\left(m_{1}-1\right) a_{11}}\right)}{\left(1-t^{a_{11}}\right)\left(1-t^{a_{12}}\right)}+\frac{\left.t^{a_{21}+a_{22}}(1-t)\left(1-t^{\left(m_{2}-1\right.}\right)^{a_{22}}\right)}{\left(1-t^{a_{21}}\right)\left(1-t^{a_{22}}\right)}, \\
{\left[\begin{array}{ll}
a_{11} & a_{12} \\
a_{21} & a_{22}
\end{array}\right]=\left[\begin{array}{cc}
\frac{n_{2}-m_{2}}{m_{1} n_{2}} & \frac{1}{n_{2}} \\
\frac{1}{n_{1}} & \frac{n_{1}-m_{1}}{m_{2} n_{1}}
\end{array}\right] .} \\
\chi(\tau)=\frac{\left(\tau^{m_{2} n_{1}}-1\right)\left(\tau^{m_{1} n_{2}}-1\right)}{\left(\tau^{n_{1}}-1\right)\left(\tau^{n_{2}}-1\right)}(\tau-1)
\end{gathered}
$$

is the characteristic polynomial of the local monodromy of $f^{-1}(0)$, when g.c.d. $\left(\left(n_{1}-m_{1}\right), m_{2}\right)=$ g.c.d. $\left(m_{1},\left(n_{2}-m_{2}\right)\right)=1$.

\section{§ 19. Other Examples}

In this section, we show four examples in 2-dimensional case and two examples in 3-dimensional case. Interesting examples in 3-dimension can be found in [32].

Reduced quasi-homogeneous polynomial in 2-variables is essentially one of the following three types.

$$
x^{n_{1}}+y^{n_{2}}, \quad x\left(x^{n_{1}}+y^{n_{2}}\right), \quad x y\left(x^{n_{1}}+y^{n_{2}}\right) .
$$

In $\S 18$, we investigated non-quasi-homogeneous functions derived from the first type. Examples $4.8 \sim 11$ are dealt with those derived from the second and the third type. In this section, we restrict ourselves to the most typical classes, that is, those similar to $Y$ and $S$ in $\S 18$. It should be noted that one can take

$$
\left(x^{n_{1}-m_{1}}+y^{m_{2}}\right)\left(x^{m_{1}}+y^{n_{2}-m_{2}}\right)
$$

instead of type $S$ owing to Proposition 2.12. We adopt this form in 
Examples $x S$ and $x y S$.

We exhibit generators of $g(s)$, and values of parameters in "EEF" in Table 4.12. The determination of the action of $s$ for these examples is similar to that for type $Y$ and $S$ and we omit the details.

Example 4. 8. $x Y$

$$
\begin{gathered}
f=x\left(\frac{1}{n_{1}} x^{n_{1}}+\frac{1}{n_{2}} y^{n_{2}}-x^{m_{1}} y^{m_{2}}\right) . \\
n_{1} / 2 \leq m_{1} \leq n_{1}-2, \quad n_{2} / 2 \leq m_{2} \leq n_{2}-1 .
\end{gathered}
$$

In this case,

$$
\mu=\left(n_{1}+1\right)\left(n_{2}-1\right)+1
$$

Set

$$
\begin{aligned}
& c=\left(m_{1} m_{2}-\left(n_{1}-m_{1}\right)\left(n_{2}-m_{2}\right)\right) /\left(n_{1}+1\right) n_{2}, \\
& k=\left(\left(m_{1}+1\right) n_{2}-m_{2}\right) / n_{2}, \\
& k^{\prime}=\left\{\left(m_{1}+1\right)^{2} n_{2}+m_{2}^{2}-\left(m_{1}+1\right) m_{2} n_{2}\right\} / n_{2} m_{2}^{2}, \\
& \varphi=1-k x^{2 m_{1}-n_{1}} y^{2 m_{2}-n_{2}},
\end{aligned}
$$

and

$$
X_{0}=\left(n_{2} x D_{x}+n_{2} y D_{y}\right) /\left(n_{1}+1\right) n_{2} .
$$

$g(s)$ is generated by

$$
\begin{aligned}
& x^{n_{1}-m_{1}}\left(s-X_{0}\right)-\frac{c}{\varphi}\left\{m_{2} x^{2 m_{1}-n_{1}+1} y^{m_{2}-1} D_{x}+\left(x^{m_{1}}+k x y^{2 n_{2}-m_{2}-1}\right) D_{y}\right\} \\
& y^{n_{2}-m_{2}-1}\left(s-X_{0}\right)-\frac{c}{\varphi} y^{n_{2}}\left\{x y^{n_{2}-m_{2}-1} D_{x}-\left(k x^{n_{1}-1}+k^{\prime} y^{n_{2}-m_{2}}\right) D_{y}\right\}
\end{aligned}
$$

and

$$
\begin{aligned}
\left(s-X_{0}+c\right)\left(s-X_{0}\right) & -\frac{k}{\varphi} x^{2 m_{1}-n_{1}} y^{2 m_{2}-n_{2}} \\
& \times\left\{\left(2 X_{0}-X_{1}-X_{2}-c\right) s+X_{1} X_{2}-X_{0}^{2}+X_{0} c\right\} .
\end{aligned}
$$

Example 4.9. $x y Y$

$$
f=x y\left(x^{n_{1}}+y^{n_{2}}-x^{m_{1}} y^{m_{2}}\right)
$$




$$
n_{1} / 2 \leq m_{1} \leq n_{1}-1, \quad n_{2} / 2 \leq m_{2} \leq n_{2}-1, \quad \mu=\left(n_{1}+1\right)\left(n_{2}+1\right) .
$$

Set

$$
c=\left\{m_{1} m_{2}-\left(n_{1}-m_{1}\right)\left(n_{2}-m_{2}\right)\right\} /\left\{\left(n_{1}+1\right)\left(n_{2}+1\right)-1\right\}
$$

and

$$
X_{0}=\left(n_{2} x D_{x}+n_{1} y D_{y}\right) /\left\{\left(n_{1}+1\right)\left(n_{2}+1\right)-1\right\} .
$$

$\mathscr{g}(s)$ is generated by

$$
\begin{aligned}
& x^{n_{1}-m_{1}}\left(s-X_{0}\right)+\frac{c}{c_{1}} y^{m_{2}}\left(s-X_{1}\right), \\
& y^{n_{2}-m_{2}}\left(s-X_{0}\right)+\frac{c}{c_{2}} x^{m_{1}}\left(s-X_{2}\right),
\end{aligned}
$$

and

$$
\left(s-X_{0}+c\right)\left(s-X_{0}\right)-\frac{c^{2}}{c_{1} c_{2}} x^{2 m_{1}-n_{1}} y^{2 m_{2}-n_{2}}\left(s-X_{1}\right)\left(s-X_{2}\right) .
$$

Example 4. 10. $x S$

$$
f=x\left(x^{m}+y^{n}\right)\left(x^{\mu}+y^{\nu}\right), \quad \mu \geq m, n \geq \nu .
$$

Milnor $=(m+1)(n+\nu)+(\nu-1)(m+\mu)$.

Set

$$
\begin{aligned}
& c=n /-\nu m, \quad m^{\prime}=m+\mu+1, \quad k=\frac{n(\mu(n+\nu)+\nu)}{\nu(m(n+\nu)+n)} . \\
& \Theta=\nu x D_{x}+\mu y D_{y}, \quad \Psi=n x D_{x}+m y D_{y}, \\
& X_{1}=\Psi /\{m(n+\nu)+n\}, \quad X_{2}=\Theta /\{\mu(n+\nu)+\nu\} . \\
& Y_{1}=\Theta / \nu m^{\prime}, \quad Y_{2}=\Psi / n m^{\prime} .
\end{aligned}
$$

$g(s)$ is generated by

$$
\begin{aligned}
& x^{m}\left(s-Y_{1}\right)-\frac{c y^{n}}{m m^{\prime} \nu}\left(s-x D_{x}\right)-\frac{n / \ell}{m \nu} x^{\mu} y^{n-\nu}\left(s-X_{1}\right) \\
& y^{\nu-1}\left(s-X_{1}\right)-k x^{\mu-m} y^{n-\nu}\left(s-Y_{1}\right)+\frac{c x^{\mu}}{\nu(m(n+\nu)+n)} D_{y},
\end{aligned}
$$

and

$$
\left(s-X_{1}\right)\left(s-Y_{1}\right)-k x^{\mu-m} y^{n-\nu}\left(s-X_{2}\right)\left(s-Y_{2}\right) .
$$


Example 4. 11. $x y S$

$$
f=x y\left(x^{m}+y^{n}\right)\left(x^{\mu}+y^{\nu}\right), \mu \geq m, n \geq \nu .
$$

Milnor $=(n+1)(n+\nu)+(\nu+1)(m+\mu)+1$.

Notations $c, \Theta$ and $\Psi$ are same as in 4.10. Set

$$
\begin{aligned}
& c^{\prime}=(m+\mu+1)(n+\nu+1)-1, \\
& k_{X_{1}}=m(n+\nu)+m+n, \quad k_{X_{2}}=\mu(n+\nu)+\mu+\nu . \\
& k_{Y_{1}}=\nu(\mu+m)+\mu+\nu, \quad k_{Y_{2}}=n(m+\mu)+m+n, \\
& X_{1}=\Psi / k_{X_{1}}, X_{2}=\Theta / k_{X_{2}}, Y_{1}=\Theta / k_{Y_{1}}, Y_{2}=\Psi / k_{Y_{2}} .
\end{aligned}
$$

$g(s)$ is generated by

$$
\begin{aligned}
& x^{m}\left(s-Y_{1}\right)+\frac{k_{X_{2}}}{k_{Y_{1}}} y^{n}\left(s-X_{2}\right), \\
& y^{\nu}\left(s-X_{1}\right)+\frac{k_{X_{2}}}{k_{X_{1}}} x^{\mu}\left(s-Y_{2}\right),
\end{aligned}
$$

and

$$
\left(s-X_{1}\right)\left(s-Y_{1}\right)-\frac{k_{X_{2}} k_{Y_{2}}}{k_{X_{1}} k_{Y_{1}}} x^{\mu-m} y^{n-\nu}\left(s-X_{2}\right)\left(s-Y_{2}\right)
$$

One can apply "EEF" for preceding four examples by setting parameters in the following Table

Table 4.12

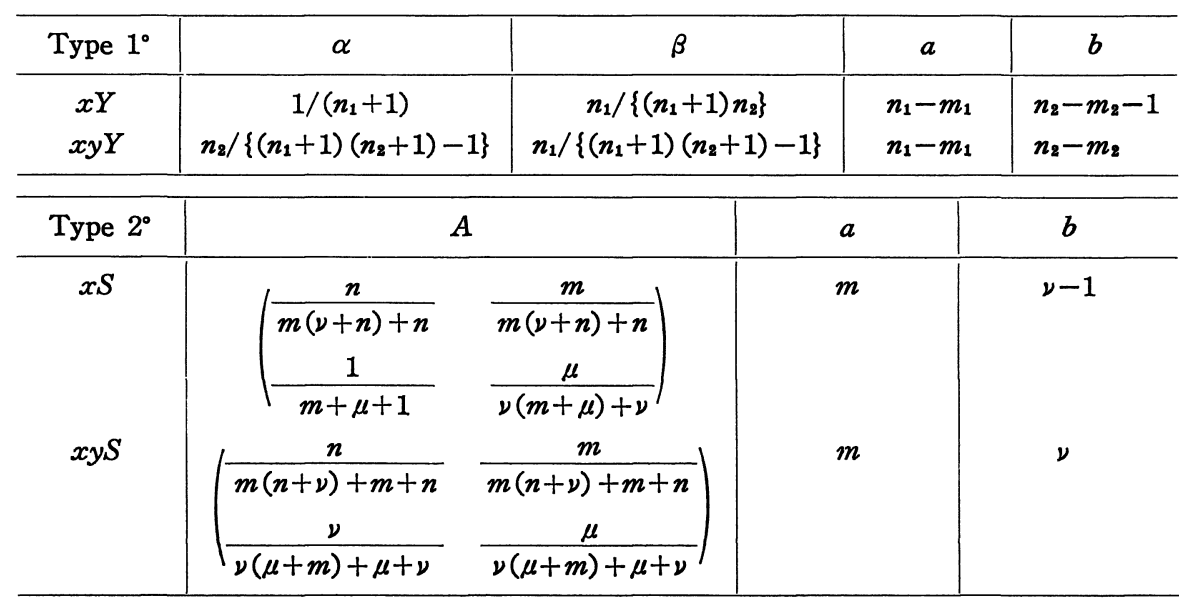


Example 4. 13.

$$
\begin{aligned}
& T_{p, q, r}: \quad f=\frac{1}{p} x^{p}+\frac{1}{q} y^{q}+\frac{1}{r} z^{r}-a x y \approx \\
& \mu=p+q+r-1 . \quad c=\frac{1}{p}+\frac{1}{q}+\frac{1}{r}-1, \quad a: \text { parameter } \\
& X_{0}=\frac{1}{p} x D_{x}+\frac{1}{q} y D_{y}+\frac{1}{r} z D_{z}, \quad X=\frac{1}{3}\left(x D_{x}+y D_{y}+z D_{z}\right) \\
& X_{1}=X_{0}-c x D_{x}, \quad X_{2}=X_{0}-c y D_{y}, \quad X_{3}=X_{0}-c z D_{z} . \\
& X_{210}=\frac{-1}{\varphi}\left(y^{q-2} z^{r-3} D_{x}+a z^{r-2} D_{y}+a^{2} x D_{z}\right),
\end{aligned}
$$

and $X_{120}$ etc. are defined by permutation of variables and $\{p, q, r\}$.

$$
\begin{aligned}
Q=\left(\frac{1}{3}-\frac{1}{p}\right) x^{p-3} X_{201} \cdot X_{210} & +\left(\frac{1}{3}-\frac{1}{q}\right) y^{q-3} X_{120} \cdot X_{021} \\
& +\left(\frac{1}{3}-\frac{1}{r}\right) z^{r-3} X_{012} \cdot X_{102}
\end{aligned}
$$

$\varphi=a^{3}-x^{p-3} y^{q-3} z^{r-3}$

The generators of $\mathscr{g}(s)$ are $X_{i j}$ 's and

$$
\begin{aligned}
& y\left(s-X_{1}\right)-c x^{p-2} X_{210}, z\left(s-X_{2}\right)-c y^{q-2} X_{021}, x\left(s-X_{3}\right)-c z^{r-2} X_{102}, \\
& \left(s-X_{0}+c\right)(s-X)-\frac{2 c}{\varphi} x^{p-3} y^{q-3} z^{r-3}\left(s-\frac{1}{2}\left(X_{0}+X\right)\right)+a c Q,
\end{aligned}
$$

or

$$
\begin{aligned}
\left(s-X_{1}\right)\left(s-X_{2}\right) & -c^{2} x^{p-3} y^{q-3} X_{210} \cdot X_{120} \\
& -\frac{c x^{p-3} y^{q-3} z^{r-3}}{\varphi}\left(3 s-X_{2}+2 X_{0}\right), \text { etc. }
\end{aligned}
$$

$\left(\begin{array}{c}\delta \\ -\delta\end{array}\right)$ and $\left(\begin{array}{l}0 \\ \delta\end{array}\right)$ forms a root subspace belonging to eigenvalue -1

Other eigenvectors are

$$
\begin{aligned}
& \Delta_{\nu}^{(1)}=D_{x}^{\nu} \delta, \quad 1 \leq \nu \leq p-2 \leftrightarrow-1-\frac{\nu}{p} \\
& \Delta_{p-1}^{(1)}=\left(D_{x}^{p-1}+\frac{(p-1) !}{a}(-)^{p-1} D_{y} D_{z}\right) \delta \leftrightarrow-1-\frac{p-1}{p},
\end{aligned}
$$


and similar delta functions by exchanging $x, y, z$.

$$
\tilde{b}(s)=(s+1)^{2}\left(\prod_{\substack{1 \leq \nu \leq p-1 \\ 1 \leq \nu, \leq q-1 \\ 1 \leq \nu^{\prime \prime} \leq r-1}}\left(s+1+\frac{\nu}{p}\right)\left(s+1+\frac{\nu^{\prime}}{q}\right)\left(s+1+\frac{\nu^{\prime \prime}}{r}\right)\right)_{\mathrm{red}} .
$$

It is remarkable that

$$
\widetilde{P}(t)=-t+t(1-t)\left(\frac{1}{1-t^{1 / p}}+\frac{1}{1-t^{1 / q}}+\frac{1}{1-t^{1 / r}}\right)
$$

and exponents $(1 / p, 1 / q, 1 / r)$ can be found through the coefficients of $X_{1}, X_{2}, X_{3}$ in the following manner.

$$
\begin{aligned}
& {\left[\begin{array}{ccc}
1-\frac{1}{q}-\frac{1}{r} & \frac{1}{q} & \frac{1}{r} \\
\frac{1}{p} & 1-\frac{1}{p}-\frac{1}{r} & \frac{1}{r} \\
\frac{1}{p} & \frac{1}{q} & 1-\frac{1}{p}-\frac{1}{q}
\end{array}\right)=\left(1-\frac{1}{p}-\frac{1}{q}-\frac{1}{r}\right) I_{3}} \\
& +\left(\begin{array}{l}
1 \\
1 \\
1
\end{array}\right)(1 / p, 1 / q, 1 / r) .
\end{aligned}
$$

This is similar to "EEF" type $2^{\circ}$.

Example 4. 14. $f=\frac{1}{3} x^{3}+\frac{1}{3} y^{3}+\frac{1}{4} z^{4}-a x y z^{2} . \quad a$ : parameter. $\mu=12, c=1 / 6$. Set

$$
\begin{aligned}
& X_{0}=\frac{1}{3} x D_{x}+\frac{1}{3} y D_{y}+\frac{1}{4} z D_{z}, \varphi=1-2 a^{3} z^{2} \\
& Q_{1}=\frac{a}{\varphi}\left(\frac{z}{a} D_{x}+2 a x z D_{y}+y D_{z}\right), Q_{2}=\frac{a}{\varphi}\left(2 a y z D_{x}+\frac{z}{a} D_{y}+x D_{z}\right) . \\
& Q=Q_{1} Q_{2}+Q_{2} Q_{1} .
\end{aligned}
$$

$\bar{y}(s)$ is generated by $X_{i j}$ and

$$
\begin{aligned}
& x\left(s-X_{0}\right)-\frac{a}{6 \varphi} z^{2}\left(y D_{x}+a z^{2} D_{y}+a^{2} x z D_{z}\right) \\
& y\left(s-X_{0}\right)-\frac{a}{6 \varphi} z^{2}\left(a z^{2} D_{x}+x D_{y}+a^{2} y z D_{z}\right)
\end{aligned}
$$




$$
\begin{aligned}
& \approx\left(s-X_{0}\right)-\frac{a}{6 \varphi}\left\{\left(a x z^{2}+y^{2}\right) a \approx D_{x}+\left(a y z^{2}+x^{2}\right) a \approx D_{y}+x y D_{z}\right\} \\
& \left(s-X_{0}+c\right)\left(s-X_{0}\right)+\frac{a^{2} z^{2}}{6^{2} \varphi}\left(5 s+\frac{11}{6}\left(x D_{x}+y D_{y}\right)+\frac{3}{2} z D_{z}\right)-\frac{a^{2} z^{2}}{12} Q .
\end{aligned}
$$

The correspondence of eigenvectors and eigenvalues are as follows. (We omit the minus sign).

$$
\begin{aligned}
& \left.\delta \leftrightarrow \frac{11}{12}, \frac{13}{12}, D_{x} \delta, D_{y} \delta \leftrightarrow \frac{5}{4}, D_{x} D_{y} \hat{0} \leftrightarrow \frac{19}{12}, \begin{array}{l}
\left(D_{y} D_{z}^{2}-a D_{x}^{2}\right) \delta \\
\left(D_{x} D_{z}^{2}-a D_{y}^{2}\right) \delta
\end{array}\right) \frac{7}{4}, \\
& D_{z} \delta \leftrightarrow \frac{7}{6}, \quad \begin{array}{l}
D_{y} D_{z} \delta \\
D_{x} D_{z} \delta
\end{array} \leftrightarrow \frac{3}{2}, D_{z}^{2} \hat{\jmath} \leftrightarrow \frac{17}{12}, \quad\left(D_{x} D_{y} D_{z}+\frac{a}{3} D_{z}^{3}\right) \delta \leftrightarrow \frac{13}{12},
\end{aligned}
$$

Thus $b$-function is determined as follows.

$$
\begin{aligned}
\tilde{b}(s) & =\left(s+\frac{3}{2}\right)\left(s+\frac{5}{4}\right)\left(s+\frac{7}{4}\right)\left(s+\frac{7}{6}\right)\left(s+\frac{11}{6}\right) \\
& \times\left(s+\frac{11}{12}\right)\left(s+\frac{13}{12}\right)\left(s+\frac{17}{12}\right)\left(s+\frac{19}{12}\right) \\
\widetilde{P}(t) & =\frac{\left(t^{1 / 3}-t\right)\left(t^{1 / 3}-t\right)\left(t^{1 / 4}-t\right)}{\left(1-t^{1 / 3}\right)\left(1-t^{1 / 3}\right)\left(1-t^{1 / 4}\right)}+t^{2-1 / 3-1 / 3-1 / 4}(1-t) .
\end{aligned}
$$

In view of this, $\tilde{P}(t)$ is given by "EEF" type $1_{(3)}$.

\section{$\S$ 20. Remarks on the Canonical Forms of Isolated Singularities}

According to V. I. Arnold, isolated singularities with modality not greater than two are completely classified, up to stable equivalence, by the following lists [2], [5].

1. 0-modal case

$$
A_{k}, D_{k}, E_{6}, E_{7}, E_{8}
$$

2. 1-modal case (with parameter a)

(1) $P_{8}, X_{9}, J_{10}$.

(2) $P_{p+5}, R_{p, q}, T_{p, q, r}, X_{p+5}, Y_{p, q}, J_{p+4}$

(3) 14 exceptional families.

3. 2-modal case (with parameters b and c or $\boldsymbol{a}=a_{0}+a_{1} y$ )

(1) $J_{3,0}, Z_{1,0}, W_{1,0}, Q_{2,0}, S_{1,0}, U_{1,0}$ 
(2) $J_{3, p}, Z_{1, p}, W_{1, p}, W_{1,2 q-1}^{\#}, W_{1,2 q}^{\#}, Q_{2, p}, S_{1, p}, S_{1,2 q-1}^{\#}, S_{1,2 q}^{\#}, U_{1,2 q-1}$, $U_{1,2 q}$.

(3) 14 exceptional families.*

The case 1 is weighted homogeneous. 2-(1) and 3-(1) $(c=0$ or $\left.a_{1}=0\right)$ are weighted homogeneous 1-parameter family. As for 2-(3) and 3-(3), they are weighted homogeneous when $a=0$ and $a_{0}=a_{1}=0$ respectively, and forms $\mu$-constant family of deformation. They are nonquasi-homogeneous and of simplex type, and hence if $a_{0} \neq 0$ in 3-(3), we can assume $a_{1}=0$ by Proposition 3.12 .

2-(2) and 3-(2) except $W_{1, p}^{\#}$ are also of simplex type, and we can assume $a_{1}=0$ in 3-(2), except $W_{1, p}^{\#}$.

Theorem 4. 15. In all the canonical forms with modality less than three, $L(f)=2$ holds if $f$ is non-quasi-homogeneous. $a: f$ is given as follows.

$$
\begin{array}{ll}
(x, y) \text { or }(x, y, z) & 2 \text {-(2, 2-(3) with } a \neq 0,3 \text {-(1) with } c \neq 0 \text { or } \\
& a_{1} \neq 0, \text { and 3-(3) with } a_{0}=0, a_{1} \neq 0 . \\
& \text { 3-(2, and 3-(3) with } a_{0} \neq 0 .
\end{array}
$$

We can determine the action of $s$ in $\operatorname{Ham}_{\mathscr{D}}\left(\widetilde{\mathscr{M}}, \mathscr{B}_{p t}\right)$ and know $\tilde{b}(s)$ and local monodromy. Especially,

1. $s$ is semisimple in cases 2-(3), 3-(2) and 3-(3).

2. In cases $P_{p+5}, R_{p, q}, T_{p, q, r}, \tilde{b}(s)$ has a double factor $(s+1)^{2}$, and in $X_{p+5}, Y_{p, q}, J_{p+4},\left(s+\frac{1}{2}\right)^{2}$.

3. In the cases of two variables except $W_{1, p}^{\#}$, and three variables in 2-(3) and 3-(3), "EEF" holds. Although $W_{1, p}^{\#}$ satisfies conditions (a) and (c) in $\S 17$, it does not satisfy (b).

As for the proof of this theorem, the author restrict himself to cases corank $(f)=2$ except $W_{1, p}^{\#}, J_{3,0}, Z_{1,0}$ and $W_{1,0}$. The proof for cases referred to above and $f$ being corank $(f)=3$ is included in [32].

* In V. I. Arnold's papers [4], [5] and [40], $y^{8}$ should be read $y^{11}$ in $E_{10}$ and $E_{20 .}$ 
Proof) In the next table, we give the correspondence between V. I. Arnold's classification and the author's. Most of those types have been already discussed. Example 4.19 below gives special types corresponding to Arnold's class $Z_{*}$ and $Z_{1, p}$.

Table 4.17 is concerned with classes in 3-(1), which are not of simplex type, together with more general classes $J_{k, 0}$ and $Z_{i, 0}$ and parameters appearing in "EEF". The detailed structure of them are included in [32], with the stuucture of $W_{1, p}^{\#}$.

Q.E.D.

Table 4.16

\begin{tabular}{|c|c|c|c|c|c|c|c|}
\hline 2-(2) & & $\alpha$ & $\beta$ & 3-(2) & & $\alpha$ & $\beta$ \\
\hline$J_{p+4}$ & $S_{\text {min }}^{\#}$ & $1 / 3$ & $1 / p$ & $J_{8, p}$ & $S \#$ & $1 / 3$ & $1 /(9+p)$ \\
\hline$X_{p+5}$ & $S_{\min }$ & $1 / 4$ & $1 / p$ & $W_{1, p}$ & $S$ & $1 / 4$ & $1 /(6+p)$ \\
\hline$Y_{p, q}$ & $S_{\min }$ & $1 / p$ & $1 / q$ & $Z_{1, p}$ & $y S^{\prime}$ & $\left(\begin{array}{l}(6+p) \\
3(7+p)\end{array}\right)$ & $1 /(7+p)$ \\
\hline 2-(3) & & $\alpha$ & $\beta$ & 3-(3) & & $\alpha$ & $\beta$ \\
\hline$E_{12}$ & $Y_{\max }^{b}$ & $1 / 3$ & $1 / 7$ & $E_{18}$ & $Y b$ & $1 / 3$ & $1 / 10$ \\
\hline$E_{18}$ & $X_{2, \min }^{\#}$ & $1 / 3$ & $2 / 15$ & $E_{10}$ & $X_{2}^{\#}$ & $1 / 3$ & $2 / 21$ \\
\hline$E_{14}$ & $Y_{\max }^{b}$ & $1 / 3$ & $1 / 8$ & $E_{20}$ & $Y b$ & $1 / 3$ & $1 / 11$ \\
\hline$Z_{11}$ & $y Y_{\max }^{b}$ & $4 / 15$ & $1 / 5$ & $Z_{17}$ & $y Y b$ & $7 / 24$ & $1 / 8$ \\
\hline$Z_{12}$ & $y X_{z, \min }^{\#}$ & $3 / 11$ & $2 / 11$ & $Z_{18}$ & $y X_{z}^{\#}$ & $5 / 17$ & $2 / 17$ \\
\hline$Z_{18}$ & $y Y_{\max }^{b}$ & $5 / 18$ & $1 / 6$ & $Z_{10}$ & $y Y b$ & $8 / 27$ & $1 / 9$ \\
\hline$W_{12}$ & $Y_{\max }$ & $1 / 4$ & $1 / 5$ & $W_{17}$ & $X_{z}^{\#}$ & $1 / 4$ & $3 / 20$ \\
\hline$W_{18}$ & $X_{z, \min }^{\#}$ & $1 / 4$ & $3 / 16$ & $W_{18}$ & $Y$ & $1 / 4$ & $1 / 7$ \\
\hline
\end{tabular}

Table 4.17

\begin{tabular}{c|c|c|c|c}
\hline $3-(1)$ & similar type & $\alpha$ & $\beta$ & $b$ \\
\hline$J_{\mathbf{8}, 0}$ & $Y_{\max }^{b}$ & $1 / 3$ & $1 / 9$ & 1 \\
$Z_{1,0}$ & $y Y_{\max }^{b}$ & $2 / 7$ & $1 / 7$ & 1 \\
$W_{1,0}$ & $Y_{\max }$ & $1 / 4$ & $1 / 6$ & 1 \\
$J_{k, 0}$ & $Y b$ & $1 / 3$ & $1 / 3 k$ & $k-2-d$ \\
$Z_{i, 0}$ & $y Y b$ & $(i+1) /(3 i+4)$ & $1 /(3 i+4)$ & $i-d$ \\
\hline
\end{tabular}

The number $d$ is determined by

$$
c \equiv 0 \bmod y^{d}, \quad \not \equiv 0 \bmod y^{d+1} \text {. }
$$

Among the Arnold's classification, following eight types in Table 4.18 are also of simplex type. $J_{k, i}$ and $E_{*}\left(Z_{i, p}\right.$ and $Z_{*}$, respectively) includes parameter $\boldsymbol{a}=a_{0}+\cdots+a_{k-2} y^{k-2} \quad\left(\boldsymbol{b}=b_{0}+\cdots+b_{i} y^{i}\right.$, respectively), 
and $a_{0} \neq 0\left(b_{0} \neq 0\right.$, respectively $)$ in $J_{k, i}\left(Z_{i, p}\right.$, respectively). $a: f=\left(x, y^{b}\right)$. The number $d$ in types $E_{*}$ and $Z_{*}$ is determined by

$$
\begin{aligned}
& a \equiv 0 \bmod y^{d}, \quad \not \equiv 0 \bmod y^{d+1}, \text { for } E_{*} . \\
& b \equiv 0 \bmod y^{d}, \quad \not \equiv 0 \bmod y^{d+1}, \text { for } Z_{*} .
\end{aligned}
$$

\begin{tabular}{|c|c|c|c|c|}
\hline & & $\alpha$ & $\beta$ & $\boldsymbol{b}$ \\
\hline$J_{k, i}$ & $S \#$ & $1 / 3$ & $1 /(3 k+i)$ & $k-1$ \\
\hline$Z_{i, p}$ & $y S \#$ & $(3 i+p+3) / 3(3 i+p+4)$ & $1 /(3 i+p+4)$ & $i+1$ \\
\hline$E_{0 k}$ & $Y b$ & $1 / 3$ & $1 /(3 k+1)$ & $k-1-d$ \\
\hline$E_{6 x+1}$ & $X_{2}^{\#}$ & $1 / 3$ & $2 / 3(2 k+1)$ & $k-1-d$ \\
\hline$E_{6 k+2}$ & $Y^{b}$ & $1 / 3$ & $1 /(3 k+2)$ & $k-1-d$ \\
\hline$Z_{6 i+11}$ & $y Y^{b}$ & $(3 i+4) / 3(3 i+5)$ & $1 /(3 i+5)$ & $i+1-d$ \\
\hline$Z_{8 i+12}$ & $y X_{z}^{\#}$ & $(2 i+3) /(6 i+11)$ & $2 /(6 i+11)$ & $i+1-d$ \\
\hline$Z_{B t+18}$ & $y Y^{b}$ & $(3 i+5) / 9(i+2)$ & $1 /(3 i+6)$ & $i+1-d$ \\
\hline
\end{tabular}

Table 4. 18

Operators and ideals $\mathfrak{a}: f$ for $Z_{i, p}$ and $Z_{*}$ are included in the following example $Z$.

\section{Example 4. 19.}

$Z: f=\frac{1}{3} x^{3} y+\frac{1}{n} y^{n}-t x^{m_{1}} y^{m}$

$$
120
$$

i) $m_{1}=1,2 m \geq n+2, n>m . \quad c=\frac{1}{3}\left(1-\frac{1}{n}\right)+\frac{m}{n}-1(\neq 0)$

$$
\begin{aligned}
& x\left(s-X_{2}\right)-\frac{3}{(3 m-1) t} y^{n-m}\left(s-X_{0}\right) \\
& y^{2 m-n-1}\left(s-X_{2}+\frac{3 c}{(3 m-1) t} y^{n-m} D_{x}\right)-\frac{3 x}{(3 m-1) t}\left(s-X_{0}\right)
\end{aligned}
$$

a) $2 n+1>3 m$ a $: f=\left(x, y^{2 m-n-1}\right)$ type $y X_{2}^{\#}, Z_{6 i+12}: \begin{aligned} & n=3 i+6+d \\ & m=2 i+4\end{aligned}$

$$
\begin{aligned}
\left(s-X_{2}-\frac{3 n c}{3 m-1}\right) & \left(s-X_{2}+\frac{3 c}{(3 m-1) t} y^{n-m} D_{x}\right) \\
& -\frac{1}{t}\left(\frac{3}{3 m-1}\right)^{2} y^{2 n-3 m+1}\left(s-X_{0}+c\right)\left(s-X_{0}\right)
\end{aligned}
$$


b) $2 n+1<3 m$ a $: f=\left(x, y^{n-m}\right)$ type $y Y^{b}, \begin{aligned} & Z_{6 i+11}: \begin{array}{l}n=3 i+5 \\ m=2 i+4+d\end{array} \\ & m=3 i+6\end{aligned}$ $Z_{6 i+13}: \begin{aligned} & n=3 i+6 \\ & m=2 i+5+d\end{aligned}$

$$
\begin{aligned}
\left(s-X_{0}+c\right)(s & \left.-X_{0}\right)-\left(m-\frac{1}{3}\right)^{2} t y^{3 m-2 n-1} \\
& \times\left(s-X_{2}-\frac{3 n c}{3 m-1}\right)\left(s-X_{2}+\frac{3 c}{(3 m-1) t} y^{n-m} D_{x}\right)
\end{aligned}
$$

ii) $m_{1}=2, n \geq 2 m, m \geq 2 . c=\frac{2}{3}\left(1-\frac{1}{n}\right)+\frac{m}{n}-1(\neq 0)$.

$$
\begin{aligned}
& x\left(s-X_{0}\right)-2 t y^{m-1}\left(s-X_{1}\right), \\
& y^{n-2 m+1}\left(s-X_{0}-c t y^{m-1} D_{x}\right)-\frac{2(3 m-2)}{3} t^{2} x\left(s-X_{2}\right) .
\end{aligned}
$$

a) $n+2>3 m \quad \mathfrak{a}: f=\left(x, y^{m-1}\right)$ type $y S^{\#} \quad Z_{i, p}: \begin{aligned} & n=3 i+p+4 \\ & m=i+2\end{aligned}$

$$
\left(s-X_{1}\right)\left(s-X_{2}\right)-\frac{3 y^{n-3 m+2}}{4(3 m-2) t^{3}}\left(s-X_{0}+2 c\right)\left(s-X_{0}-c t y^{m-1} D_{x}\right)
$$

b) $n+2<3 m \quad$ a: $f=\left(x, y^{n-2 m+1}\right)$ type $y X_{0}^{p}$

$$
\left(s-X_{0}+2 c\right)\left(s-X_{0}-c t y^{m-1} D_{x}\right)-4\left(m-\frac{2}{3}\right) t^{3} y^{3 m-n-2}\left(s-X_{1}\right)\left(s-X_{2}\right) .
$$

\section{§ 21. Non-isolated Singularities}

We give some examples of $b$-functions of non-isolated singularities.

Example 4. 20。 $f=x^{n}+y^{l} z^{m} . \quad d=$ g.c.d. $(l, m)$.

1. $\quad d=1$.

$$
\begin{aligned}
b(s)(s+1)\left(\prod_{i=0}^{n-2}\left(s+\frac{i+1}{n}+1\right) \prod_{\substack{0 \leq i \leq n-2 \\
0 \leq j \leq l-2}}\left(s+\frac{i+1}{n}+\frac{j+1}{l}\right)\right. \\
\left.\times \prod_{\substack{0 \leq i \leq n-2 \\
0 \leq k \leq m-2}}\left(s+\frac{i+1}{n}+\frac{k+1}{m}\right)\right)_{\text {red }} .
\end{aligned}
$$

2. $d \geq 2$. Put $l^{\prime}=l / d, m^{\prime}=m / d$ 。 


$$
\begin{aligned}
b(s) \mid(s+1) & \prod_{\substack{1 \leq t \leq d-1 \\
0 \leq i \leq n-2}}\left(s+\frac{i+1}{n}+\frac{t}{d}\right)\left(\prod_{i=0}^{n-2}\left(s+\frac{i+1}{n}+1\right)\right. \\
& \left.\times \prod_{\substack{0 \leq i \leq n-2 \\
0 \leq j \leq l-2 \\
j \neq t l^{\prime}-1}}\left(s+\frac{i+1}{n}+\frac{j+1}{l}\right)_{\substack{0 \leq i \leq n-2 \\
0 \leq k \leq m-2 \\
m \neq t m^{\prime}-1}}\left(s+\frac{i+1}{n}+\frac{k+1}{m}\right)\right)_{\mathrm{red}} .
\end{aligned}
$$

We can prove these formulae by explicit construction of differential operator $P(s)$ such that $P(s) f^{s+1}=b^{\prime}(s) f^{s}$, and the estimate in Theorem 3. 3.

Recall that the integral local monodromy of $f$ is

$$
\begin{aligned}
& H_{0}=\mathbb{Z} \quad \text { id }
\end{aligned}
$$

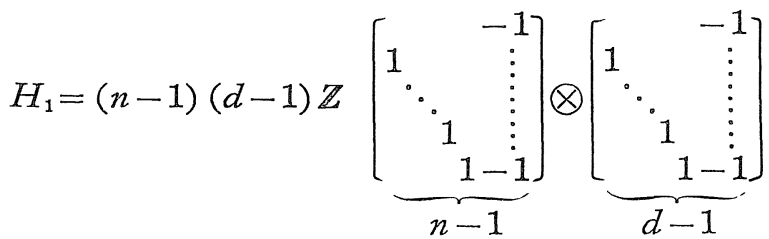

$$
\begin{aligned}
& H_{2}=(n-1) d \mathbb{Z}[\underbrace{\left[\begin{array}{ccc}
1 & & -1 \\
1 & & \vdots \\
& \ddots & \vdots \\
& 1 & \vdots \\
& 1-1
\end{array}\right]}_{n-1} \otimes \underbrace{\left[\begin{array}{lll}
1 & & \\
1 & \\
& \ddots & \\
& & 1
\end{array}\right]}_{d} .
\end{aligned}
$$

Example 4. 2 $\mathbb{1}$. $f=\sum_{i=1}^{k} x_{2 i-1} x_{2 i}^{p_{i}}$

$$
b(s)=(s+1)\left(\prod_{0 \leq i, j \leq p_{j}-1}\left(s+\frac{i_{1}+1}{p_{1}}+\cdots+\frac{i_{k}+1}{p_{k}}\right)\right)_{\mathrm{red}} .
$$

Example $\mathbb{4}_{0}$ 22: $f=\sum_{i=1}^{k}\left(x_{2 i-1} x_{2 i}\right)^{2}$

$$
b(s) \mid(s+1) \prod_{i=0}^{k}\left(s+\frac{k}{2}+\frac{i}{2}\right)^{k+1-i} .
$$

Note the equality

$$
\frac{1}{4}\left(\sum x_{2 i}^{2} D_{2 i-1}^{2}\right) f^{s+1}=(s+1)\left(s+\frac{k}{2}\right)\left(\mathbb{I} x_{2 i}^{2}\right) f^{s} .
$$

Example 4. 23. Cubic cones in $\mathbb{C}^{3}$ 
Cubic cones in $\mathbb{C}^{3}$ are classified in nine types.

1. $x^{3}+y^{3}+z^{3}-3 \lambda x y z \quad(s+1)^{2}\left(s+\frac{4}{3}\right)\left(s+\frac{5}{3}\right)\left(s+\frac{6}{3}\right)$

$$
\lambda^{3} \neq 1
$$

2. $x^{3}+y^{3}-3 x y z$

$$
(s+1)^{3}\left(s+\frac{4}{3}\right)\left(s+\frac{5}{3}\right)
$$

3. $x^{2} z-y^{3}$

$$
(s+1)\left(s+\frac{4}{3}\right)\left(s+\frac{5}{3}\right)\left(s+\frac{5}{6}\right)\left(s+\frac{7}{6}\right)
$$

4. $x^{3}+x y z$

$$
(s+1)^{3}\left(s+\frac{4}{3}\right)\left(s+\frac{5}{3}\right)
$$

5. $x^{2} z+y z^{2}$

$$
(s+1)^{2}\left(s+\frac{3}{4}\right)\left(s+\frac{5}{4}\right)
$$

6. $x y z$

$$
(s+1)^{3}
$$

7. $x^{3}+y^{3}$

$$
(s+1)^{2}\left(s+\frac{2}{3}\right)\left(s+\frac{4}{3}\right)
$$

8. $x^{2} y$

$$
(s+1)^{2}\left(s+\frac{1}{2}\right)
$$

9. $x^{3}$

$$
(s+1)\left(s+\frac{1}{3}\right)\left(s+\frac{2}{3}\right) .
$$

The polynomials in $s$ written in the righthand side are the $b$-functions of the lefthand side except 2 and 4 . In cases 2 and 4 , the factor $(s+1)^{3}$ might be $(s+1)^{2}$ for $b$-function. The diagram of specialization is as follows.

$$
\begin{aligned}
& 1 \\
& \downarrow \\
& 2 \rightarrow 4 \rightarrow 6 \\
& \downarrow \\
& \stackrel{\downarrow}{3} \rightarrow \stackrel{\downarrow}{5} \rightarrow \stackrel{\downarrow}{7} \rightarrow 8 \rightarrow 9 .
\end{aligned}
$$

It should be noted that the maximal root of $b(s)=0$ increases along the arrows.

\section{References}

[1] Arnol'd, V. I., Normal forms of functions with simple critical points, the Weyl groups $A_{h}, D_{k}, E_{k}$ and Lagrange manifolds, Functional Anal. Appl. 6 (1972), 3-25. 
[2] ___ - Normal forms of functions in neighborhoods of degenerate critical points, Russian Math. Surveys, 29 (1974), 10-50.

[3] - Classification of unimodal critical points of functions, Functional Anal. Appl., 7 (1973), 230-231.

[4] Classification of bimodal critical points of functions, Functional Anal. Appl., 9 (1975), 45-50.

[5] - Critical points of smooth function and their normal forms, Russian Math. Surveys, 30 (1975), 3-65.

[6] Bernstein, I. N., Modules over the ring of differential operators. Study of fundamental solution of differential equations with constant coefficients, Functional Anal. Appl., 2 (1971). 1-16.

[7] The analytic continuation of generalized functions with respect to a parameter, Functional Anal. Appl., 6 (1972), 26-40.

[8] Björk, J. K., On the dimension of the modules over the ring of differential operators, to appear.

[9] Hironaka, H., Resolutions of singularities of algebraic varieties over the field of characteristic zero, Ann. of Math., 79 (1964), 109-326.

[10] Kashiwara, M. and Kawai, T., Microlocal properties of $\prod_{j=1}^{s} f_{j^{+}}^{s_{j}}$, Proc. Japan Acad., 51 (1975), 270-272.

[11] Kashiwara, M., Algebraic study of differential equations, Master thesis Univ. Tokyo, 1967 (in Japanese).

[12] $\quad b$-functions and the singularity of hypersurface, RIMS Kokyuroku, 225 (1975), 16-53. (in Japanese).

[13] - On the maximally overdetermined system of linear differential equations, I. Publ. RIMS, Kyoto Univ., 10 (1975), 563-579.

[14] _- On the rationality of the roots of $b$-functions, to appear.

[15] Malgrange, B., Intégral asymptotiques et monodromie, Ann. Sci. Ecole Norm. Sup., 7 (1974), 405-430.

[16] _ _ On the polynomial of I. N. Bernstein, Russian Math. Surveys, 29 (1974), $81-88$.

[17] - Sur les polynomes de I. N. Bernstein, to appear.

[18] ___ Polynomes de Bernstein pour les singularite isoleé, to appear.

[19] Miwa, T., Determination of $b(s)$-the case of quasi-homogeneous isolated singularity, RIMS Kokyuroku, Kyoto Univ., 225 (1975), 62-71. (in Japanese).

[20] Saito, K., Einfach-elliptische Singularitäten. Invent. Math., 23 (1974), 289-325.

[21] — - On the uniformization of complements of discrimiant loci, A. M. S. Summer Institute 1975.

[22] _ _ Q Quasihomogene isolierte singularitaten von Hyperflachen, Invent. Math., 14 (1971), 123-142.

[23] Sato, M., Theory of prehomogeneous vector spaces, (noted by T. Shintani), Sugaku no Ayumi, 15-1, 1970 (in Japancse).

[24] - Reduced b-functions, (Noted by T. Yano), RIMS Kokyuroku, Kyoto Univ., 225 (1975), (in Japanese).

[25] Sato, M., Kawai, T. and Kashiwara, M., Microfunctions and pseudo-differential equations, Proceeding of Katata Conf., Lecture Notes in Math., 287, Springer, (1973), 263-529.

[26] Sato, M. and Shintani, T., On zeta functions associated with prehomogeneous vector space, Ann. of Math., 100 (1974), 131-170.

[27] Yano, T., The theory of $b$-functions, Master thesis of Kyoto Univ., 1974. (in Japanese).

[28] , The theory of $b$-functions. RIMS Kokyuroku, Kyoto Univ., 225 (1975), 72-234. (in Japanese). 
[29] - Some progresses in b-function theory, RIMS Kokyuroku, Kyoto Univ., 248 (1975), 293-311. (in Japanese).

[30] , Some topics in b-function theory, RIMS Kokyuroku, Kyoto Univ., 266 (1976), 319-329. (in Japanese).

[31] $\longrightarrow$ The theory of $b$-functions, to appear in Sugaku, (in Japanese).

[32] - On the theory of $b$-functions II, in preparation.

[33] - On the microlocal structure of weighted homogeneous polynomials associated to Coxeter groups, in preparation.

[34] - On (sub-) holonomicity, to appear in RIMS Kokyuroku, Kyoto Univ., 287 (1977), 10-41.

[35] Yano, T. and Sekiguchi, J., On the discriminant of Coxeter groups, in preparation.

[36] - On the microlocal structure of weighed homogeneous polynomials associated to Coxeter groups, with some Lie algebra related to GL(2), to appear in RIMS Kokyuroku, Kyoto Univ., 1976. (in Japanese).

[37] Yano, T., On the holonomic system of $f^{s}$ and $b$-function, Publ. RIMS, Kyoto Univ., 12, Supplement, (1977), in press.

[38] Atiyah, M. F., Resolution of singularities and division of distributions, Comm. Pure Appl. Math., 23 (1970), 145-150.

[39] Bernstein, I. N. and Gel'fand, S. I., Meromorphy of the function $P^{\lambda}$, Functional Anal. Appl., 3 (1969), 84-86.

[40] Arnold, V. I., Local normal forms of functions, Invent. Math., 35 (1976), 87-109.

[41] Kashiwara, M., Kimura, T. and Muro, M., On the microlocal calculus and its application to simple holonomic systems, to appear. 